\title{
DYNAMICS OF AXIALLY SYMMETRIC PERTURBED HAMILTONIANS IN 1:1:1 RESONANCE
}

\author{
D. CARRASCO, J.F. PALACIÁN, C. VIDAL, J. VIDARTE, AND P. YANGUAS
}

\begin{abstract}
We study the dynamics of a family of perturbed three-degreesof-freedom (3-DOF) Hamiltonian systems which are in 1:1:1 resonance. The perturbation consists of axially symmetric cubic and quartic arbitrary polynomials. Our analysis is performed by normalisation, reduction and KAM techniques. Firstly, the system is reduced by the axial symmetry and then, periodic solutions and KAM 3-tori of the full system are determined from the relative equilibria. Next, the oscillator symmetry is extended by normalisation up to terms of degree 4 in rectangular coordinates; after truncation of higher orders and reduction to the orbit space, some relative equilibria are established and periodic solutions and KAM 3-tori of the original system are obtained. As a third step, the reduction of the two symmetries leads to a one-degrees-offreedom system that is completely analysed in the twice reduced space. All the relative equilibria, together with the stability and parametric bifurcations are determined. Moreover the invariant 2-tori (related to the critical points of the twice reduced space), some periodic solutions and the KAM 3-tori, all corresponding to the full system, are established. Additionally, the bifurcations of equilibria occurring in the twice reduce space are reconstructed as quasi-periodic bifurcations involving 2-tori and periodic solutions of the full system.
\end{abstract}

2010 Mathematics Subject Classification. Primary 34C15, Secondary 34C20, 34C23, 34C25. Key words and phrases. Invariants; symplectic reductions; axial symmetry; relative equilibria; periodic solutions; parametric bifurcations; invariant tori; reconstruction of the flow.

The authors are partially supported by Projects MTM 2011-28227-C02-01 of the Ministry of Science and Innovation of Spain, MTM 2014-59433-C2-1-P of the Ministry of Economy and Competitiveness of Spain, MTM2017-88137-C2-1-P of the Ministry of Economy, Industry and Competitiveness of Spain and by Project DIUBB 165708 3/R, Universidad del Bío-Bío, Chile. This paper is part of Jhon Vidarte's Ph.D. Thesis in the Program "Doctorado en Matemática Aplicada, Universidad del Bío-Bío". 


\section{INTRODUCTION}

The study of perturbations of isotropic harmonic oscillators is a classical subject. We can find in the literature pure mathematical works, as well as applications in many fields. Perturbed harmonic oscillators in 1:1:1 resonance model, for instance, the near-harmonic motion in the core of an elliptical galaxy $[75,12,72,73,74,8$, $4,9]$. The existence of stars whose orbits lie out of the main plane of the galaxy cannot be explained by the perturbations of a planar dynamical system. Thus, one should consider the 3-DOF case, the so called triaxial elliptical galaxies [72]. The situation is similar in other fields in physics and chemistry, such as molecular dynamics. Indeed, small-amplitude vibrations in molecules follow an oscillatory law, as their spectra and their reactions show [36]. Chemists adopt models based on perturbations of harmonic oscillators to describe the motion of the nuclei in small molecules [17, 24]. The dynamics of ions in Penning traps [44] can be also studied in this setting. More applications are found in mechanical engineering, wave mechanics, semiclassical quantization, lattices, non-linear optics, or statistical mechanics. For references see, for instance, [47] and references therein.

We tackle the analysis of our 3-DOF problem under the light of normal forms [18, $54]$ and singular reduction $[2,15]$. In [57] we can find a geometric interpretation of singular reduction theory in the setting of resonant Hamiltonians with $n$ degrees of freedom. A generalisation is in progress [58]. We apply some of the results of [57] in the present problem. Our starting Hamiltonian system can be reduced both by one exact symmetry and by one approximate symmetry. On the one hand, the system is axially symmetric, but on the other hand, the oscillator symmetry may be extended by normalisation up to a certain order with respect to a small parameter. So, we can reduce the system (i) by the axial symmetry, (ii) by the oscillator symmetry (after truncation) or (iii) by both symmetries. Each reduced system provides information about the original one. In this paper we analyse the three reduced systems in their corresponding reduced spaces and perform the reconstruction of the flow corresponding to the starting Hamiltonian in the three situations. 
The coordinates we use to perform the analysis of the reduced space are the so called invariants. They are quantities intrinsic to the symmetry that is reduced and are used to parametrise the reduced space, thus they are global coordinates of the reduced space.

For the reconstruction of invariant 3-tori we apply the KAM-type theorem by Han, Li and Yi [39], that can be applied to highly degenerate systems as ours is, and allows us to get some families of invariant tori of dimension three for the starting Hamiltonian. These 3-tori are located around the periodic solutions that we establish in cases (i) and (ii) and around the periodic solutions and invariant 2tori that we obtain in case (iii). The characterisation of these KAM tori by applying Han-Li-Yi's Theorem is new compared to the paper [31].

Along the paper we will see that certain types of periodic solutions and invariant 2-tori are obtained as relative equilibria of the vector fields related to the reduced Hamiltonian systems in the three different situations. More specifically, solutions whose projection in configuration space $O x y z$ are rectilinear trajectories in the $O z$ axis (from now on, rectilinear trajectories in the $O z$-axis) are studied in the spaces resulting after reducing the symmetries (ii) and (iii). Periodic solutions in $T^{*} \mathbb{R}^{3}$ whose projections in configuration space are close to circular solutions on the $O x y$ plane (from now on, circular-equatorial solutions) are analysed using the reductions related to the cases (i) and (ii). Finally, the invariant 2-tori are determined in the reduced space that results after applying both symmetries, that is, case (iii). Hence, different reductions provide us with different features of the original system.

Perturbations of the 1:1:1 resonance have been previously studied under the point of view of singular reduction. In [16], the bases for the reduction of axially symmetric perturbations of the isotropic harmonic oscillator are established. As an example, the authors apply their results to a particular case of an axially symmetric quartic perturbation. Later on, Ferrer et al [34] consider the normalisation and reduction of perturbations that are arbitrary cubic and quartic polynomials. The integrability of certain subfamilies is also analysed. Paper [31] treats the reduction 
process, analysis of the reduced system and reconstruction of axially symmetric cubic potentials. Although the perturbation is only at first order, a second order in the normalisation procedure is required to obtain a non-trivial normal form.

Thus, as it is pointed out in the introduction of [31], rigorously, the second order perturbation (i.e., the quartic terms) should be taken into account in the formulation of the starting system. In this sense, our study comes to complete the work started in [31], as the perturbation considered here is composed by axiallysymmetric cubic and quartic homogeneous polynomials.

As we will see throughout the paper, the main difference between taking into account only cubic perturbations and taking both cubic and quartic ones is that, in the first case the dynamics in the twice reduced space depends only on two essential parameters, whereas in the second case it depends on three. Consequently, the consideration of cubic and quartic terms leads to a more complex analysis. The bifurcation lines in [31] are now bifurcation surfaces. We recover the same type of bifurcations that appeared in [31] and find a new one, a $\mathbb{Z}$-symmetric $A_{5}^{+}$(see a description of it in [40]), also called a butterfly $A_{5}$ with reflection symmetry [64].

Moreover, in this paper we resort to recent KAM results, as the one by Han, $\mathrm{Li}$ and $\mathrm{Yi}$ [39], for the explicit reconstruction of certain KAM 3-tori. These KAM tori appear as Cantor-like families of Diophantine invariant tori that depend on the external parameters of the problem (five in our case) as well as three internal parameters (three due to the dimension of the 3-tori), see [6].

Also, apart from establishing the existence of families of periodic solutions and invariant 3-tori from the twice reduced system, we determine periodic solutions and tori from the systems that are reduced either by the oscillator symmetry or by the axial symmetry. The analysis of the periodic solutions combines techniques of averaging and reduction theories [60,11], and in particular is based on Reeb's Theorem [62] for getting families of periodic solutions from the critical points of a reduced system defined on the reduced space. Additional results and some more 
theory on this topic have been developed in [71], see also [54, 55, 57], and we shall make use of them along the paper.

In [41] and [42], axially-symmetric perturbed isotropic harmonic oscillators are considered under the point of view of bifurcation theory, the perturbation being a cubic polynomial. In particular, Hanßmann and van der Meer have focused on the Hamiltonian Hopf bifurcation occurring in this context, devising methods to determine the presence of this type of bifurcation in three-degrees-of-freedom systems by putting the bifurcation into standard form, see also [40]. We shall follow their procedure in Section 5, but with an appropriate set of rectangular coordinates that are introduced in Section 4. The monograph [69] contains a detailed analysis of the second non-trivial normal form corresponding to the potential handled in [31], focusing on the occurrence of different types of Hamiltonian Hopf bifurcations and a global bifurcation that cannot be analysed if the normalisation is carried out only up to terms of order 2 in $\epsilon$.

In a series of papers, Efstathiou and collaborators analyse perturbations of the 1:1:1 resonance. In [23] they consider point-group-invariant perturbations and calculate stationary points that can undergo a linear Hamiltonian Hopf bifurcation, see also the monograph [21]. In [22] a cubic potential with tetrahedral symmetry is chosen and, after normalisation and reduction, they determine relative equilibria and bifurcations. As well as Hanßmann and van der Meer these authors also use symplectic reduction and invariant theory combined with the introduction of variables of symplectic nature to deal with the stability character of the periodic solutions. The sets of variables built in these papers usually lead to very long expressions compared with the coordinates we introduce in Sections 3, 4 and 5 for similar purposes. In this respect it is expected that the various bifurcation analyses performed in these papers, as well as other related studies on bifurcations, KAM theory, and so on can be better accomplished using the sets of symplectic coordinates we build to deal with the analysis of the relative equilibria in the various reduced spaces appearing in the paper. 
The type of potentials we tackle in this paper has been considered as well by some authors with the aim of studying the existence of periodic solutions, see for example the works of Llibre and coworkers $[48,49,7]$. Also, the potential considered in [35] is a particular case of ours. Applying averaging theory these authors find families of periodic solutions and in some cases study their linear stability. Other papers dealing with the 1:1:1 resonance in the framework of the analysis of periodic solutions are $[75,72,73,8]$ and more recently $[4,9,46]$. Traditionally, averaging is applied by means of different sets of coordinates. As they do not use the invariants of the corresponding reduced spaces, their treatment can lack on the generality to cover the whole reduced space. An exception is [61] where a potential without axial symmetry is analysed in the reduced space using the associated invariants of the reduction together with some convenient sets of local symplectic coordinates that were introduced for each type of motion we deal with.

Resonances in Hamiltonian systems with three or more degrees of freedom have been received also the attention of many authors from the point of view of the determination of periodic solutions and their possible bifurcations, reduction theory, existence of chaos and other related topics, see for instance $[1,43,38,37,27,63]$. In this case the dimension of the orbit space is at least four and the theory turns out rather cumbersome. Extensions to the 1:1:1:1 resonance have been considered by Egea et al $[25,26]$. There the authors use reduction and invariant theory, achieving the existence of families of invariant tori of different dimensions (starting with periodic solutions) as well as their parametric bifurcations. In these studies additional symmetries are attached to the perturbation with the aim of exploring the relationship between the 1:1:1:1 resonance and the spatial two-body problem by means of the Kustaanheimo-Stiefel regularisation technique.

The paper is divided into eight sections. In Section 2 we introduce the Hamiltonian function while in Section 3 we apply the reduction by the axial symmetry and perform the reconstruction to the starting (or full) Hamiltonian system. 
The reduction is singular and the reduced space presents a singularity that corresponds, precisely, to the rectilinear periodic trajectories in the $O z$-axis. The vector field related to the reduced Hamiltonian is analysed as a function of the invariants associated to the axial symmetry. On the one hand, there are two relative equilibria that correspond to linearly stable prograde and retrograde nearly circular-equatorial periodic trajectories in the full system. On the other hand, the existence of 3-dimensional KAM tori around these solutions is proved under certain non-degeneracy conditions.

Section 4 accounts for the reduction of the oscillator symmetry. The starting Hamiltonian function is normalised up to including polynomials of degree four and the truncated normalised (and reduced) system is analysed in the corresponding reduced space, which is $\mathbb{C P}_{h}^{2}$. In this case the reduction is regular and we find again the two relative equilibria that correspond to the prograde and retrograde nearly circular periodic trajectories on the $O x y$-plane and another equilibrium that is associated to the rectilinear solutions in the $O z$-axis. The conditions for the existence of the corresponding periodic solutions and KAM 3-tori are explicitly calculated.

Section 5 is devoted to the analysis of the twice reduced system. A complete study of the relative equilibria and parametric bifurcations is presented. The reconstruction of the corresponding invariant 2-tori (and periodic solutions) is given in Section 6. Moreover, the parametric bifurcations occurring between the relative equilibria in the twice reduced space are also reconstructed for the full system, leading to a rather complete description of the Hamiltonian system in 1:1:1 resonance with an axially symmetric perturbation composed of polynomials of degrees three and four. In addition to this, the persistence of KAM 3-tori related to the elliptic (non-degenerate) points is also proved using Han-Li-Yi's Theorem.

In Section 7 we treat the case of perturbations that are not axially symmetric but such that the combinations between the parameters make the system to enjoy the axial symmetry up to terms of degree four. Then, one can reduce the Hamiltonian 
two times and use the theory developed in previous sections. In this situation some consequences for the starting system can be drawn using the same reconstruction procedures as in the axially symmetric context.

The paper ends with a section devoted to the conclusions and future work.

\section{Axially symmetric Perturbed Hamiltonian}

Consider the autonomous 3-DOF Hamiltonian system

$$
v^{\prime}=\mathbb{J} \nabla \mathcal{H}(v), \quad v=(q, p),
$$

with $\mathbb{J}$ denoting the standard $6 \times 6$ skew symmetric matrix and $\mathcal{H}(v)$ as the Hamiltonian function

$$
\mathcal{H}(v)=\mathcal{H}_{0}(v)+\mathcal{H}_{1}(q)+\mathcal{H}_{2}(q)
$$

Hamiltonian $\mathcal{H}_{0}$ corresponds to the isotropic harmonic oscillator

$$
\mathcal{H}_{0}(v)=\frac{1}{2}\left(x^{2}+X^{2}\right)+\frac{1}{2}\left(y^{2}+Y^{2}\right)+\frac{1}{2}\left(z^{2}+Z^{2}\right),
$$

whereas $\mathcal{H}_{1}$ and $\mathcal{H}_{2}$ are the general homogeneous polynomials of degrees three and four, respectively, in the coordinates $q=(x, y, z)$, i.e.,

$$
\begin{aligned}
\mathcal{H}_{1}(q)= & a_{1} x^{3}+a_{2} x^{2} y+a_{3} x y^{2}+a_{4} y^{3}+a_{5} x^{2} z+a_{6} x y z+a_{7} y^{2} z+a_{8} x z^{2} \\
& +a_{9} y z^{2}+a_{10} z^{3}, \\
\mathcal{H}_{2}(q)= & b_{1} x^{4}+b_{2} x^{3} y+b_{3} x^{2} y^{2}+b_{4} x y^{3}+b_{5} y^{4}+b_{6} x^{3} z+b_{7} x^{2} y z+b_{8} x y^{2} z \\
& +b_{9} y^{3} z+b_{10} x^{2} z^{2}+b_{11} x y z^{2}+b_{12} y^{2} z^{2}+b_{13} x z^{3}+b_{14} y z^{3}+b_{15} z^{4},
\end{aligned}
$$

with $a_{j}$ and $b_{j}$ real parameters.

We focus our attention on Hamiltonian functions that are invariant under the axial $S^{1}$-action

$$
\begin{array}{rlc}
\varrho: \quad S^{1} \times T^{*} \mathbb{R}^{3} & \rightarrow & T^{*} \mathbb{R}^{3} \\
(\nu,(q, p)) & \mapsto \quad\left(\exp _{\nu} q, \exp _{\nu} p\right)
\end{array}
$$


where $T^{*} \mathbb{R}^{3} \cong \mathbb{R}^{3} \times \mathbb{R}^{3}$ and $\exp _{v}$ stands for the rotation

$$
\exp _{\nu}=\left(\begin{array}{ccc}
\cos \nu & -\sin \nu & 0 \\
\sin \nu & \cos \nu & 0 \\
0 & 0 & 1
\end{array}\right)
$$

The following result characterises the family of Hamiltonian functions of the form (1) that are invariant under rotations with respect to the $S^{1}$-action $\varrho$.

Proposition 2.1. Hamiltonian (1) is axially symmetric with respect to the Oz-axis when the functions $\mathcal{H}_{1}$ and $\mathcal{H}_{2}$ are of the form

$$
\begin{aligned}
& \mathcal{H}_{1}(q)=a_{5} z\left(x^{2}+y^{2}\right)+a_{10} z^{3} \\
& \mathcal{H}_{2}(q)=b_{1}\left(x^{2}+y^{2}\right)^{2}+b_{10} z^{2}\left(x^{2}+y^{2}\right)+b_{15} z^{4}
\end{aligned}
$$

Proof We denote by $N=x Y-y X$ the third component of the angular momentum vector. The condition for $\mathcal{H}$ in (1) to be invariant under rotations around the $O z$ axis is $\{\mathcal{H}, N\}=0$, where $\{$, $\}$ designates the usual Poisson bracket operator. Imposing that $\mathcal{H}$ commutes with $N$ we arrive at an equation where the following conditions have to be satisfied, namely, $a_{1}=a_{2}=a_{3}=a_{4}=a_{6}=a_{8}=a_{9}=0$, $a_{7}=a_{5}, b_{2}=b_{4}=b_{6}=b_{7}=b_{8}=b_{9}=b_{11}=b_{13}=b_{14}=0, b_{3}=2 b_{1}, b_{12}=b_{10}$, $b_{5}=b_{1}$. Thus, we end up with Hamiltonian (5) and conclude that $N$ is an integral of motion for it.

Let us introduce $\mathcal{H}_{\epsilon}$ as a small perturbation of $\mathcal{H}_{0}$. More specifically, the Hamiltonian function tackled in this paper is

$$
\mathcal{H}_{\epsilon}(q, p)=\mathcal{H}_{0}(q, p)+\epsilon \mathcal{H}_{1}(q)+\epsilon^{2} \mathcal{H}_{2}(q)
$$

with $\mathcal{H}_{1}$ and $\mathcal{H}_{2}$ given explicitly in (5). The dimensionless parameter $\epsilon$ expresses the fact that we consider the cubic and quartic perturbations to be small. Indeed, $\epsilon$ can always be introduced by a scaling $(q, p) \rightarrow \epsilon(q, p)$ with the corresponding time scaling, so that the function $\mathcal{H}$ in (1) takes the form (6). Thus, $\mathcal{H}_{\epsilon}$ given in (6) describes the dynamics of the Hamiltonian system close to the origin. 


\section{REDUCTION BY THE AXIAL SYMMETRY AND RECONSTRUCTION}

3.1. Reduction by the axial symmetry. As we have seen in the previous section, Hamiltonian (6) is invariant under the $S^{1}$-action $\varrho$. This action is not free because it has non-trivial isotropy groups. In fact, the subspace $\{(0,0, z, 0,0, Z) \mid z, Z \in \mathbb{R}\}$ is invariant and consequently, the reduction by the axial symmetry is singular, see details in $[16,31]$.

We apply this symmetry to simplify the analysis of the Hamiltonian system associated to (6) by lowering the number of degrees of freedom from three to two.

We introduce the invariants associated to this symmetry, namely,

$$
\begin{array}{lll}
i_{1}=x^{2}+y^{2}, & i_{2}=X^{2}+Y^{2}, & i_{3}=x Y-y X=N, \\
i_{4}=x X+y Y, & i_{5}=z, & i_{6}=Z .
\end{array}
$$

They satisfy the constraints

$$
i_{1} i_{2}=i_{3}^{2}+i_{4}^{2}, \quad i_{1} \geq 0, \quad i_{2} \geq 0
$$

see details in $[16,31,55]$. The invariants $i_{1}, \ldots, i_{6}$ subject to the constraints $(8)$ uniquely specify an orbit. The Poisson brackets among the invariants are given in Table 1. Notice that Proposition 2.1 can be also directly inferred from (7).

\begin{tabular}{|c||c|c|c|c|c|c|}
\hline$\left\{i_{j}, i_{k}\right\}$ & $i_{1}$ & $i_{2}$ & $i_{3}$ & $i_{4}$ & $i_{5}$ & $i_{6}$ \\
\hline \hline$i_{1}$ & 0 & $4 i_{4}$ & 0 & $2 i_{1}$ & 0 & 0 \\
\hline$i_{2}$ & $-4 i_{4}$ & 0 & 0 & $-2 i_{2}$ & 0 & 0 \\
\hline$i_{3}$ & 0 & 0 & 0 & 0 & 0 & 0 \\
\hline$i_{4}$ & $-2 i_{1}$ & $2 i_{2}$ & 0 & 0 & 0 & 0 \\
\hline$i_{5}$ & 0 & 0 & 0 & 0 & 0 & 1 \\
\hline$i_{6}$ & 0 & 0 & 0 & 0 & -1 & 0 \\
\hline
\end{tabular}

TABLE 1. Poisson brackets for the invariants $i_{j}$.

After fixing $i_{3}=N=\gamma$, the reduced space is the non-compact 4-dimensional orbifold

$$
\mathcal{M}_{\gamma}=\left\{\left(i_{1}, i_{2}, i_{4}, i_{5}, i_{6}\right) \in \mathbb{R}^{5} \mid i_{1} i_{2}-i_{4}^{2}=\gamma^{2}, i_{1} \geq 0, \quad i_{2} \geq 0\right\} .
$$


The space is regular (a symplectic manifold) provided $\gamma \neq 0$. When $\gamma=0$ the reduced space $\mathcal{M}_{0}$ is singular. The motions related to any point in $\mathcal{M}_{0}$ are the ones whose projections onto $O x y z$-space are perpendicular to the $O x y$-plane, that is, trajectories of polar type. In particular, all rectilinear solutions passing through the origin of $\mathbb{R}^{6}$ are contained in $\mathcal{M}_{0}$. The space $\mathcal{M}_{0}$ has a 2-dimensional singular subspace given by points of the form $\left(0,0,0, i_{5}, i_{6}\right)$ with $i_{5}, i_{6}$ in $\mathbb{R}$; these correspond to the rectilinear solutions occurring on the $O z Z$-plane. See also $[16,55]$.

Hamiltonian (6) is written as a function of the invariants in the space $\mathcal{M}_{\gamma}$ through

$$
\mathcal{H}_{\epsilon}=\mathcal{H}_{\epsilon}\left(i_{1}, i_{2}, i_{4}, i_{5}, i_{6}\right)=\mathcal{H}_{0}+\epsilon \mathcal{H}_{1}+\epsilon^{2} \mathcal{H}_{2},
$$

where

$$
\begin{aligned}
& \mathcal{H}_{0}=\frac{1}{2}\left(i_{1}+i_{2}+i_{5}^{2}+i_{6}^{2}\right), \\
& \mathcal{H}_{1}=i_{5}\left(a_{5} i_{1}+a_{10} i_{5}^{2}\right), \\
& \mathcal{H}_{2}=b_{1} i_{1}^{2}+b_{10} i_{1} i_{5}^{2}+b_{15} i_{5}^{4} .
\end{aligned}
$$

The associated vector field is calculated by means of Liouville's Theorem and yields that

$$
i_{j}^{\prime}=\frac{d}{d t} i_{j}=\left\{i_{j}, \mathcal{H}_{\epsilon}\right\}=\sum_{\substack{k=1 \\ k \neq 3}}^{6}\left\{i_{j}, i_{k}\right\} \frac{\partial \mathcal{H}_{\epsilon}}{\partial i_{k}}, \quad j \in\{1,2,4,5,6\},
$$

where

$$
\begin{aligned}
& i_{1}^{\prime}=2 i_{4}, \\
& i_{2}^{\prime}=-2 i_{4}\left[1+2 \epsilon a_{5} i_{5}+2 \epsilon^{2}\left(2 b_{1} i_{1}+b_{10} i_{5}^{2}\right)\right], \\
& i_{4}^{\prime}=i_{2}-i_{1}-2 \epsilon a_{5} i_{1} i_{5}-2 \epsilon^{2} i_{1}\left(2 b_{1} i_{1}+b_{10} i_{5}^{2}\right), \\
& i_{5}^{\prime}=i_{6}, \\
& i_{6}^{\prime}=-i_{5}-\epsilon\left(a_{5} i_{1}+3 a_{10} i_{5}^{2}\right)-2 \epsilon^{2} i_{5}\left(b_{10} i_{1}+2 b_{15} i_{5}^{2}\right) .
\end{aligned}
$$


The zeroes of system (13) are related to periodic solutions of the Hamiltonian $\mathcal{H}_{\epsilon}$. As we consider $\epsilon$ to be small, we will determine only the equilibria that correspond to periodic solutions that are close to the origin. In case we would not assume $\epsilon$ small we could find other relative equilibria.

3.2. Periodic solutions from $\mathcal{M}_{\gamma}$. There are two types of periodic solutions for the system defined by (6) that can be studied in $\mathcal{M}_{\gamma}$, namely, the ones coming from the relative equilibria of (13) and the solutions whose projection in configuration space $O x y z$ are near rectilinear trajectories in the $O z$-axis. These rectilinear motions appear in the singular space $\mathcal{M}_{0}$.

Proposition 3.1. Assume $\gamma \neq 0$ and $\epsilon$ sufficiently small. Then the vector field (13) has two isolated equilibria on the reduced space $\mathcal{M}_{\gamma}$, namely, the point $\mathcal{O}^{1}$ with coordinates

$$
\begin{aligned}
& i_{1}^{1}=-\gamma+\epsilon^{2}\left(a_{5}^{2}-2 b_{1}\right) \gamma^{2}+O\left(\epsilon^{4}\right), \\
& i_{2}^{1}=-\gamma-\epsilon^{2}\left(a_{5}^{2}-2 b_{1}\right) \gamma^{2}+O\left(\epsilon^{4}\right), \\
& i_{4}^{1}=0, \\
& i_{5}^{1}=\epsilon a_{5} \gamma-\epsilon^{3} a_{5}\left(a_{5}^{2}+3 a_{5} a_{10}-2 b_{1}-2 b_{10}\right) \gamma^{2}+O\left(\epsilon^{4}\right), \\
& i_{6}^{1}=0,
\end{aligned}
$$

for $\gamma<0$ (retrograde motion), and the point $\mathcal{O}^{2}$ with coordinates

$$
\begin{aligned}
& i_{1}^{2}=\gamma+\epsilon^{2}\left(a_{5}^{2}-2 b_{1}\right) \gamma^{2}+O\left(\epsilon^{4}\right), \\
& i_{2}^{2}=\gamma-\epsilon^{2}\left(a_{5}^{2}-2 b_{1}\right) \gamma^{2}+O\left(\epsilon^{4}\right), \\
& i_{4}^{2}=0, \\
& i_{5}^{2}=-\epsilon a_{5} \gamma-\epsilon^{3} a_{5}\left(a_{5}^{2}+3 a_{5} a_{10}-2 b_{1}-2 b_{10}\right) \gamma^{2}+O\left(\epsilon^{4}\right), \\
& i_{6}^{2}=0,
\end{aligned}
$$

for $\gamma>0$ (prograde motion). 
Proof The equilibrium points of system (13) on the space $\mathcal{M}_{\gamma}$ are of the form $\mathcal{O}^{*}=\left(i_{1}^{*}, i_{2}^{*}, 0, i_{5}^{*}, 0\right)$, where $i_{1}^{*}, i_{2}^{*}, i_{5}^{*}$ are the zeroes of the algebraic system

$$
\begin{aligned}
& g_{1}\left(i_{1}, i_{2}, i_{5} ; \epsilon\right)=i_{2}-i_{1}-2 \epsilon a_{5} i_{1} i_{5}-2 \epsilon^{2} i_{1}\left(2 b_{1} i_{1}+b_{10} i_{5}^{2}\right), \\
& g_{2}\left(i_{1}, i_{2}, i_{5} ; \epsilon\right)=-i_{5}-\epsilon\left(a_{5} i_{1}+3 a_{10} i_{5}^{2}\right)-2 \epsilon^{2} i_{5}\left(b_{10} i_{1}+2 b_{15} i_{5}^{2}\right), \\
& g_{3}\left(i_{1}, i_{2}, i_{5} ; \epsilon\right)=i_{1} i_{2}-\gamma^{2} .
\end{aligned}
$$

When $\epsilon=0$ the only solutions of $(14)$ are $\left(i_{1}^{*}, i_{2}^{*}, i_{5}^{*}\right)=(\mp \gamma, \mp \gamma, 0)$. It is clear that $g=\left(g_{1}, g_{2}, g_{3}\right)$ satisfies

$$
g(\mp \gamma, \mp \gamma, 0 ; 0)=(0,0,0), \quad \operatorname{det} D g(\mp \gamma, \mp \gamma, 0 ; 0)= \pm 2 \gamma .
$$

Therefore, by the implicit function theorem there exists a unique solution $\left(i_{1}(\epsilon)\right.$, $\left.i_{2}(\epsilon), i_{5}(\epsilon)\right)$ of system (14) satisfying $\left(i_{1}(0), i_{2}(0), i_{5}(0)\right)=(\mp \gamma, \mp \gamma, 0)$. From now on, we adopt the convention that when the signs “ \pm " or “干" appear in an expression involving $\gamma$, the upper sign applies for $\gamma<0$ (retrograde motions) and the lower sign applies for $\gamma>0$ (prograde motions).

To get the solutions we formally solve system (14) by proposing a Taylor expansion in terms of $\epsilon$ as

$$
i_{j}^{*}=i_{j 0}^{*}+\epsilon i_{j 1}^{*}+\epsilon^{2} i_{j 2}^{*}+\epsilon^{3} i_{j 3}^{*}+O\left(\epsilon^{4}\right), \quad j=1,2,5
$$

This leads to the conclusion of the proposition. The corresponding solutions are close to the $O x y$-plane because $i_{5}^{*}$ is nearly zero and they are nearly circular ones because one has that $\mathcal{H}_{0}$ is close to $\mp \gamma$.

Theorem 3.1. When $\gamma \neq 0$ and $\epsilon>0$ is sufficiently small, Hamiltonian (6) has periodic solutions in $T^{*} \mathbb{R}^{3}$ parametrised by $\gamma$ whose projections in configuration space are close to retrograde $(\gamma<0)$ and prograde $(\gamma>0)$ circular trajectories on the Oxy-plane. These periodic solutions are linearly stable and their periods are $2 \pi\left[1 \mp \epsilon^{2}\left|a_{5}^{2}-2 b_{1}\right| \gamma\right]+O\left(\epsilon^{4}\right)$. 
Proof Firstly, we build symplectic coordinates $\left(Q_{1}, Q_{2}, P_{1}, P_{2}\right)$ in $\mathcal{M}_{\gamma}$ as functions of the invariants given in (7). More precisely, we introduce functions $f_{1}=$ $f_{1}\left(i_{1}, i_{2}, i_{4}\right)$ and $f_{2}=f_{2}\left(i_{1}, i_{2}, i_{4}\right)$ to be determined such that the transformation

$$
Q_{1}=i_{5}, \quad P_{1}=i_{6}, \quad Q_{2}=f_{1}, \quad P_{2}=f_{2}
$$

be symplectic. Taking into account relations (7) and the Poisson brackets among the $i_{j}$ given in Table 1 , we trivially have that $\left\{Q_{1}, P_{1}\right\}=1$ and

$$
\begin{aligned}
\left\{Q_{2}, P_{2}\right\}=2[ & \frac{\partial f_{1}}{\partial i_{4}}\left(i_{2} \frac{\partial f_{2}}{\partial i_{2}}-i_{1} \frac{\partial f_{2}}{\partial i_{1}}\right)+\frac{\partial f_{2}}{\partial i_{4}}\left(i_{1} \frac{\partial f_{1}}{\partial i_{1}}-i_{2} \frac{\partial f_{1}}{\partial i_{2}}\right) \\
& \left.+2 i_{4}\left(\frac{\partial f_{1}}{\partial i_{1}} \frac{\partial f_{2}}{\partial i_{2}}-\frac{\partial f_{1}}{\partial i_{2}} \frac{\partial f_{2}}{\partial i_{1}}\right)\right] .
\end{aligned}
$$

Note also that $\left\{Q_{1}, Q_{2}\right\}=\left\{Q_{1}, P_{2}\right\}=\left\{Q_{2}, P_{1}\right\}=\left\{P_{1}, P_{2}\right\}=0$.

In order to solve the partial differential equation $\left\{Q_{2}, P_{2}\right\}=1$, we select a convenient function for $f_{1}=i_{1}$ and solve for $f_{2}$, getting

$$
f_{2}\left(i_{1}, i_{2}, i_{4}\right)=\frac{i_{4}}{2 i_{1}}
$$

Thus, the symplectic coordinates are

$$
Q_{1}=i_{5}, \quad Q_{2}=i_{1}, \quad P_{1}=i_{6}, \quad P_{2}=\frac{i_{4}}{2 i_{1}} .
$$

These coordinates are properly defined in $\mathcal{M}_{\gamma}$, except for $i_{1}=0$. In particular, they are valid in neighbourhoods of the points $\mathcal{O}^{1}$ and $\mathcal{O}^{2}$.

Using the transformation (16), we obtain the expressions of the equilibria $\mathcal{O}^{1}$ and $\mathcal{O}^{2}$ in the coordinates $(Q, P)$. Specifically they are $\left(Q_{1}^{*}, Q_{2}^{*}, P_{1}^{*}, P_{2}^{*}\right)$, with

$$
\begin{aligned}
& Q_{1}^{*}= \pm \epsilon a_{5} \gamma-\epsilon^{3} a_{5}\left(a_{5}^{2}+3 a_{5} a_{10}-2 b_{1}-2 b_{10}\right) \gamma^{2}+O\left(\epsilon^{4}\right), \\
& Q_{2}^{*}=\mp \gamma+\epsilon^{2}\left(a_{5}^{2}-2 b_{1}\right) \gamma^{2}+O\left(\epsilon^{4}\right), \\
& P_{1}^{*}=0, \\
& P_{2}^{*}=0,
\end{aligned}
$$


and Hamiltonian (6) is given by

$$
\mathcal{H}_{\epsilon}(Q, P)=\mathcal{H}_{0}+\epsilon \mathcal{H}_{1}+\epsilon^{2} \mathcal{H}_{2}
$$

where

$$
\begin{aligned}
& \mathcal{H}_{0}=\frac{1}{2 Q_{2}}\left[\gamma^{2}+Q_{2}\left(Q_{2}+Q_{1}^{2}+P_{1}^{2}+4 Q_{2} P_{2}^{2}\right)\right], \\
& \mathcal{H}_{1}=Q_{1}\left(a_{5} Q_{2}+a_{10} Q_{1}^{2}\right), \\
& \mathcal{H}_{2}=b_{1} Q_{2}^{2}+b_{10} Q_{1}^{2} Q_{2}+b_{15} Q_{1}^{4} .
\end{aligned}
$$

Now, we shift the origin to the equilibrium points $\mathcal{O}^{j}$ and rescale time, dividing the Hamiltonian by $\epsilon^{1 / 2}$ and performing the symplectic linear change

$Q_{1}=\epsilon^{1 / 4} \bar{Q}_{1}+Q_{1}^{*}, \quad Q_{2}=\epsilon^{1 / 4} \bar{Q}_{2}+Q_{2}^{*}, \quad P_{1}=\epsilon^{1 / 4} \bar{P}_{1}+P_{1}^{*}, \quad P_{2}=\epsilon^{1 / 4} \bar{P}_{2}+P_{2}^{*}$,

where $Q_{1}^{*}, Q_{2}^{*}, P_{1}^{*}, P_{2}^{*}$ stand for the coordinates of the equilibria given in (17) after truncation of terms of order $O\left(\epsilon^{4}\right)$. Next, we expand the resulting Hamiltonian around the origin in powers of $\epsilon$, obtaining

$$
\mathcal{H}_{\epsilon}(\bar{Q}, \bar{P})=\mathcal{H}_{\epsilon, 0}+\mathcal{H}_{\epsilon, 2}+\mathcal{H}_{\epsilon, 3}+\mathcal{H}_{\epsilon, 4}+O\left(\epsilon^{3 / 4}\right),
$$

where

$$
\begin{aligned}
\mathcal{H}_{\epsilon, 0}= & \mp \epsilon^{-1 / 2} \frac{\gamma}{2}\left[2 \pm \epsilon^{2}\left(a_{5}^{2}-2 b_{1}\right) \gamma\right]+O\left(\epsilon^{7 / 2}\right), \\
\mathcal{H}_{\epsilon, 2}= & \frac{1}{2 \gamma}\left[\gamma\left(\bar{Q}_{1}^{2}+\bar{P}_{1}^{2}\right) \mp\left(\bar{Q}_{2}^{2}+4 \gamma^{2} \bar{P}_{2}^{2}\right)\right]+\epsilon a_{5} \bar{Q}_{1} \bar{Q}_{2} \\
& +\epsilon^{2} \frac{\gamma}{2}\left[ \pm 2\left(3 a_{5} a_{10}-b_{10}\right) \bar{Q}_{1}^{2}+\left(-3 a_{5}^{2}+8 b_{1}\right) \bar{Q}_{2}^{2}+4\left(a_{5}^{2}-b_{1}\right) \gamma \bar{P}_{2}^{2}\right] \\
& \pm 2 \epsilon^{3} a_{5} b_{10} \gamma \bar{Q}_{1} \bar{Q}_{2}+O\left(\epsilon^{4}\right),
\end{aligned}
$$

and $\mathcal{H}_{\epsilon, 3}, \mathcal{H}_{\epsilon, 4}$ are homogeneous polynomials of degree 3 and 4 in $(\bar{Q}, \bar{P})$, respectively. These two Hamiltonians depend upon $\epsilon$. The remainder $O\left(\epsilon^{3 / 4}\right)$ includes terms of degree at least five in $(\bar{Q}, \bar{P})$ while the lowest power in $\epsilon$ is $3 / 4$. When $\epsilon=0$, the quadratic part of $\mathcal{H}_{\epsilon}$ is in 2:1 resonance, as it also happens when only cubic axially-symmetric perturbations are considered [31]. 
The eigenvalues associated to $\mathcal{H}_{\epsilon, 2}$ are $\pm i \lambda_{1}, \pm i \lambda_{2}$, where

$$
\lambda_{1}=2 \pm \epsilon^{2} c_{1} \gamma+O\left(\epsilon^{4}\right), \quad \lambda_{2}=1 \pm \epsilon^{2} c_{2} \gamma+O\left(\epsilon^{4}\right),
$$

with

$$
c_{1}=\frac{1}{3}\left(5 a_{5}^{2}-18 b_{1}\right), \quad c_{2}=\frac{1}{3}\left(2 a_{5}^{2}+9 a_{5} a_{10}-3 b_{10}\right) .
$$

Thus, for a sufficiently small $\epsilon$ the equilibria $\mathcal{O}^{j}$ are not in 1:-1 resonance and therefore they are parametrically stable on the space $\mathcal{M}_{\gamma}$. Then, the periodic solutions reconstructed from $\mathcal{O}^{j}$ are linearly stable, see more details in [71].

The periods of the solutions are computed by calculating $2 \pi\left|\partial \mathcal{H}_{\epsilon}(Q, P) / \partial \gamma\right|^{-1}$, evaluating the result at $\left(Q_{1}^{*}, Q_{2}^{*}, P_{1}^{*}, P_{2}^{*}\right)$ and expanding it in terms of $\epsilon$ around 0 . We end up with $2 \pi\left[1 \mp \epsilon^{2}\left|a_{5}^{2}-2 b_{1}\right| \gamma\right]+O\left(\epsilon^{4}\right)$.

The characteristic multipliers corresponding to these periodic solutions will be computed in Section 4.

Now, let us consider periodic solutions whose projection in configuration space Oxyz are motions on the $O z$-axis. When $\gamma=0$ the subspace of $\mathbb{R}^{6}$ given by $i_{1}=i_{2}=i_{3}=i_{4}=0$ is invariant under the axial symmetry because the plane $\Pi=\operatorname{span}\left\{e_{3}, e_{6}\right\}$ with $e_{2}=(0,0,1,0,0,0)$ and $e_{6}=(0,0,0,0,0,1)$ is invariant. Under these conditions system (13) is reduced to

$$
\begin{aligned}
& i_{5}^{\prime}=i_{6}, \\
& i_{6}^{\prime}=-i_{5}\left(1+3 \epsilon a_{10} i_{5}+4 \epsilon^{2} b_{15} i_{5}^{2}\right) .
\end{aligned}
$$

Note that it is an anharmonic oscillator on the $O z Z$-plane. The solutions of this system can be obtained explicitly in terms of elliptic integrals. Some of these motions correspond to periodic solutions of the Hamiltonian system derived from (6). Nevertheless this type of periodic motions will be analysed after normalising when studying the dynamics of the reduced system in $\mathbb{C P}_{h}^{2}$ and also in the twice reduced 
space as we shall get easier expressions; so, we postpone their study to Sections 4 and 5 .

3.3. KAM tori of dimension 3 from $\mathcal{M}_{\gamma}$. In order to prove the existence of KAM tori, we define the auxiliary constants

$$
\begin{aligned}
c_{3} & =\frac{4}{3} a_{5}\left(a_{5}-3 a_{10}\right), & m & =3\left(c_{1}-2 c_{2}\right), \\
\Delta_{1} & =\frac{1}{m}\left[3\left(c_{2}+c_{3}\right)^{2}+2 c_{2} m\right], & \Delta_{2} & =\frac{3}{4 m}\left[\left(c_{2}+c_{3}\right)^{2}-m\left(5 a_{10}^{2}-2 b_{15}\right)\right],
\end{aligned}
$$

with $c_{1}$ and $c_{2}$ given in (23).

Theorem 3.2. When $c_{1} m \neq 0$ and $\epsilon>0$ is sufficiently small, there are invariant KAM 3-tori for Hamiltonian system (6) around the prograde and retrograde nearly circular periodic solutions on the Oxy-plane. These invariant tori form a majority in the sense that the excluding measure for the existence of the quasi-periodic invariant tori is of order $\epsilon$.

Proof Considering Hamiltonian (20), we put its quadratic part, $\mathcal{H}_{\epsilon, 2}$, in diagonal (normal) form. For that, we construct a linear complex symplectic change of coordinates $\left(\bar{Q}_{1}, \bar{Q}_{2}, \bar{P}_{1}, \bar{P}_{2}\right) \longrightarrow\left(q_{1}, q_{2}, p_{1}, p_{2}\right)$ by means of the eigenvectors associated to $\mathcal{H}_{\epsilon, 2}$, see details in [45, 50], and apply it to Hamiltonian (20). The resulting Hamiltonian is

$$
\mathcal{H}_{\epsilon}(q, p)=\mathcal{H}_{\epsilon, 0}+i\left(\lambda_{1} q_{1} p_{1}+\lambda_{2} q_{2} p_{2}\right)+\mathcal{H}_{\epsilon, 3}+\mathcal{H}_{\epsilon, 4}+O\left(\epsilon^{3 / 4}\right)
$$

where $\mathcal{H}_{\epsilon, 3}, \mathcal{H}_{\epsilon, 4}$ are homogeneous polynomials of degree 3 and 4 in the new coordinates $q$ and $p$, respectively. Both Hamiltonians depend on $\epsilon$ but in such a way that the bigger terms in $\mathcal{H}_{\epsilon, 3}$ with respect to $\epsilon$ are of order $\epsilon^{1 / 4}$ while the bigger terms in $\mathcal{H}_{\epsilon, 4}$ with respect to $\epsilon$ are of order $\epsilon^{1 / 2}$. This gives an idea of the correctness of the ordering of the Hamiltonians $\mathcal{H}_{\epsilon, k}$ in (26) for the forthcoming step.

As the next step, we perform a Lie-Deprit transformation, see for example [18], with the aim of simplifying the Hamiltonian function. This normal form transformation is made up to terms of degree four in the coordinates $q$ and $p$, that is, 
two steps in the Lie triangle process are performed. After this, we arrive at the following Hamiltonian:

$$
\mathcal{H}_{\epsilon}(q, p)=\mathcal{H}_{\epsilon, 0}+i\left(\lambda_{1} q_{1} p_{1}+\lambda_{2} q_{2} p_{2}\right)+\epsilon^{5 / 2} \mathcal{H}_{4}+O\left(\epsilon^{3}\right)
$$

where $\mathcal{H}_{\epsilon, 0}$ is given in (21), $\lambda_{1}, \lambda_{2}$ have been introduced in (22) and $\mathcal{H}_{4}$ is a homogeneous polynomial of degree 4 in $(q, p)$ in normal form with respect to the quadratic part and independent of $\epsilon$. For the existence of the Lie-Deprit transformation it is necessary to assume that $m \neq 0$ because this term is present at the denominators of the generating function built in the process.

With the aim of applying a KAM-type result, we express the normalised Hamiltonian $\mathcal{H}_{\epsilon}$ in $(27)$ in adequate action-angle coordinates $(I, \phi)=\left(I_{1}, I_{2}, \phi_{1}, \phi_{2}\right)$. They are defined by means of the symplectic transformation

$$
\begin{array}{ll}
q_{1}=\sqrt{I_{1}}\left(\cos \phi_{1}-i \sin \phi_{1}\right), & q_{2}=\sqrt{I_{2}}\left(\cos \phi_{2}-i \sin \phi_{2}\right), \\
p_{1}=\sqrt{I_{1}}\left(\sin \phi_{1}-i \cos \phi_{1}\right), & p_{2}=\sqrt{I_{2}}\left(\sin \phi_{2}-i \cos \phi_{2}\right) .
\end{array}
$$

We also identify $\gamma$ with a third action recalling that $\gamma=N$. As we prefer to apply a KAM-type result to the full Hamiltonian, $\mathcal{H}_{\epsilon}$, we undo the operation we have performed to pass from the unnormalised $\mathcal{H}_{\epsilon}$ to the normal form (with the same name), i.e. we rescale time by multiplying $\mathcal{H}_{\epsilon}$ by $\epsilon^{1 / 2}$. Then, after some simplifications the resulting Hamiltonian in action-angle coordinates reads as

$$
\begin{aligned}
\mathcal{H}_{\epsilon}(\phi, N, I)= & h_{0}(N)+\eta h_{1}\left(N, I_{1}, I_{2}\right)+\eta^{4} h_{2}\left(N, I_{1}, I_{2}\right)+\eta^{5} h_{3}\left(N, I_{1}, I_{2}\right) \\
& +\eta^{6} h_{4}\left(N, I_{1}, I_{2}\right)+O\left(\eta^{7}\right)
\end{aligned}
$$

where $\eta=\epsilon^{1 / 2}$ and

$$
\begin{aligned}
& h_{0}=\mp N, \quad h_{1}=2 I_{1}+I_{2}, \quad h_{2}=-\frac{1}{2}\left(a_{5}^{2}-2 b_{1}\right) N^{2}, \\
& h_{3}= \pm\left(c_{1} I_{1}+c_{2} I_{2}\right) N, \quad h_{4}=-c_{1} I_{1}^{2}-\Delta_{1} I_{1} I_{2}+\Delta_{2} I_{2}^{2},
\end{aligned}
$$

with $m \neq 0$. We emphasise that (29) stands for Hamiltonian function (6) in neighbourhoods of the periodic solutions established in Theorem 3.1. Note also that the 
terms $h_{j}$ are independent of the small parameter $\eta$, indeed they only depend on the $a_{i}$ and $b_{j}$.

As the Hamiltonian is highly properly degenerate we cannot apply a classical result on KAM theory, see [3]. Thus we apply a recent result of Han, Li and Yi [39]. Following the notation in Han-Li-Yi's paper, $a=4, n_{0}=1, n_{1}=n_{2}=$ $n_{3}=n_{4}=n=3, m_{1}=1, m_{2}=4, m_{3}=5, m_{4}=6, y^{n_{0}}=N, y^{n_{1}}=y^{n_{2}}=y^{n_{3}}=$ $y^{n_{4}}=\left(N, I_{1}, I_{2}\right)$. The vector of frequencies has dimension 9 and is given by

$$
\Omega\left(N, I_{1}, I_{2}\right)=\left(\frac{\partial h_{0}}{\partial N}, \frac{\partial h_{1}}{\partial I_{1}}, \frac{\partial h_{1}}{\partial I_{2}}, \frac{\partial h_{2}}{\partial I_{1}}, \frac{\partial h_{2}}{\partial I_{2}}, \frac{\partial h_{3}}{\partial I_{1}}, \frac{\partial h_{3}}{\partial I_{2}}, \frac{\partial h_{4}}{\partial I_{1}}, \frac{\partial h_{4}}{\partial I_{2}}\right)
$$

and the $9 \times 4$ matrix whose columns are $\Omega, \partial \Omega / \partial N, \partial \Omega / \partial I_{1}$ and $\partial \Omega / \partial I_{2}$ reads as

$$
\left[\begin{array}{cccc}
\Omega_{1} & \frac{\partial \Omega_{1}}{\partial N} & \frac{\partial \Omega_{1}}{\partial I_{1}} & \frac{\partial \Omega_{1}}{\partial I_{2}} \\
\Omega_{2} & \frac{\partial \Omega_{2}}{\partial N} & \frac{\partial \Omega_{2}}{\partial I_{1}} & \frac{\partial \Omega_{2}}{\partial I_{2}} \\
\Omega_{3} & \frac{\partial \Omega_{3}}{\partial N} & \frac{\partial \Omega_{3}}{\partial I_{1}} & \frac{\partial \Omega_{3}}{\partial I_{2}} \\
\Omega_{4} & \frac{\partial \Omega_{4}}{\partial N} & \frac{\partial \Omega_{4}}{\partial I_{1}} & \frac{\partial \Omega_{4}}{\partial I_{2}} \\
\Omega_{5} & \frac{\partial \Omega_{5}}{\partial N} & \frac{\partial \Omega_{5}}{\partial I_{1}} & \frac{\partial \Omega_{5}}{\partial I_{2}} \\
\Omega_{6} & \frac{\partial \Omega_{6}}{\partial N} & \frac{\partial \Omega_{6}}{\partial I_{1}} & \frac{\partial \Omega_{6}}{\partial I_{2}} \\
\Omega_{7} & \frac{\partial \Omega_{7}}{\partial N} & \frac{\partial \Omega_{7}}{\partial I_{1}} & \frac{\partial \Omega_{7}}{\partial I_{2}} \\
\Omega_{8} & \frac{\partial \Omega_{8}}{\partial N} & \frac{\partial \Omega_{8}}{\partial I_{1}} & \frac{\partial \Omega_{8}}{\partial I_{2}} \\
\Omega_{9} & \frac{\partial \Omega_{9}}{\partial N} & \frac{\partial \Omega_{9}}{\partial I_{1}} & \frac{\partial \Omega_{9}}{\partial I_{2}}
\end{array}\right]=\left[\begin{array}{cccc}
0 & 0 & 0 \\
0 & 0 & 0 & 0 \\
0 & 0 & 0 & 0 \\
\pm c_{1} N & \pm c_{1} & 0 & 0 \\
\pm c_{2} N & \pm c_{2} & 0 & 0 \\
-2 c_{1} I_{1}-\Delta_{1} I_{2} & 0 & -2 c_{1} & -\Delta_{1} \\
-\Delta_{1} I_{1}+2 \Delta_{2} I_{2} & 0 & -\Delta_{1} & 2 \Delta_{2}
\end{array}\right]
$$

When $c_{1} \neq 0$ the rank of the matrix is 4 while the rank is two when $c_{1}=0$. Therefore, Han-Li-Yi's Theorem guarantees the existence of KAM tori of dimension 3 around the circular-equatorial periodic solutions provided $c_{1} \neq 0$. Setting $b=$ $\sum_{j=1}^{a} m_{j}\left(n_{j}-n_{j-1}\right)$, we obtain $b=2$. The maximum order of the partial derivatives involved in the computation of the $9 \times 4$ matrix is $s=1$. As the perturbation is in an order $O\left(\eta^{8}\right)$ and $\eta^{s b+\mu}=\eta^{2+\mu}>\eta^{7}$ (for a pre-fixed small positive constant 
$\mu<1 / 5)$, the excluding measure for the existence of the quasi-periodic invariant tori is of order $\eta^{b}=\eta^{2}=\epsilon$.

Remark 1. When $m=0$ we have considered a Taylor series of the equilibria $\mathcal{O}^{j}$ as in (15) but carrying out the computations up to order $O\left(\epsilon^{4}\right)$. Proceeding in the same way as in the proof of Theorem 3.2, we conclude the existence of KAM tori whenever $c_{1} \tilde{m} \neq 0$ with $\tilde{m}=49 a_{5}^{3}-81 a_{5}^{2} a_{10}-243 a_{5} a_{10}^{2}-162 a_{5} b_{1}-486 a_{10} b_{1}-162 a_{5} b_{15} \neq 0$. We have not kept on analysing what happens in the case $\tilde{m}=0$.

\section{REduCtion by the OSCILlator Symmetry AND RECONSTRUCTION}

\subsection{Normalisation and reduction by the oscillator symmetry. The iso-} tropic harmonic oscillator (3) is invariant under the so called oscillator symmetry, although Hamiltonian (6) is not invariant under this symmetry, in general. By normalisation and truncation of higher order terms we extend the oscillator symmetry to the whole (truncated) Hamiltonian. In the following paragraphs we describe the reduction induced by the oscillator symmetry. Details can be seen in the recent book [10].

A level set $h$ of Hamiltonian (3) is the 5-dimensional sphere $S_{h}^{5} \subset \mathbb{C}^{3}$ of radius $\sqrt{2 h}$, where we can define complex coordinates $w_{1}=x+i X, w_{2}=y+i Y, w_{3}=$ $z+i Z$. The one-dimensional unitary group $U(1)=\left\{e^{i t} \mid t \in[0,2 \pi)\right\}$, that is isomorphic to the sphere $S^{1}$, acts on $\mathbb{C}^{3}$ by complex multiplication, i.e. it defines a map

$$
\begin{aligned}
\varphi: U(1) \times \mathbb{C}^{3} & \rightarrow \mathbb{C}^{3} \\
\left(e^{i t}, w\right) & \mapsto e^{i t} w
\end{aligned}
$$

where $w=\left(w_{1}, w_{2}, w_{3}\right)$. Its infinitesimal generator defines precisely the fundamental vector field associated to Hamiltonian (3) and its orbits are the trajectories of the harmonic oscillator. The space of orbits of fixed energy $h$ is the quotient space $S_{h}^{5} / U(1)$. Such a space is the 4 -dimensional complex projective space $\mathbb{C P}_{h}^{2}[60]$, that is characterised by the following nine linearly independent invariants, see for 
example [70, 34]:

$$
\begin{array}{lll}
\rho_{1}=x^{2}+X^{2}, & \rho_{2}=z^{2}+Z^{2}, & \rho_{3}=y^{2}+Y^{2}, \\
\rho_{4}=x z+X Z, & \rho_{5}=x y+X Y, & \rho_{6}=y z+Y Z, \\
\rho_{7}=x Z-z X, & \rho_{8}=x Y-y X, & \rho_{9}=z Y-y Z .
\end{array}
$$

The first six invariants describe the orbit in the plane of motion. The other invariants, $\rho_{7}, \rho_{8}$ and $\rho_{9}$, give us the position of the plane in the space because they are the components of the angular momentum vector, except for the sign in $\rho_{7}$ and $\rho_{9}$. The linear and quadratic constraints among the invariants are the following:

$$
\begin{array}{cll}
\rho_{1}+\rho_{2}+\rho_{3}=2 h, \quad \rho_{1} \geq 0, \rho_{2} \geq 0, & \rho_{3} \geq 0, \\
\rho_{1} \rho_{2}=\rho_{4}^{2}+\rho_{7}^{2}, & \rho_{1} \rho_{3}=\rho_{5}^{2}+\rho_{8}^{2}, \quad \rho_{2} \rho_{3}=\rho_{6}^{2}+\rho_{9}^{2}, \\
\rho_{1} \rho_{6}=\rho_{4} \rho_{5}+\rho_{7} \rho_{8}, & \rho_{2} \rho_{8}=\rho_{4} \rho_{9}+\rho_{6} \rho_{7}, & \rho_{3} \rho_{4}=\rho_{5} \rho_{6}+\rho_{8} \rho_{9}, \\
\rho_{4} \rho_{6}=\rho_{2} \rho_{5}+\rho_{7} \rho_{9}, & \rho_{4} \rho_{8}=\rho_{1} \rho_{9}+\rho_{5} \rho_{7}, & \rho_{6} \rho_{8}=\rho_{3} \rho_{7}+\rho_{5} \rho_{9} .
\end{array}
$$

Since $\mathbb{C P}_{h}^{2}$ is a 4 -dimensional compact symplectic space it is characterised by the nine invariants subject to five fundamental relations and the inequalities $\rho_{i} \geq 0$, $i=1,2,3$. There is some freedom in choosing the fundamental relations, but one has to select $\rho_{1}+\rho_{2}+\rho_{3}=2 h$ and four more from the second, third or fourth rows of (31).

Table 2 shows the action of the Poisson bracket on the invariants. It has been obtained in $[70,31,34]$.

\begin{tabular}{|c||c|c|c|c|c|c|c|c|c|}
\hline$\left\{\rho_{j}, \rho_{k}\right\}$ & $\rho_{1}$ & $\rho_{2}$ & $\rho_{3}$ & $\rho_{4}$ & $\rho_{5}$ & $\rho_{6}$ & $\rho_{7}$ & $\rho_{8}$ & $\rho_{9}$ \\
\hline \hline$\rho_{1}$ & 0 & 0 & 0 & $2 \rho_{7}$ & $2 \rho_{8}$ & 0 & $-2 \rho_{4}$ & $-2 \rho_{5}$ & 0 \\
\hline$\rho_{2}$ & 0 & 0 & 0 & $-2 \rho_{7}$ & 0 & $2 \rho_{9}$ & $2 \rho_{4}$ & 0 & $-2 \rho_{6}$ \\
\hline$\rho_{3}$ & 0 & 0 & 0 & 0 & $-2 \rho_{8}$ & $-2 \rho_{9}$ & 0 & $2 \rho_{5}$ & $2 \rho_{6}$ \\
\hline$\rho_{4}$ & $-2 \rho_{7}$ & $2 \rho_{7}$ & 0 & 0 & $\rho_{9}$ & $\rho_{8}$ & $\rho_{1}-\rho_{2}$ & $-\rho_{6}$ & $-\rho_{5}$ \\
\hline$\rho_{5}$ & $-2 \rho_{8}$ & 0 & $2 \rho_{8}$ & $-\rho_{9}$ & 0 & $\rho_{7}$ & $-\rho_{6}$ & $\rho_{1}-\rho_{3}$ & $\rho_{4}$ \\
\hline$\rho_{6}$ & 0 & $-2 \rho_{9}$ & $2 \rho_{9}$ & $-\rho_{8}$ & $-\rho_{7}$ & 0 & $\rho_{5}$ & $\rho_{4}$ & $\rho_{2}-\rho_{3}$ \\
\hline$\rho_{7}$ & $2 \rho_{4}$ & $-2 \rho_{4}$ & 0 & $\rho_{2}-\rho_{1}$ & $\rho_{6}$ & $-\rho_{5}$ & 0 & $\rho_{9}$ & $-\rho_{8}$ \\
\hline$\rho_{8}$ & $2 \rho_{5}$ & 0 & $-2 \rho_{5}$ & $\rho_{6}$ & $\rho_{3}-\rho_{1}$ & $-\rho_{4}$ & $-\rho_{9}$ & 0 & $\rho_{7}$ \\
\hline$\rho_{9}$ & 0 & $2 \rho_{6}$ & $-2 \rho_{6}$ & $\rho_{5}$ & $-\rho_{4}$ & $\rho_{3}-\rho_{2}$ & $\rho_{8}$ & $-\rho_{7}$ & 0 \\
\hline
\end{tabular}

TABLE 2. Poisson brackets among the invariants $\rho_{i}$. 
The reduced space $\mathbb{C P}_{h}^{2}$ is a symplectic manifold and the reduction process is regular, see $[53,51]$.

In order to perform this reduction explicitly, we first normalise Hamiltonian (6) with respect to $\mathcal{H}_{0}$ in terms of symplectic complex coordinates including homogeneous polynomials of degree 4 in these coordinates, similarly to what we did in $[31,34]$. We use the method of Deprit [18], performing two steps in the Lie triangle process.

Next, we express the resulting Hamiltonian as a function of the invariants by applying the division algorithm for multivariate polynomials $[65,14]$. In order to achieve it we calculate the Gröbner basis of the invariants together with their relations, then we divide the normalised Hamiltonian with respect to this Gröbner basis and take the remainder in the division. This remainder is an expression given as a function of the invariants that is invariant with respect to $\mathcal{H}_{0}$. This method can be applied to check whether a certain expression (usually a polynomial written in some collection of variables) does belong or does not belong to the ideal generated by another set of polynomials.

After some simplifications, the normalised reduced Hamiltonian takes the form

$$
\begin{aligned}
\mathcal{H}_{\epsilon}=\frac{1}{2}\left(\rho_{1}+\rho_{2}+\rho_{3}\right)+\frac{\epsilon^{2}}{2}[ & d_{1}\left(\rho_{1}+\rho_{2}+\rho_{3}\right)^{2}+d_{2}\left(\rho_{1}+\rho_{2}+\rho_{3}\right) \rho_{2} \\
& \left.+d_{3}\left(\rho_{4}^{2}+\rho_{6}^{2}\right)+d_{4}\left(\rho_{7}^{2}+\rho_{9}^{2}\right)+d_{5} \rho_{8}^{2}\right]+O\left(\epsilon^{4}\right),
\end{aligned}
$$

where

$$
\begin{aligned}
& d_{1}=\frac{1}{24}\left(-5 a_{5}^{2}+18 b_{1}\right) \\
& d_{2}=\frac{1}{24}\left(5 a_{5}^{2}-45 a_{10}^{2}-18 b_{1}+18 b_{15}\right) \\
& d_{3}=\frac{1}{8}\left(-5 a_{5}^{2}-10 a_{5} a_{10}+15 a_{10}^{2}-6 b_{1}+6 b_{10}-6 b_{15}\right) \\
& d_{4}=\frac{1}{8}\left(3 a_{5}^{2}-14 a_{5} a_{10}+15 a_{10}^{2}-6 b_{1}+2 b_{10}-6 b_{15}\right) \\
& d_{5}=-\frac{1}{6}\left(a_{5}^{2}+6 b_{1}\right)
\end{aligned}
$$


Now we fix $\mathcal{H}_{0}=\left(\rho_{1}+\rho_{2}+\rho_{3}\right) / 2=h>0$, rescale $\mathcal{H}_{\epsilon}$ changing the time and drop constant terms, obtaining

$$
\overline{\mathcal{H}}_{\epsilon}=2 d_{2} h \rho_{2}+d_{3}\left(\rho_{4}^{2}+\rho_{6}^{2}\right)+d_{4}\left(\rho_{7}^{2}+\rho_{9}^{2}\right)+d_{5} \rho_{8}^{2}+O\left(\epsilon^{2}\right) .
$$

Applying Liouville's Theorem to $\overline{\mathcal{H}}_{\epsilon}$ and taking into account the Poisson brackets in Table 2, the equations of motion associated to Hamiltonian (34), after truncation of order higher than one in $\epsilon$, are

$$
\begin{aligned}
& \dot{\rho}_{1}=4\left[\left(d_{3}-d_{4}\right) \rho_{4} \rho_{7}-d_{5} \rho_{5} \rho_{8}\right], \\
& \dot{\rho}_{2}=-4\left(d_{3}-d_{4}\right)\left(\rho_{4} \rho_{7}-\rho_{6} \rho_{9}\right), \\
& \dot{\rho}_{3}=4\left[d_{5} \rho_{5} \rho_{8}-\left(d_{3}-d_{4}\right) \rho_{6} \rho_{9}\right], \\
& \dot{\rho}_{4}=4\left(d_{2}+d_{4}\right) h \rho_{7}-2 d_{4}\left(2 \rho_{2} \rho_{7}+\rho_{3} \rho_{7}+\rho_{5} \rho_{9}\right)+2\left(d_{3}-d_{5}\right) \rho_{6} \rho_{8}, \\
& \dot{\rho}_{5}=4 d_{5} h \rho_{8}+2\left(d_{3}-d_{4}\right)\left(\rho_{6} \rho_{7}-\rho_{4} \rho_{9}\right)-2 d_{5}\left(\rho_{2} \rho_{8}+2 \rho_{3} \rho_{8}\right), \\
& \dot{\rho}_{6}=-4 d_{2} h \rho_{9}+2 d_{4}\left(\rho_{5} \rho_{7}+\rho_{2} \rho_{9}-\rho_{3} \rho_{9}\right)-2\left(d_{3}-d_{5}\right) \rho_{4} \rho_{8}, \\
& \dot{\rho}_{7}=-4\left(d_{2}+d_{3}\right) h \rho_{4}+2 d_{3}\left(2 \rho_{2} \rho_{4}+\rho_{3} \rho_{4}-\rho_{5} \rho_{6}\right)-2\left(d_{4}-d_{5}\right) \rho_{8} \rho_{9}, \\
& \dot{\rho}_{8}=0, \\
& \dot{\rho}_{9}=4 d_{2} h \rho_{6}+2 d_{3}\left(\rho_{4} \rho_{5}-\rho_{2} \rho_{6}+\rho_{3} \rho_{6}\right)+2\left(d_{4}-d_{5}\right) \rho_{7} \rho_{8},
\end{aligned}
$$

where the dots refer to derivation with respect to the rescaled time.

4.2. Periodic solutions reconstructed from $\mathbb{C P}_{h}^{2}$. Under some conditions of non-degeneracy the equilibria of system (35) correspond with families of periodic solutions of Hamiltonian (6). System (35) can be simplified, for instance, by eliminating some of the variables $\rho_{j}$. Even then, it is not easy to discuss all the equilibria from a direct analysis of (35). So, we have to resort to other approaches.

Topology arguments provide a lower bound in the number of periodic solutions of a given Hamiltonian system. On the one hand, the Lusternik-Schnirelmann category of $\mathbb{C P}_{h}^{2}$ is 2 (see [13]) and this space is compact and simply connected, thus 2 is a lower bound of the number of periodic solutions. On the other hand, the 
Euler-Poincaré characteristic of $\mathbb{C P}_{h}^{2}$ is 3 and when the normalised Hamiltonian (32) after truncating terms of degree higher than 4 in Cartesian coordinates is a Morse function then, the full Hamiltonian (6) has at least 3 families of periodic solutions (see [71] and references therein). Weinstein's Theorem [67, 68], that provides a lower bound of the number of periodic solutions of a certain flow in terms of the LusternikSchnirelmann category of the reduced space, also states that Hamiltonian (6) has at least 3 periodic solutions.

Proceeding similarly as we did with the axial symmetry reduction in Section 3 we focus on two special solutions: (i) periodic solutions in $T^{*} \mathbb{R}^{3}$ whose projections in configuration space are circular solutions on the $O x y$-plane, and that we call circular-equatorial solutions; and (ii) periodic solutions whose projection in configuration space $O x y z$ are rectilinear trajectories on the $O z$-axis. Recall that case (ii) was difficult to be handled in $\mathcal{M}_{\gamma}$ and its discussion was postposed to this section. The rest of the solutions will be considered in the twice reduced space in Section 5 .

We establish the first result regarding the periodic solutions of type (i) according to the discussion of the previous paragraph.

Theorem 4.1. When $\left(2 d_{2}+d_{3}+d_{4}-d_{5}\right) d_{5} \neq 0$ the points in $\mathbb{C P}_{h}^{2}$ with coordinates $\mathcal{O}_{C}^{1}=(h, 0, h, 0,0,0,0, h, 0)$ and $\mathcal{O}_{C}^{2}=(h, 0, h, 0,0,0,0,-h, 0)$ are linearly stable equilibria (centres) of system (35) and, for a sufficiently small $\epsilon>0$, they give rise to families of periodic solutions of Hamiltonian (6) parametrised by $h>0$ whose projections in configuration space are close to (retrograde and prograde, respectively) circular trajectories on the Oxy-plane. Moreover, when $2 d_{2}+d_{3}+d_{4}-3 d_{5} \neq$ 0 the periodic solutions are linearly stable with characteristic multipliers $1,1,1 \pm$ $4 \pi i \epsilon^{2}\left(2 d_{2}+d_{3}+d_{4}-d_{5}\right) h+O\left(\epsilon^{4}\right), 1 \pm 8 \pi i \epsilon^{2} d_{5} h+O\left(\epsilon^{4}\right)$ and periods $T(\epsilon)=2 \pi[1 \mp$ $\left.\epsilon^{2}\left|4 d_{1}+d_{5}\right| h\right]+O\left(\epsilon^{4}\right)$.

Proof It can be easily checked that $\mathcal{O}_{C}^{1}$ and $\mathcal{O}_{C}^{2}$ are isolated zeroes of system (35). Taking into account formulae (30), we observe that they correspond to families of solutions on the $O x y$-plane because $\rho_{2}=\rho_{4}=\rho_{6}=\rho_{7}=\rho_{9}=0$. Moreover, they are of circular type because $\rho_{7}^{2}+\rho_{8}^{2}+\rho_{9}^{2}=h^{2}$, see [70,34] for more information. 
In order to analyse the parametric stability of $\mathcal{O}_{C}^{1}$ and $\mathcal{O}_{C}^{2}$ we have to introduce convenient local symplectic coordinates in $\mathbb{C P}_{h}^{2}$. We describe the method we follow to find this set of coordinates. First, we express the normal form (34) in rectangular variables through formulae (30) and then in Poincaré action-angle variables $(I, \phi)=$ $\left(I_{1}, I_{2}, I_{3}, \phi_{1}, \phi_{2}, \phi_{3}\right)$, that are defined by

$$
\begin{gathered}
x=\sqrt{2 I_{1}} \cos \phi_{1}, \quad y=\sqrt{2 I_{2}} \cos \phi_{2}, \quad z=\sqrt{2 I_{3}} \cos \phi_{3}, \\
X=\sqrt{2 I_{1}} \sin \phi_{1}, \quad Y=\sqrt{2 I_{2}} \sin \phi_{2}, \quad Z=\sqrt{2 I_{3}} \sin \phi_{3} .
\end{gathered}
$$

Note that the zeroth-order term (3) of Hamiltonian (6) reads as $I_{1}+I_{2}+I_{3}$. At this point we introduce a linear canonical change of variables from $\left(I_{1}, I_{2}, I_{3}, \phi_{1}, \phi_{2}, \phi_{3}\right)$ to $\left(J_{1}, J_{2}, J_{3}, \theta_{1}, \theta_{2}, \theta_{3}\right)$ in such a way that the zeroth-order term be one of the new actions, for instance $J_{1}=I_{1}+I_{2}+I_{3}$. Then, the normal form will depend on two combinations of the angles, namely, $\theta_{2}=\phi_{2}-\phi_{1}$ and $\theta_{3}=\phi_{3}-\phi_{1}$. For completeness we set $J_{2}=I_{2}, J_{3}=I_{3}$ and $\theta_{1}=\phi_{1}$. Finally, we introduce rectangular canonical coordinates from $\left(J_{2}, J_{3}, \theta_{2}, \theta_{3}\right)$ as:

$Q_{1}=\sqrt{2 J_{2}} \cos \theta_{2}, \quad Q_{2}=\sqrt{2 J_{3}} \cos \theta_{3}, \quad P_{1}=\sqrt{2 J_{2}} \sin \theta_{2}, \quad P_{2}=\sqrt{2 J_{3}} \sin \theta_{3}$.

The expression of these rectangular variables as functions of the invariants is

$$
Q_{1}=\frac{\rho_{4}}{\sqrt{\rho_{1}}}, \quad Q_{2}=\frac{\rho_{5}}{\sqrt{\rho_{1}}}, \quad P_{1}=\frac{\rho_{7}}{\sqrt{\rho_{1}}}, \quad P_{2}=\frac{\rho_{8}}{\sqrt{\rho_{1}}}
$$

that is not defined for $\rho_{1}=0$. The inverse of (37) is

$$
\begin{array}{lc}
\rho_{1}=2 h-\left(Q_{1}^{2}+Q_{2}^{2}+P_{1}^{2}+P_{2}^{2}\right), & \rho_{2}=Q_{1}^{2}+P_{1}^{2}, \quad \rho_{3}=Q_{2}^{2}+P_{2}^{2}, \\
\rho_{4}=Q_{1} \sqrt{2 h-\left(Q_{1}^{2}+Q_{2}^{2}+P_{1}^{2}+P_{2}^{2}\right)}, & \rho_{5}=Q_{2} \sqrt{2 h-\left(Q_{1}^{2}+Q_{2}^{2}+P_{1}^{2}+P_{2}^{2}\right)}, \\
\rho_{6}=Q_{1} Q_{2}+P_{1} P_{2}, & \rho_{7}=P_{1} \sqrt{2 h-\left(Q_{1}^{2}+Q_{2}^{2}+P_{1}^{2}+P_{2}^{2}\right)}, \\
\rho_{8}=P_{2} \sqrt{2 h-\left(Q_{1}^{2}+Q_{2}^{2}+P_{1}^{2}+P_{2}^{2}\right)}, & \rho_{9}=Q_{1} P_{2}-Q_{2} P_{1} .
\end{array}
$$


A generalisation of these variables for a fully resonant Hamiltonian with $n$ degrees of freedom appears in [58] and in [57] for 2 degrees of freedom, deriving the construction of coordinates in full detail. Now, the coordinates $(Q, P)=\left(Q_{1}, Q_{2}, P_{1}, P_{2}\right)$ will be used in the analysis of the parametric stability of $\mathcal{O}_{C}^{1}$ and $\mathcal{O}_{C}^{2}$. The expressions of the equilibria in these coordinates are $\mathcal{O}_{C}^{1}=(0,0,0, \sqrt{h})$ and $\mathcal{O}_{C}^{2}=(0,0,0,-\sqrt{h})$ and Hamiltonian (34) reads as

$$
\begin{aligned}
\overline{\mathcal{H}}_{\epsilon}(Q, P)= & 2\left(d_{2}+d_{3}\right) h Q_{1}^{2}+2\left(d_{2}+d_{4}\right) h P_{1}^{2}+2 d_{5} h P_{2}^{2} \\
& -d_{3} Q_{1}^{4}-d_{4} P_{1}^{4}-\left(d_{3}+d_{4}\right) Q_{1}^{2} P_{1}^{2}-d_{5} P_{2}^{2}\left(Q_{2}^{2}+P_{2}^{2}\right) \\
& +\left(-d_{3}+d_{4}-d_{5}\right) Q_{1}^{2} P_{2}^{2}+\left(d_{3}-d_{4}-d_{5}\right) P_{1}^{2} P_{2}^{2} \\
& +2\left(d_{3}-d_{4}\right) Q_{1} Q_{2} P_{1} P_{2}+O\left(\epsilon^{2}\right) .
\end{aligned}
$$

The eigenvalues related to the linearisation of $\overline{\mathcal{H}}_{\epsilon}(Q, P)$ evaluated at $(0,0,0, \pm \sqrt{h})$ are $\pm i \alpha_{1}, \pm i \alpha_{2}$, with

$$
\alpha_{1}=2\left(2 d_{2}+d_{3}+d_{4}-d_{5}\right) h, \quad \alpha_{2}=4 d_{5} h .
$$

Then, for $\left(2 d_{2}+d_{3}+d_{4}-d_{5}\right) d_{5} \neq 0$ the equilibria are non-degenerate state points of centre type. Thus, by Reeb's Theorem [62], for a sufficiently small $\epsilon$, they give rise to families (parametrised by $h$ ) of periodic solutions of the system with Hamiltonian (6) whose projections in configuration space are close to (prograde and retrograde) circular trajectories on the $O x y$-plane.

The period is computed as $2 \pi\left|\partial \mathcal{H}_{\epsilon}(Q, P) / \partial h\right|^{-1}$, where $\mathcal{H}_{\epsilon}(Q, P)=h+\epsilon^{2}\left[4 d_{1} h^{2}\right.$ $\left.+\overline{\mathcal{H}}_{\epsilon}(Q, P)\right] / 2$, evaluating the result at $(Q, P)=\mathcal{O}_{C}^{j}$, expanding it in terms of $\epsilon$ around 0 and truncating at order 4 in $\epsilon$, see details in [71]. We get $T(\epsilon)=$ $2 \pi\left[1 \mp \epsilon^{2}\left|4 d_{1}+d_{5}\right| h\right]+O\left(\epsilon^{4}\right)$.

The characteristic multipliers are obtained from the eigenvalues associated to the linearisation, that is, from $\pm i \alpha_{1}, \pm i \alpha_{2}$, see for instance $[54,71]$. In particular, we get $1,1,1 \pm 2 \pi i \epsilon^{2} \alpha_{1}+O\left(\epsilon^{4}\right), 1 \pm 2 \pi i \epsilon^{2} \alpha_{2}+O\left(\epsilon^{4}\right)$.

Moreover, it is straightforward to check that $\mathcal{O}_{C}^{j}$ are in 1:-1 resonance if and only if $2 d_{2}+d_{3}+d_{4}-3 d_{5}=0$. Thus, if this condition is not fulfilled the equilibria 
$\mathcal{O}_{C}^{j}$ are parametrically stable and the periodic solutions reconstructed from these points are linearly stable.

Remark 2. The equilibria $\mathcal{O}_{C}^{j}$ in $\mathbb{C P}_{h}^{2}$ correspond to the equilibria $\mathcal{O}^{j}$ in $\mathcal{M}_{\gamma}$, as they are associated with the same periodic solutions for Hamiltonian (6). Moreover, the period calculated in Theorem 3.1 agrees with the period computed in Theorem 4.1 because in the case of circular-equatorial solutions $h=|N|=|\gamma|$. The characteristic multipliers of the periodic solutions have been naturally computed in the analysis made in $\mathbb{C P}_{h}^{2}$, because the way to obtain them from $\mathcal{M}_{\gamma}$ would be more involved. Nevertheless, the conditions on the parameters for the existence of the periodic solutions are more restrictive when performing the analysis from $\mathbb{C P}_{h}^{2}$ than when doing it from $\mathcal{M}_{\gamma}$.

Our second result in this section concerns the existence of periodic solutions of rectilinear type.

Theorem 4.2. When $\left(d_{3}-d_{2}\right)\left(d_{4}-d_{2}\right) \neq 0$ the point in $\mathbb{C P}_{h}^{2}$ with coordinates $\mathcal{O}_{R}=(0,2 h, 0,0,0,0,0,0,0)$ is an equilibrium of system (35) and, for a sufficiently small $\epsilon>0$, it gives rise to a family of periodic solutions of Hamiltonian (6) parametrised by $h>0$ whose projection in configuration space is close to rectilinear trajectories in the Oz-axis. Moreover, when $\left(d_{3}-d_{2}\right)\left(d_{4}-d_{2}\right)>0$ the point $\mathcal{O}_{R}$ is in 1:1 resonance and the corresponding periodic solutions are linearly stable whereas when $\left(d_{3}-d_{2}\right)\left(d_{4}-d_{2}\right)<0$ the point $\mathcal{O}_{R}$ is of saddle type and the associated periodic solutions are unstable. The characteristic multipliers of the periodic solutions are $1,1,1 \pm 8 \pi i \epsilon^{2} \sqrt{\left(d_{3}-d_{2}\right)\left(d_{4}-d_{2}\right)} h+O\left(\epsilon^{4}\right), 1 \pm 8 \pi i \epsilon^{2} \sqrt{\left(d_{3}-d_{2}\right)\left(d_{4}-d_{2}\right)} h+O\left(\epsilon^{4}\right)$ whereas their periods are $T(\epsilon)=2 \pi\left[1-4 \epsilon^{2}\left|d_{1}+d_{2}\right| h\right]+O\left(\epsilon^{4}\right)$.

Proof It is trivially checked that $\mathcal{O}_{R}$ is an isolated equilibrium of the vector field (35). From the expressions of the invariants in rectangular variables, formulae (30), it is easily inferred that $\mathcal{O}_{R}$ corresponds to a family of periodic solutions in $T^{*} \mathbb{R}^{3}$ whose projections in configuration space $O x y z$ are rectilinear solutions in the $O z$-axis. 
To study the parametric stability of $\mathcal{O}_{R}$ we should introduce adequate local symplectic coordinates. In this case we cannot use the set (37), that was valid for the circular-equatorial solutions, because now $\rho_{1}=0$. So, following a similar approach we have found a set of coordinates that works properly for this kind of solutions. Concretely we introduce

$$
Q_{1}=\frac{\rho_{6}}{\sqrt{\rho_{2}}}, \quad Q_{2}=\frac{\rho_{4}}{\sqrt{\rho_{2}}}, \quad P_{1}=\frac{\rho_{9}}{\sqrt{\rho_{2}}}, \quad P_{2}=-\frac{\rho_{7}}{\sqrt{\rho_{2}}},
$$

that is not defined for $\rho_{2}=0$. Note however that in this case we have $\rho_{2}=2 h \neq 0$. The inverse transformation is

$$
\begin{array}{cl}
\rho_{1}=Q_{2}^{2}+P_{2}^{2}, \quad \rho_{2}=2 h-\left(Q_{1}^{2}+Q_{2}^{2}+P_{1}^{2}+P_{2}^{2}\right), \quad \rho_{3}=Q_{1}^{2}+P_{1}^{2}, \\
\rho_{4}=Q_{2} \sqrt{2 h-\left(Q_{1}^{2}+Q_{2}^{2}+P_{1}^{2}+P_{2}^{2}\right)}, & \rho_{5}=Q_{1} Q_{2}+P_{1} P_{2}, \\
\rho_{6}=Q_{1} \sqrt{2 h-\left(Q_{1}^{2}+Q_{2}^{2}+P_{1}^{2}+P_{2}^{2}\right)}, & \rho_{7}=-P_{2} \sqrt{2 h-\left(Q_{1}^{2}+Q_{2}^{2}+P_{1}^{2}+P_{2}^{2}\right)}, \\
\rho_{8}=Q_{2} P_{1}-Q_{1} P_{2}, & \rho_{9}=P_{1} \sqrt{2 h-\left(Q_{1}^{2}+Q_{2}^{2}+P_{1}^{2}+P_{2}^{2}\right)} .
\end{array}
$$

By construction the equilibrium $\mathcal{O}_{R}$ has coordinates $(0,0,0,0)$ and Hamiltonian (34) assumes the form

$$
\begin{aligned}
\overline{\mathcal{H}}_{\epsilon}(Q, P)= & 4 d_{2} h^{2}+2 \omega_{1} h\left(Q_{1}^{2}+Q_{2}^{2}\right)+2 \omega_{2} h\left(P_{1}^{2}+P_{2}^{2}\right) \\
& -d_{3}\left(Q_{1}^{2}+Q_{2}^{2}\right)^{2}-d_{4}\left(P_{1}^{2}+P_{2}^{2}\right)^{2}-\left(d_{3}+d_{4}\right)\left(Q_{1}^{2} P_{1}^{2}+Q_{2}^{2} P_{2}^{2}\right) \\
& -\left(d_{3}+d_{4}-d_{5}\right)\left(Q_{1}^{2} P_{2}^{2}+Q_{2}^{2} P_{1}^{2}\right)-2 d_{5} Q_{1} Q_{2} P_{1} P_{2}+O\left(\epsilon^{2}\right),
\end{aligned}
$$

with $\omega_{1}, \omega_{2}$ defined as

$$
\omega_{1}=d_{3}-d_{2}, \quad \omega_{2}=d_{4}-d_{2}
$$

The eigenvalues related to the linearisation of $\overline{\mathcal{H}}_{\epsilon}(Q, P)$ evaluated at $(0,0,0,0)$ are $\pm 4 i \sqrt{\omega_{1} \omega_{2}} h$ with multiplicity two and the corresponding eigenvectors form a basis of $\mathbb{R}^{4}$. Hence, when $\omega_{1} \omega_{2} \neq 0$ the equilibrium point $\mathcal{O}_{R}$ is non-degenerate and by Reeb's Theorem [62], Hamiltonian (6) has one family (parametrised by $h$ ) of periodic solutions in $T^{*} \mathbb{R}^{3}$ whose projections in configuration space $O x y z$ are near rectilinear trajectories in the $O z$-axis. When $\omega_{1} \omega_{2}>0$, the equilibrium is stable of 
centre type and moreover it is in 1:1 resonance, therefore $\mathcal{O}_{R}$ is a parametrically stable point and the periodic solutions reconstructed from $\mathcal{O}_{R}$ are linearly stable. On the other hand for $\omega_{1} \omega_{2}<0$, the point $\mathcal{O}_{R}$ is unstable with linearisation saddle $\times$ saddle.

Proceeding as in the proof of Theorem 4.1 for the computation of the period of the periodic solutions, we get $T(\epsilon)=2 \pi\left[1-4 \epsilon^{2}\left|d_{1}+d_{2}\right| h\right]+O\left(\epsilon^{4}\right)$. Finally, the characteristic multipliers of the periodic solutions are obtained from the eigenvalues of the linearisation of $\overline{\mathcal{H}}_{\epsilon}(Q, P)$ around the origin, yielding $1,1,1 \pm 8 \pi i \epsilon^{2} \sqrt{\omega_{1} \omega_{2}} h+$ $O\left(\epsilon^{4}\right), 1 \pm 8 \pi i \epsilon^{2} \sqrt{\omega_{1} \omega_{2}} h+O\left(\epsilon^{4}\right)$.

Remark 3. The change of linear stability observed in Theorem 4.2 will be analysed in detail in Section 5, concretely in Theorem 5.5 where we will deal with bifurcations of the singular points related to rectilinear motions on the OzZ-plane. Specifically, we will prove that a Hamiltonian Hopf bifurcation takes place for this kind of motions and for some relations of the parameters $a_{i}, b_{j}$.

4.3. KAM tori of dimension 3 reconstructed from $\mathbb{C P}_{h}^{2}$. In this subsection we prove the existence of KAM 3-tori associated to the equilibria $\mathcal{O}_{C}^{1}, \mathcal{O}_{C}^{2}$ and $\mathcal{O}_{R}$. We start with the tori related to the circular-equatorial solutions.

Theorem 4.3. When $\left(2 d_{2}+d_{3}+d_{4}-d_{5}\right) d_{5} \neq 0$ and $2 d_{2}+d_{3}+d_{4} \neq 0$, and for $a$ sufficiently small $\epsilon>0$, there are invariant KAM 3-tori for Hamiltonian system (6) around the nearly circular-equatorial periodic solutions. These invariant tori form a majority in the sense that the measure of the complement of their union is of order $O\left(\epsilon^{\mu / 2}\right)$ for a pre-fixed constant $0<\mu<1 / 5$.

Proof Going back to Hamiltonian (38), we need to shift the origin to the equilibrium $\mathcal{O}_{C}^{j}$ and rescale conveniently to make the ordering of the terms in $\overline{\mathcal{H}}_{\epsilon}$ explicit. For that, we apply the change

$$
Q_{1}=\epsilon^{1 / 4} \bar{Q}_{1}, \quad Q_{2}=\epsilon^{1 / 4} \bar{Q}_{2}, \quad P_{1}=\epsilon^{1 / 4} \bar{P}_{1}, \quad P_{2}=\epsilon^{1 / 4} \bar{P}_{2} \pm \sqrt{h},
$$


where the upper sign goes with $\mathcal{O}_{C}^{1}$ whereas the lower sign goes with $\mathcal{O}_{C}^{2}$. The transformation is symplectic with multiplier $\epsilon^{-1 / 2}$. After expanding in powers of $\epsilon$, Hamiltonian (38) is transformed into

$$
\overline{\mathcal{H}}_{\epsilon}(\bar{Q}, \bar{P})=\overline{\mathcal{H}}_{\epsilon, 0}+\overline{\mathcal{H}}_{2}+\epsilon^{1 / 4} \overline{\mathcal{H}}_{3}+\epsilon^{1 / 2} \overline{\mathcal{H}}_{4}+O\left(\epsilon^{3 / 2}\right)
$$

where $\overline{\mathcal{H}}_{\epsilon, 0}=\epsilon^{-1 / 2} d_{5} h^{2}$

$$
\overline{\mathcal{H}}_{2}=\left(2 d_{2}+d_{3}+d_{4}-d_{5}\right) h\left(\bar{Q}_{1}^{2}+\bar{P}_{1}^{2}\right)-d_{5} h\left(\bar{Q}_{2}^{2}+4 \bar{P}_{2}^{2}\right)
$$

whereas $\overline{\mathcal{H}}_{3}$ and $\overline{\mathcal{H}}_{4}$ contain terms of degree 3 and 4 , respectively, in $(\bar{Q}, \bar{P})$.

With the aim of diagonalising the quadratic part in $(\bar{Q}, \bar{P})$ of Hamiltonian (43), we apply a complex symplectic change $\left(\bar{Q}_{j}, \bar{P}_{j}\right) \rightarrow\left(q_{j}, p_{j}\right)$, given explicitly by

$$
\bar{Q}_{1}=\frac{1-i}{2}\left(q_{1}-p_{1}\right), \bar{Q}_{2}=\frac{1+i}{\sqrt{2}}\left(q_{2}-p_{2}\right), \bar{P}_{1}=\frac{1+i}{2}\left(q_{1}+p_{1}\right), \quad \bar{P}_{2}=\frac{1-i}{2 \sqrt{2}}\left(q_{2}+p_{2}\right)
$$

Then $\overline{\mathcal{H}}_{2}$ is converted into $i\left(\alpha_{1} q_{1} p_{1}+\alpha_{2} q_{2} p_{2}\right)$, where $\alpha_{1} \neq 0$ and $\alpha_{2} \neq 0$ are given in (39). Higher order terms are transformed accordingly.

Next, we normalise Hamiltonian $\overline{\mathcal{H}}_{\epsilon}$ including terms of degree 4 in $(q, p)$ obtaining

$$
\overline{\mathcal{H}}_{\epsilon}\left(q_{1}, q_{2}, p_{1}, p_{2}\right)=\overline{\mathcal{H}}_{\epsilon, 0}+i\left(\alpha_{1} q_{1} p_{1}+\alpha_{2} q_{2} p_{2}\right)+\epsilon^{1 / 2} \overline{\mathcal{H}}_{4}+O\left(\epsilon^{3 / 2}\right)
$$

and $\overline{\mathcal{H}}_{4}$ contains terms of degree 4 in $\left(q_{1}, q_{2}, p_{1}, p_{2}\right)$ that are in normal form with respect to the quadratic terms. The transformation is not defined for $2 d_{2}+d_{3}+d_{4}=$ 0 because this term appears in the denominators of the generating function. Indeed when $2 d_{2}+d_{3}+d_{4}=0$ the points $\mathcal{O}_{C}^{j}$ are in 2:-1 resonance.

At this point we should prepare the Hamiltonian to apply a KAM-type theorem. For achieving it, we introduce adequate action-angle variables $(I, \phi)=$ $\left(I_{1}, I_{2}, \phi_{1}, \phi_{2}\right)$ defined by the relations

$$
\begin{array}{ll}
q_{1}=\sqrt{I_{1}}\left(\cos \phi_{1}-i \sin \phi_{1}\right), & q_{2}=\sqrt{I_{2}}\left(\cos \phi_{2}-i \sin \phi_{2}\right), \\
p_{1}=\sqrt{I_{1}}\left(\sin \phi_{1}-i \cos \phi_{1}\right), & p_{2}=\sqrt{I_{2}}\left(\sin \phi_{2}-i \cos \phi_{2}\right) .
\end{array}
$$


Now, recall that the full Hamiltonian is $\mathcal{H}_{\epsilon}$ given in (32). Then, by subtracting $h$, multiplying by $2 / \epsilon^{2}$ and subtracting $4 d_{1} h^{2}$ we arrived at $\overline{\mathcal{H}}_{\epsilon}$ given in (34). Then, we divided by $\epsilon^{1 / 2}$ to obtain $\overline{\mathcal{H}}_{\epsilon}$, see (43). So, we should undo these operations at once. We identify $h$ with the action $J_{1}$, that has been already defined at the beginning of the proof of Theorem 4.1. Then, we rescale time by multiplying the resulting Hamiltonian by $\epsilon^{1 / 2}$, add $4 d_{1} J_{1}^{2}$, rescale time again by multiplying the Hamiltonian by $\epsilon^{2} / 2$ and add $J_{1}$. We end up with

$$
\mathcal{H}_{\epsilon}\left(\phi, \theta_{1}, I, J_{1}\right)=h_{0}\left(J_{1}\right)+\eta^{4} h_{1}\left(J_{1}\right)+\eta^{5} h_{2}\left(J_{1}, I_{1}, I_{2}\right)+\eta^{6} h_{3}\left(J_{1}, I_{1}, I_{2}\right)+O\left(\eta^{8}\right),
$$

where $\eta=\epsilon^{1 / 2}$ and

$$
\begin{gathered}
h_{0}=J_{1}, \quad h_{1}=\frac{1}{2}\left(4 d_{1}+d_{5}\right) J_{1}^{2}, \quad h_{2}=\frac{1}{2}\left(\alpha_{1} I_{1}+\alpha_{2} I_{2}\right), \\
h_{3}=\Delta_{2} I_{1}^{2}+\Delta_{1} I_{1} I_{2}+2 d_{5} I_{2}^{2},
\end{gathered}
$$

with

$$
\begin{aligned}
& \Delta_{1}=\frac{2\left(d_{3}-d_{4}\right)^{2}}{2 d_{2}+d_{3}+d_{4}}-2 d_{5} \\
& \Delta_{2}=-\frac{d_{3}^{2}+d_{3}\left(6 d_{4}-d_{5}\right)+d_{4}\left(d_{4}-d_{5}\right)+2 d_{2}\left[2\left(d_{3}+d_{4}\right)-d_{5}\right]}{2\left(2 d_{2}+d_{3}+d_{4}\right)}
\end{aligned}
$$

Now, we are in a position to apply the Theorem by Han, Li and Yi [39]. By using the notation in [39] we get $n=3, a=3, m_{1}=4, m_{2}=5, m_{3}=6$, $n_{0}=n_{1}=1, n_{2}=n_{3}=3, y^{n_{0}}=\hat{y}^{n_{0}}=y^{n_{1}}=\hat{y}^{n_{1}}=J_{1}, y^{n_{2}}=y^{n_{3}}=\left(J_{1}, I_{1}, I_{2}\right)$, $\hat{y}^{n_{2}}=\hat{y}^{n_{3}}=\left(I_{1}, I_{2}\right)$. The vector of frequencies has dimension 6 and is given by

$$
\Omega\left(J_{1}, I_{1}, I_{2}\right)=\left(\frac{\partial h_{0}}{\partial J_{1}}, \frac{\partial h_{1}}{\partial J_{1}}, \frac{\partial h_{2}}{\partial I_{1}}, \frac{\partial h_{2}}{\partial I_{2}}, \frac{\partial h_{3}}{\partial I_{1}}, \frac{\partial h_{3}}{\partial I_{2}}\right)
$$


and the $6 \times 4$ matrix whose columns are $\Omega, \partial \Omega / \partial J_{1}, \partial \Omega / \partial I_{1}$ and $\partial \Omega / \partial I_{2}$ reads as

$$
\left[\begin{array}{cccc}
\Omega_{1} & \frac{\partial \Omega_{1}}{\partial J_{1}} & \frac{\partial \Omega_{1}}{\partial I_{1}} & \frac{\partial \Omega_{1}}{\partial I_{2}} \\
\Omega_{2} & \frac{\partial \Omega_{2}}{\partial J_{1}} & \frac{\partial \Omega_{2}}{\partial I_{1}} & \frac{\partial \Omega_{2}}{\partial I_{2}} \\
\Omega_{3} & \frac{\partial \Omega_{3}}{\partial J_{1}} & \frac{\partial \Omega_{3}}{\partial I_{1}} & \frac{\partial \Omega_{3}}{\partial I_{2}} \\
\Omega_{4} & \frac{\partial \Omega_{4}}{\partial J_{1}} & \frac{\partial \Omega_{4}}{\partial I_{1}} & \frac{\partial \Omega_{4}}{\partial I_{2}} \\
\Omega_{5} & \frac{\partial \Omega_{5}}{\partial J_{1}} & \frac{\partial \Omega_{5}}{\partial I_{1}} & \frac{\partial \Omega_{5}}{\partial I_{2}} \\
\Omega_{6} & \frac{\partial \Omega_{6}}{\partial J_{1}} & \frac{\partial \Omega_{6}}{\partial I_{1}} & \frac{\partial \Omega_{6}}{\partial I_{2}}
\end{array}\right]=\left[\begin{array}{cccc}
0 & 0 & 0 \\
\frac{\alpha_{1}}{2} & \frac{\alpha_{1}}{2 J_{1}} & 0 & 0 \\
\frac{\alpha_{2}}{2} & \frac{\alpha_{2}}{2 J_{1}} & 0 & 0 \\
2 \Delta_{2} I_{1}+\Delta_{1} I_{2} & 0 & 2 d_{2} & \Delta_{1} \\
\Delta_{1} I_{1}+\frac{\alpha_{2}}{J_{1}} I_{2} & 0 & \Delta_{1} & \frac{\alpha_{2}}{J_{1}}
\end{array}\right]
$$

Taking into account that $d_{5} \neq 0$, this matrix has rank $\geq 3=n$. Note that conditions $2 d_{2}+d_{3}+d_{4}-d_{5} \neq 0$ and $2 d_{2}+d_{3}+d_{4} \neq 0$ are also needed. Then, Han-Li-Yi's Theorem applies and guarantees the existence of KAM tori of dimension 3 around the circular-equatorial periodic solutions. Setting $b=\sum_{j=1}^{a} m_{j}\left(n_{j}-n_{j-1}\right)$, we obtain $b=10$. The maximum order of the partial derivatives involved in the computation of the $6 \times 4$ matrix is $s=1$. As the perturbation is of order $O\left(\eta^{8}\right)$ and $\eta^{s b+\mu}=\eta^{10+\mu}<\eta^{8}$, the excluding measure for the existence of the quasi-periodic invariant tori is of order $\eta^{\mu / s}=\eta^{\mu}=\epsilon^{\mu / 2}$ for a pre-fixed small positive constant $\mu<1 / 5$.

Remark 4. We stress that the constant $m$ introduced in (25) is related to the $d_{i}$ by $m=6\left(2 d_{2}+d_{3}+d_{4}\right)$ whereas the other conditions on Theorems 3.2 and 4.3 are independent. We also emphasise that the excluding measure for the existence of the quasi-periodic invariant tori is smaller for the KAM tori reconstructed from $\mathcal{M}_{\gamma}$.

Now we deal with the invariant tori associated to rectilinear motions in the $O z Z$-plane.

For $\left(d_{3}-d_{2}\right)\left(d_{4}-d_{2}\right) \neq 0$ we define

$$
\Delta=\frac{d_{5}}{2}+\frac{d_{2}\left(d_{3}-d_{4}\right)^{2}}{4\left(d_{3}-d_{2}\right)\left(d_{4}-d_{2}\right)} .
$$


Theorem 4.4. When $\left(d_{3}-d_{2}\right)\left(d_{4}-d_{2}\right)>0, \Delta \neq 0$ and, for a sufficiently small $\epsilon>0$, there are invariant KAM 3-tori for Hamiltonian system (6) around the periodic solutions whose projections in configuration space Oxyz are nearly rectilinear trajectories in the Oz-axis. These invariant tori form a majority in the sense that the measure of the complement of their union is of order $O\left(\epsilon^{\mu / 2}\right)$ for a pre-fixed constant $0<\mu<1 / 5$.

Proof Starting with Hamiltonian (41), we rescale coordinates and time adequately to make the ordering of the terms in $\overline{\mathcal{H}}_{\epsilon}$ explicit. For that, we divide $\overline{\mathcal{H}}_{\epsilon}$ by $\epsilon^{1 / 2}$ and apply the transformation $Q_{j}=\epsilon^{1 / 4} \bar{Q}_{j}, P_{j}=\epsilon^{1 / 4} \bar{P}_{j}$ for $j=1,2$. Using this change Hamiltonian (41) gets transformed into

$$
\overline{\mathcal{H}}_{\epsilon}(\bar{Q}, \bar{P})=\overline{\mathcal{H}}_{\epsilon, 0}+\overline{\mathcal{H}}_{2}+\epsilon^{1 / 2} \overline{\mathcal{H}}_{4}+O\left(\epsilon^{3 / 2}\right),
$$

where $\overline{\mathcal{H}}_{\epsilon, 0}=4 \epsilon^{-1 / 2} d_{2} h^{2}$,

$$
\overline{\mathcal{H}}_{2}=2 \omega_{1} h\left(\bar{Q}_{1}^{2}+\bar{Q}_{2}^{2}\right)+2 \omega_{2} h\left(\bar{P}_{1}^{2}+\bar{P}_{2}^{2}\right)
$$

and $\overline{\mathcal{H}}_{4}$ contains terms of degree 4 in $(\bar{Q}, \bar{P})$ and is independent of $\epsilon$.

The next step is the diagonalisation of $\overline{\mathcal{H}}_{2}$. For this aim we construct a symplectic transformation $\left(\bar{Q}_{1}, \bar{Q}_{2}, \bar{P}_{1}, \bar{P}_{2}\right) \longrightarrow\left(q_{1}, q_{2}, p_{1}, p_{2}\right)$ by means of the eigenvectors attached to $\overline{\mathcal{H}}_{2}$, see $[45,50]$. This transformation is given by

$$
\begin{array}{ll}
\bar{Q}_{1}=\frac{1-i}{2}\left(\frac{\omega_{2}}{\omega_{1}}\right)^{1 / 4}\left(q_{2}-p_{2}\right), & \bar{Q}_{2}=\frac{1-i}{2}\left(\frac{\omega_{2}}{\omega_{1}}\right)^{1 / 4}\left(q_{1}-p_{1}\right), \\
\bar{P}_{1}=\frac{1+i}{2}\left(\frac{\omega_{1}}{\omega_{2}}\right)^{1 / 4}\left(q_{2}+p_{2}\right), & \bar{P}_{2}=\frac{1+i}{2}\left(\frac{\omega_{1}}{\omega_{2}}\right)^{1 / 4}\left(q_{1}+p_{1}\right) .
\end{array}
$$

Note that $\omega_{1} \omega_{2}>0$ because the equilibrium is a linear centre. Applying this change of variables to (47) and normalising up to the inclusion of terms of degree 4 in $(q, p)$ by means of the Lie-Deprit process [18], we arrive at the Hamiltonian

$$
\overline{\mathcal{H}}_{\epsilon}(q, p)=\overline{\mathcal{H}}_{\epsilon, 0}+4 i \sqrt{\omega_{1} \omega_{2}} h\left(q_{1} p_{1}+q_{2} p_{2}\right)+\epsilon^{1 / 2} \overline{\mathcal{H}}_{4}+O\left(\epsilon^{3 / 2}\right)
$$


where $\overline{\mathcal{H}}_{4}$ contains terms of degree 4 in $(q, p)$ that are in normal form with respect to the quadratic part of $\overline{\mathcal{H}}_{\epsilon}(q, p)$. The transformation is not defined for $\omega_{1} \omega_{2}=0$, but we are assuming $\omega_{1} \omega_{2}>0$.

At this point we prepare the normalised Hamiltonian to apply Han-Li-Yi's Theorem. For that, we should reverse the operations that passed from $\mathcal{H}_{\epsilon}$ to $\overline{\mathcal{H}}_{\epsilon}$ and express the Hamiltonian in action-angle coordinates. Thus, we identify $h$ with $J_{1}$, then rescale time by multiplying the resulting Hamiltonian by $\epsilon^{1 / 2}$, add $4 d_{1} J_{1}^{2}$, rescale time again by multiplying the Hamiltonian by $\epsilon^{2} / 2$ and add $J_{1}$. Due to the fact that $\overline{\mathcal{H}}_{2}$ is in 1:1 resonance, we choose planar Lissajous coordinates [19] in order to express $\mathcal{H}_{\epsilon}$ conveniently. Specifically, this set of variables allows us to eliminate the dependence of the angles in the Hamiltonian up to $O\left(\epsilon^{3 / 2}\right)$. First, we put $\left(q_{1}, q_{2}, p_{1}, p_{2}\right)$ in rectangular coordinates $\left(x_{1}, x_{2}, X_{1}, X_{2}\right)$ as

$q_{1}=\frac{1}{\sqrt{2}}\left(x_{1}-i X_{1}\right), q_{2}=\frac{1}{\sqrt{2}}\left(x_{2}-i X_{2}\right), p_{1}=\frac{1}{\sqrt{2}}\left(X_{1}-i x_{1}\right), p_{2}=\frac{1}{\sqrt{2}}\left(X_{2}-i x_{2}\right)$.

Now we apply the Lissajous transformation through the symplectic change:

$$
\begin{aligned}
& x_{1}=\sqrt{\frac{1}{2}\left(L_{1}+L_{2}\right)} \cos \left(\ell_{1}+\ell_{2}\right)-\sqrt{\frac{1}{2}\left(L_{1}-L_{2}\right)} \cos \left(\ell_{1}-\ell_{2}\right), \\
& x_{2}=\sqrt{\frac{1}{2}\left(L_{1}+L_{2}\right)} \sin \left(\ell_{1}+\ell_{2}\right)+\sqrt{\frac{1}{2}\left(L_{1}-L_{2}\right)} \sin \left(\ell_{1}-\ell_{2}\right), \\
& X_{1}=-\sqrt{\frac{1}{2}\left(L_{1}+L_{2}\right)} \sin \left(\ell_{1}+\ell_{2}\right)+\sqrt{\frac{1}{2}\left(L_{1}-L_{2}\right)} \sin \left(\ell_{1}-\ell_{2}\right), \\
& X_{2}=\sqrt{\frac{1}{2}\left(L_{1}+L_{2}\right)} \cos \left(\ell_{1}+\ell_{2}\right)+\sqrt{\frac{1}{2}\left(L_{1}-L_{2}\right)} \cos \left(\ell_{1}-\ell_{2}\right) .
\end{aligned}
$$

The interesting feature of this transformation in our setting is that the action $L_{2}$ is proportional to $N$, in fact $N=\epsilon^{1 / 2} L_{2}$. Thence, since the whole system is axially symmetric, Hamiltonian $\mathcal{H}_{\epsilon}$ written in Lissajous coordinates is independent of $\ell_{2}$ and has $L_{2}$ as an integral, thus one can use $L_{2}$ as one of the actions needed in the application of Han-Li-Yi's Theorem. Moreover the normalisation performed before leading to Hamiltonian (48) has the effect of making $\mathcal{H}_{\epsilon}$, after transforming it into Lissajous coordinates, independent of $\ell_{1}$ at least up to terms of order $O\left(\epsilon^{4}\right)$. After 
some simplifications, we end up with the Hamiltonian

$$
\begin{aligned}
\mathcal{H}_{\epsilon}\left(\ell_{1}, \theta_{1}, L_{1}, L_{2}, J_{1}\right)= & h_{0}\left(J_{1}\right)+\eta^{4} h_{1}\left(J_{1}\right)+\eta^{5} h_{2}\left(J_{1}, L_{1}\right)+\eta^{6} h_{3}\left(J_{1}, L_{1}, L_{2}\right) \\
& +O\left(\eta^{8}\right)
\end{aligned}
$$

where

$$
\begin{aligned}
& h_{0}=J_{1}, \quad h_{1}=2\left(d_{1}+d_{2}\right) J_{1}^{2}, \quad h_{2}=2 \sqrt{\omega_{1} \omega_{2}} J_{1} L_{1}, \\
& h_{3}=-\left[\omega_{1}+\omega_{2}+d_{2}\left(\frac{1}{2}+\frac{3 \omega_{1}}{4 \omega_{2}}+\frac{3 \omega_{2}}{4 \omega_{1}}\right)\right] L_{1}^{2}+\Delta L_{2}^{2},
\end{aligned}
$$

with $\eta=\epsilon^{1 / 2}$ and $\Delta$ given in (46). In the notation of Han-Li-Yi's Theorem, we have $n=3, a=3, m_{1}=4, m_{2}=5, m_{3}=6, n_{0}=n_{1}=1, n_{2}=2, n_{3}=3$, $y^{n_{0}}=\hat{y}^{n_{0}}=y^{n_{1}}=\hat{y}^{n_{1}}=J_{1}, y^{n_{2}}=\left(J_{1}, L_{1}\right), y^{n_{3}}=\left(J_{1}, L_{1}, L_{2}\right), \hat{y}^{n_{2}}=L_{1}$, $\hat{y}^{n_{3}}=L_{2}$ and the 4 -dimensional vector of frequencies is given by

$$
\Omega\left(J_{1}, L_{1}, L_{2}\right)=\left(\frac{\partial h_{0}}{\partial J_{1}}, \frac{\partial h_{1}}{\partial J_{1}}, \frac{\partial h_{2}}{\partial L_{1}}, \frac{\partial h_{3}}{\partial L_{2}}\right)
$$

The $4 \times 4$ matrix whose columns are $\Omega, \partial \Omega / \partial J_{1}, \partial \Omega / \partial L_{1}$ and $\partial \Omega / \partial L_{2}$ reads as

$$
\left[\begin{array}{cccc}
\Omega_{1} & \frac{\partial \Omega_{1}}{\partial J_{1}} & \frac{\partial \Omega_{1}}{\partial L_{1}} & \frac{\partial \Omega_{1}}{\partial L_{2}} \\
\Omega_{2} & \frac{\partial \Omega_{2}}{\partial J_{1}} & \frac{\partial \Omega_{2}}{\partial L_{1}} & \frac{\partial \Omega_{2}}{\partial L_{2}} \\
\Omega_{3} & \frac{\partial \Omega_{3}}{\partial J_{1}} & \frac{\partial \Omega_{3}}{\partial L_{1}} & \frac{\partial \Omega_{3}}{\partial L_{2}} \\
\Omega_{4} & \frac{\partial \Omega_{4}}{\partial J_{1}} & \frac{\partial \Omega_{4}}{\partial L_{1}} & \frac{\partial \Omega_{4}}{\partial L_{2}}
\end{array}\right]=\left[\begin{array}{cccc}
1 & 0 & 0 & 0 \\
4\left(d_{1}+d_{2}\right) J_{1} & 4\left(d_{1}+d_{2}\right) & 0 & 0 \\
2 \sqrt{\omega_{1} \omega_{2}} J_{1} & 2 \sqrt{\omega_{1} \omega_{2}} & 0 & 0 \\
2 \Delta L_{2} & 0 & 0 & 2 \Delta
\end{array}\right] .
$$

This matrix has rank 3 when $\Delta \neq 0$, thus Han-Li-Yi's Theorem guarantees the existence of KAM tori of dimension 3 around the periodic solutions whose projections in configuration space are of rectilinear type. In this case $b=\sum_{j=1}^{a} m_{j}\left(n_{j}-n_{j-1}\right)=11$ and the maximum order of the partial derivatives involved in the computation of the $4 \times 4$ matrix is $s=1$. As the perturbation is of order $O\left(\eta^{8}\right)$ and $\eta^{s b+\mu}=\eta^{11+\mu}<\eta^{8}$, for a pre-fixed small positive constant $\mu<1 / 5$, the excluding measure for the existence of the quasi-periodic invariant tori is of order $\eta^{\mu / s}=\eta^{\mu}=\epsilon^{\mu / 2}$ for $0<\mu<1 / 5$. 


\section{SECOND REDUCTION}

5.1. Reduction by the axial symmetry. In order to get the twice reduced space, first we have passed from $T^{*} \mathbb{R}^{3}$ to $\mathcal{M}_{\gamma}$ by means of the axial symmetry, and then reduce by the oscillator symmetry or, alternatively, reduce from $T^{*} \mathbb{R}^{3}$ to $\mathbb{C P}_{h}^{2}$ and as a second step reduce the axial symmetry.

If we choose the first approach we take into account that the space $\mathcal{M}_{\gamma}$ is described by the invariants of the axial symmetry, that is, the $i_{k}$ and its relations or constraints (8). The normalisation carried out in Section 4 together with the truncation of higher order terms allow us to make the resulting Hamiltonian system symmetric with respect to the flow generated by $\mathcal{H}_{0}$ in $(3)$, that is, the oscillator symmetry. This symmetry is the responsible of the second reduction. For achieving this second reduction we find the combinations of the invariants $\rho_{j}$ that remain invariants under rotations with respect to the $O z$-axis. From these combinations one obtains the invariants of the second reduction. After that, the reduced space, that we denote by $\mathcal{T}_{L, N}$ (where $L=h$ ), has dimension 2 in the 3 -dimensional space defined by three of these new invariants that we choose as the main invariants.

Proposition 5.1. The twice reduced space $\mathcal{T}_{L, N}$ is described by the three invariants

$$
\tau_{1}=\rho_{4}^{2}+\rho_{6}^{2}-\rho_{7}^{2}-\rho_{9}^{2}, \quad \tau_{2}=\rho_{1}+\rho_{3}, \quad \tau_{3}=2\left(\rho_{4} \rho_{7}-\rho_{6} \rho_{9}\right)
$$

with the restrictions $2|N| \leq \tau_{2} \leq 2 L$ and

$$
\tau_{1}^{2}+\tau_{3}^{2}=\left(2 L-\tau_{2}\right)^{2}\left(\tau_{2}^{2}-4 N^{2}\right)
$$

for every value of $L$ and $N$ such that $0 \leq|N|<L$.

Proof First we will find which of the polynomials $\rho_{j}$, or combinations of them, given in (7) remain invariant under the axial symmetry, that is, which of them can be written as combinations of the polynomials $i_{j}$ defined in (30). The fundamental 
relations between the invariants $\rho_{j}$ and $i_{k}$ are the following:

$$
\begin{gathered}
\rho_{2}=i_{5}^{2}+i_{6}^{2}, \quad \rho_{8}=i_{3}, \quad \rho_{1}+\rho_{3}=i_{1}+i_{2}, \quad \rho_{4}^{2}+\rho_{6}^{2}=i_{1} i_{5}^{2}+2 i_{4} i_{5} i_{6}+i_{2} i_{6}^{2} \\
\rho_{4}^{2}+\rho_{6}^{2}-\rho_{7}^{2}-\rho_{9}^{2}=\left(i_{1}-i_{2}\right)\left(i_{5}^{2}-i_{6}^{2}\right)+4 i_{4} i_{5} i_{6} \\
\rho_{4} \rho_{7}-\rho_{6} \rho_{9}=i_{5} i_{6}\left(i_{1}-i_{2}\right)-i_{4}\left(i_{5}^{2}-i_{6}^{2}\right) .
\end{gathered}
$$

Through the previous expressions we introduce the polynomials $\tau_{j}$ in terms of $\rho_{j}$ as follows

$$
\begin{array}{lll}
\tau_{1}=\rho_{4}^{2}+\rho_{6}^{2}-\rho_{7}^{2}-\rho_{9}^{2}, & \tau_{2}=\rho_{1}+\rho_{3}, & \tau_{3}=2\left(\rho_{4} \rho_{7}-\rho_{6} \rho_{9}\right), \\
\tau_{4}=\rho_{8}, & \tau_{5}=\rho_{2}, & \tau_{6}=\rho_{4}^{2}+\rho_{6}^{2},
\end{array}
$$

noting that these combinations of the $\rho_{j}$ remain invariant under the axial symmetry. Taking into account that $\rho_{j} \geq 0$ for $j=1,2,3$, it follows that

$$
\tau_{2}+\tau_{5}=2 L=2 h .
$$

Thus, we get the upper bound $\tau_{2} \leq 2 L$. From (52) it is deduced that

$$
2 \tau_{6}=\tau_{1}+\tau_{2} \tau_{5}
$$

and

$$
\tau_{1}^{2}+\tau_{3}^{2}=\tau_{5}^{2}\left(\tau_{2}^{2}-4 \tau_{4}^{2}\right)
$$

Using (53) and observing that $\tau_{4}=N$, it follows from (55) the relation (51). Also, since the left hand side of the equation (55) is non-negative, we must have that $2|N| \leq \tau_{2}$

The algebra of the new invariants is determined by the Poisson structure. Expressing the invariants $\tau_{j}$ as functions of $\rho_{j}$, computing the Poisson brackets in terms of them by making use of Table 2 and returning to the $\tau_{k}$, we end up with Table 3 . 


\begin{tabular}{|c||c|c|c|}
\hline$\left\{\tau_{j}, \tau_{k}\right\}$ & $\tau_{1}$ & $\tau_{2}$ & $\tau_{3}$ \\
\hline \hline$\tau_{1}$ & 0 & $-4 \tau_{3}$ & $8\left(\tau_{2}-2 L\right)\left[\left(L-\tau_{2}\right) \tau_{2}+2 N^{2}\right]$ \\
\hline$\tau_{2}$ & $4 \tau_{3}$ & 0 & $-4 \tau_{1}$ \\
\hline$\tau_{3}$ & $8\left(2 L-\tau_{2}\right)\left[\left(L-\tau_{2}\right) \tau_{2}+2 N^{2}\right]$ & $4 \tau_{1}$ & 0 \\
\hline
\end{tabular}

TABLE 3. Poisson brackets on the invariants $\tau_{i}$.

Remark 5. When $|N|=h=L$ the reduced space $\mathcal{T}_{L, \pm L}$ is merely a point, since then $\tau_{2}=L$ and $\tau_{1}=\tau_{3}=0$. It implies that the motions that are at the same time of circular and equatorial type cannot be studied in the twice reduced space. This is the reason of studying these type of solutions either in $\mathbb{C P}_{h}^{2}$ or in $\mathcal{M}_{\gamma}$.

Remark 6. The different symplectic reductions performed in the paper are sketched in the diagram of Fig. 1.

\section{Dimension Reductions and Spaces}

6

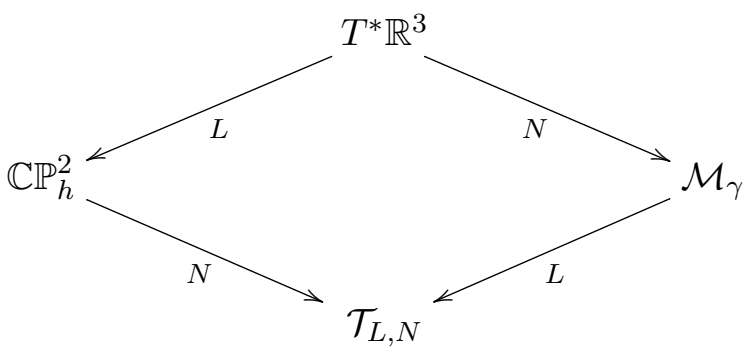

FIGURE 1. Scheme of reductions with the corresponding reduced spaces and integrals. The dimension of each space is shown in the left column.

5.2. Geometrical interpretation. Fixing the values $h$ and $\gamma$ of $L$ and $N$, the twice reduced space is defined by (51) together with the restriction $2|N| \leq \tau_{2}<2 L$. To study this space, we consider the auxiliary function $f: \mathbb{R}^{3} \rightarrow \mathbb{R}$ defined by

$$
f(\tau)=\tau_{1}^{2}+\tau_{3}^{2}-\left(2 L-\tau_{2}\right)^{2}\left(\tau_{2}^{2}-4 N^{2}\right),
$$

where $\tau=\left(\tau_{1}, \tau_{2}, \tau_{3}\right)$. By the implicit function theorem, the set defined by $f^{-1}(0)$ is regular in all the points where the gradient is non-null. In fact, $\nabla f(\tau)=$ 
$\left(2 \tau_{1},-4\left(2 L-\tau_{2}\right)\left[\left(L-\tau_{2}\right) \tau_{2}+2 N^{2}\right], 2 \tau_{3}\right)$ is null at the points: (i) $S_{1}=(0,2 L, 0)$ where $N \neq 0$; (ii) $S_{2}=(0,0,0)$ with $N=0$ and (iii) $S_{3}=(0,2 L, 0)$ also with $N=0$. When we fix $N \neq 0$ the twice reduced space $\mathcal{T}_{L, N}$ has one singularity corresponding to $S_{1}$. When we make $N=0$, there are two singularities that correspond to $S_{2}$ and $S_{3}$.

In order to get a geometrical representation of the twice reduced space, we fix the parameters $N$ and $L$, distinguishing two situations, either $N \neq 0$ or $N=0$.

In case (i) the twice reduced space is topologically a pinched sphere named balloon, or turnip, as it is labelled in [16] and [31]). In cases (ii) and (iii) it is a sphere with two singularities called lemon, see for example [16, 31].

At this point we introduce the set of action-angle coordinates devised for the 3-dimensional isotropic harmonic oscillator, the so called nodal-Lissajous variables $(\ell, g, \nu, L, G, N)$ proposed by Ferrer and Gárate [30] as a generalisation of the Lissajous coordinates of Deprit [19] for the 1:1:1 resonance, see also [70, 32, 33, 31]. The actions $L$ and $N$ are completed by the total angular momentum $G$ noticing that $|N| \leq G \leq L$. The conjugate angles, namely, $\ell, g$ and $\nu$ help to place the orbital ellipses of the oscillator in the 3-dimensional space. Specifically, the angle $\ell$ describes the position of the point $q$ on this ellipse in the $O x y z$-space, measured from the semi-minor axis; $g$ is the angle from the nodal line to this semi-minor axis and $\nu$ represents the ascending node of the orbital plane. Finally the inclination of the orbit is given by $\cos I=N / G$.

Our goal now is to understand which families of solutions are represented in each point of the twice reduced space for all possible values of $N$ and $L$. All families of solutions for the harmonic oscillator are represented in the balloons and lemons, excepting when $L=|N|$. We stress that in each balloon or lemon, all the families of trajectories have the same energy and the same third component of the angular momentum. We briefly describe the location of the most representative:

- Polar motions satisfy $N=0$ and are represented in the lemons. The rest of families are depicted in different balloons. 
- Rectilinear motions passing through the origin have null angular momentum vector. These motions correspond to a part of the lemon, represented by the generating curve of the lemon given by $\tau_{1}=\tau_{2}\left(2 L-\tau_{2}\right)$. The singularity $S_{2}$ corresponds to $\left(\tau_{1}, \tau_{2}, \tau_{3}\right)=(0,0,0)$, which accounts for the rectilinear trajectories with $x=y=X=Y=0$. The point $\tau_{2}=2 L$ represents the family of rectilinear-equatorial motions and it is the singularity $S_{3}$.

- Equatorial motions (prograde and retrograde) are those trajectories with $G=|N|$. In the invariants $\tau_{i}$ one has $\tau_{2}=2 L$ whereas $N$ takes any value in $[0, L)$. For $N=0$ we get rectilinear-equatorial trajectories and this case corresponds to $S_{3}$. On the other hand the point $(0,2 L, 0)$ corresponds to $S_{1}$ in the balloon.

- Circular motions satisfy $G=L$. It can be proved that circular motions in the reduced space $\mathcal{T}_{L, N}$ are represented either in the balloon or in the lemon by the point $\left(\tau_{1}, \tau_{2}, \tau_{3}\right)=\left(-2\left(L^{2}-N^{2}\right)^{2} / L^{2},\left(L^{2}+N^{2}\right) / L, 0\right)$, see details in [70].
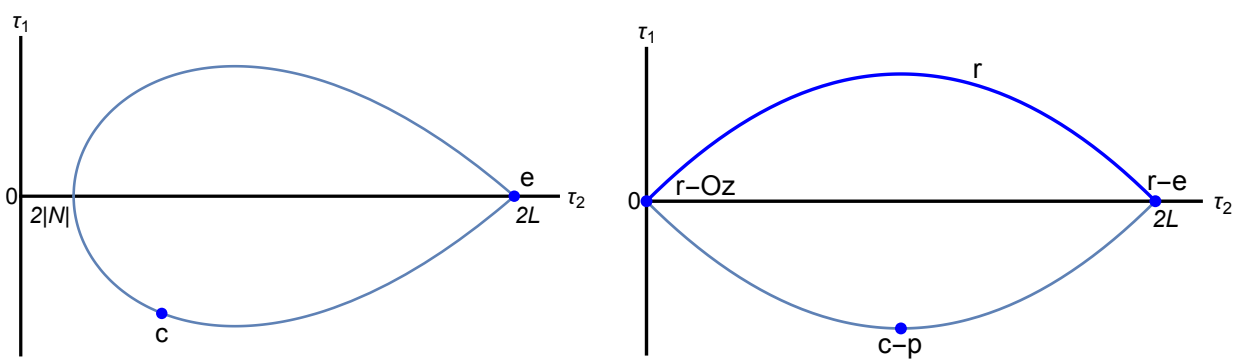

Figure 2. Sections $\tau_{3}=0$ of a balloon and a lemon, showing special types of trajectories. On the left, $c$ stands for the family of circular trajectories while $e$ denotes the family of equatorial motions. On the right, $r$ represents the arc corresponding to rectilinear trajectories, while $r$ - $O z$ are the trajectories in the $O z$-axis, $r$-e denotes rectilinear motions in the equatorial plane and $c-p$ are circular polar trajectories.

Remark 7. To perform the reconstruction from the twice reduced space to $T^{*} \mathbb{R}^{3}$ through $\mathbb{C P}_{h}^{2}$ (or through $\mathcal{M}_{\gamma}$ ), one has to take into account that the regular points of the balloon and lemon give rise to families of 2-tori. The same happens with the 
singularities $S_{1}$ and $S_{3}$. However, from the singularity $S_{2}$ one reconstructs families of periodic solutions, those in the OzZ-plane. The reason for this is that even when passing from $\mathcal{T}_{L, 0}$ to $\mathcal{M}_{0}$, to the singularity corresponding to the origin of the lemon one attaches an $S^{1}$, leading to a family of (rectilinear) motions, when reconstructing from $\mathcal{M}_{0}$ to $T^{*} \mathbb{R}^{3}$, this family gets transformed into the same family since this type of rectilinear motions is in the singular subspace of $\mathcal{M}_{0}$. See more details in $[16,31]$.

5.3. Twice reduced Hamiltonian and equations of motion. At this stage we need to express Hamiltonian (34) as a function of the invariants defined in (52). To achieve this we build a Gröbner basis from the polynomials relating the $\tau_{j}$ with the $\rho_{k}$ including the fundamental constraints of both sets of invariants. The Gröbner basis is computed with respect to the $\rho_{k}$. Then we apply the division algorithm for multivariate polynomials and divide (34) with respect to the Gröbner basis. The resulting remainder in this operation is the twice reduced Hamiltonian in terms of the invariants (50), which after simplifying the expressions and dropping constant terms can be written as

$$
\mathcal{K}=\alpha \tau_{1}+\beta \tau_{2}+\delta \tau_{2}^{2},
$$

where $\alpha, \beta$ and $\delta$ are parameters given by

$$
\begin{aligned}
& \alpha=\frac{1}{8}\left[a_{5}\left(a_{10}-2 a_{5}\right)+b_{10}\right], \\
& \beta=-\frac{1}{12}\left(4 a_{5}^{2}+18 a_{5} a_{10}-45 a_{10}^{2}-6 b_{10}+18 b_{15}\right) L, \\
& \delta=\frac{1}{16}\left(a_{5}^{2}+12 a_{5} a_{10}-15 a_{10}^{2}+6 b_{1}-4 b_{10}+6 b_{15}\right) .
\end{aligned}
$$

Alternatively, a rotation in the $\left(\tau_{1}, \tau_{3}\right)$-plane can be performed to remove the linear term in $\tau_{3}$ and obtain a polynomial like (56). This polynomial is, in fact, the most general expression attainable from a fourth-order Hamiltonian on $T^{*} \mathbb{R}^{3}$. 
The associated differential system is

$$
\begin{aligned}
& \dot{\tau}_{1}=-4\left(\beta+2 \delta \tau_{2}\right) \tau_{3} \\
& \dot{\tau}_{2}=4 \alpha \tau_{3} \\
& \dot{\tau}_{3}=4\left[\left(\beta+2 \delta \tau_{2}\right) \tau_{1}-2 \alpha\left(2 L-\tau_{2}\right)\left(\tau_{2}^{2}-L \tau_{2}-2 N^{2}\right)\right] .
\end{aligned}
$$

For the sake of simplifying the calculations and decreasing the number of parameters of the system we perform the following scaling

$$
\tau_{1}=L^{2} \tilde{\tau}_{1}, \quad \tau_{2}=L \tilde{\tau}_{2}, \quad \tau_{3}=L^{2} \tilde{\tau}_{3}, \quad \delta=\tilde{\delta} /(2 L), \quad \alpha=\tilde{\alpha} /(2 L), \quad \sigma=N / L
$$

Next, after rescaling time $(t \rightarrow(L / 2) t)$, Hamiltonian (56) is transformed into

$$
\mathcal{K}=\tilde{\alpha} \tilde{\tau}_{1}+2 \beta \tilde{\tau}_{2}+\tilde{\delta} \tilde{\tau}_{2}^{2}
$$

and system (58) becomes

$$
\begin{aligned}
& \tilde{\tau}_{1}^{\prime}=-\frac{8}{L}\left(\beta+\tilde{\delta} \tilde{\tau}_{2}\right) \tilde{\tau}_{3}, \\
& \tilde{\tau}_{2}^{\prime}=\frac{4}{L} \tilde{\alpha} \tilde{\tau}_{3}, \\
& \tilde{\tau}_{3}^{\prime}=\frac{8}{L}\left[\left(\beta+\tilde{\delta} \tilde{\tau}_{2}\right) \tilde{\tau}_{1}-\tilde{\alpha}\left(2-\tilde{\tau}_{2}\right)\left(\tilde{\tau}_{2}^{2}-\tilde{\tau}_{2}-2 \sigma^{2}\right)\right],
\end{aligned}
$$

where the primes denote derivation with respect to the rescaled time (a different time compared to the previous equations of motions given in (1), (12) and (35)). Constraint (51) results in

$$
\tilde{\tau}_{1}^{2}+\tilde{\tau}_{3}^{2}=\left(2-\tilde{\tau}_{2}\right)^{2}\left(\tilde{\tau}_{2}^{2}-4 \sigma^{2}\right)
$$

with $2|\sigma| \leq \tilde{\tau}_{2} \leq 2$ and the orbit space $\mathcal{T}_{\sigma}$ is given by

$$
\mathcal{T}_{\sigma}=\left\{\left(\tilde{\tau}_{1}, \tilde{\tau}_{2}, \tilde{\tau}_{3}\right) \in \mathbb{R}^{3}\left|\tilde{\tau}_{1}^{2}+\tilde{\tau}_{3}^{2}=\left(2-\tilde{\tau}_{2}\right)^{2}\left(\tilde{\tau}_{2}^{2}-4 \sigma^{2}\right), 2\right| \sigma \mid \leq \tilde{\tau}_{2} \leq 2\right\}
$$

Remark 8. When $\tilde{\alpha}=\beta=\tilde{\delta}=0$ then $\mathcal{K}=0$ and trivially the whole orbit space $\mathcal{T}_{\sigma}$ are relative equilibria. The system is not structurally stable. Nevertheless, these degeneracies disappear when the normal form computation (32) is pushed to order 
four in $\epsilon$.

Lemma 5.1. If $P^{*}=\left(\tilde{\tau}_{1}^{*}, \tilde{\tau}_{2}^{*}, \tilde{\tau}_{3}^{*}\right)$ is an equilibrium solution of system (60) with parameters $(\beta, \tilde{\delta}, \tilde{\alpha}, \sigma, L)$, then $P^{*}$ is also an equilibrium solution of (60) with parameters $(-\beta,-\tilde{\delta},-\tilde{\alpha}, \sigma, L)$. Moreover, if $\lambda$ is an eigenvalue corresponding to the linearisation of (60) around $P^{*}$ with parameters $(\beta, \tilde{\delta}, \tilde{\alpha}, \sigma, L)$, then $-\lambda$ is an eigenvalue corresponding to the linearisation of $P^{*}$ with parameters $(-\beta,-\tilde{\delta},-\tilde{\alpha}, \sigma, L)$.

Proof The first part of the lemma follows by simple inspection of the vector field (60). For the second part notice that the matrix associated to the linearised system around equilibrium $P^{*}$ is

$$
A=A\left((\beta, \tilde{\delta}, \tilde{\alpha}, \sigma, L),\left(\tilde{\tau}_{1}^{*}, \tilde{\tau}_{2}^{*}, \tilde{\tau}_{3}^{*}\right)\right)=\left[\begin{array}{ccc}
0 & v_{12} & v_{13} \\
0 & 0 & \frac{4}{L} \tilde{\alpha} \\
-v_{13} & v_{32} & 0
\end{array}\right],
$$

with

$v_{12}=-\frac{8}{L} \tilde{\delta} \tilde{\tau}_{3}^{*}, \quad v_{13}=-\frac{8}{L}\left(\beta+\tilde{\delta} \tilde{\tau}_{2}^{*}\right), \quad v_{32}=\frac{8}{L}\left\{\tilde{\delta} \tilde{\tau}_{1}^{*}+\tilde{\alpha}\left[2-2 \sigma^{2}+3\left(\tilde{\tau}_{2}^{*}-2\right) \tilde{\tau}_{2}^{*}\right]\right\}$.

Thence,

$$
A\left((-\beta,-\tilde{\delta},-\tilde{\alpha}, \sigma, L),\left(\tilde{\tau}_{1}^{*}, \tilde{\tau}_{2}^{*}, \tilde{\tau}_{3}^{*}\right)\right)=-A\left((\beta, \tilde{\delta}, \tilde{\alpha}, \sigma, L),\left(\tilde{\tau}_{1}^{*}, \tilde{\tau}_{2}^{*}, \tilde{\tau}_{3}^{*}\right)\right)
$$

and the second part of the lemma follows immediately.

Lemma 5.2. System (60) with parameters $(\beta, \tilde{\delta}, \tilde{\alpha}, \sigma, L)$ is transformed under the reflection $\left(\tilde{\tau}_{1}, \tilde{\tau}_{2}, \tilde{\tau}_{3}\right) \rightarrow\left(-\tilde{\tau}_{1}, \tilde{\tau}_{2}, \tilde{\tau}_{3}\right)$ into the same system with parameters $(-\beta,-\tilde{\delta}, \tilde{\alpha}, \sigma, L)$. In particular, if $P^{*}=\left(\tilde{\tau}_{1}^{*}, \tilde{\tau}_{2}^{*}, \tilde{\tau}_{3}^{*}\right)$ is an equilibrium of (60) with parameters $(\beta, \tilde{\delta}, \tilde{\alpha}, \sigma, L)$, then $\bar{P}^{*}=\left(-\tilde{\tau}_{1}^{*}, \tilde{\tau}_{2}^{*}, \tilde{\tau}_{3}^{*}\right)$ is also an equilibrium of (60) with parameters $(-\beta,-\tilde{\delta}, \tilde{\alpha}, \sigma, L)$. Moreover, both have the same stability character. 
Proof The first part of the lemma follows directly by inspection of vector field (60).

For the second part, according to Lemma 5.1 , if $A\left((\beta, \tilde{\delta}, \tilde{\alpha}, \sigma, L),\left(\tilde{\tau}_{1}^{*}, \tilde{\tau}_{2}^{*}, \tilde{\tau}_{3}^{*}\right)\right)$ is the matrix associated to the linearisation around the equilibrium $P^{*}=\left(\tilde{\tau}_{1}^{*}, \tilde{\tau}_{2}^{*}, \tilde{\tau}_{3}^{*}\right)$, then, the matrix associated to $\bar{P}^{*}=\left(-\tilde{\tau}_{1}^{*}, \tilde{\tau}_{2}^{*}, \tilde{\tau}_{3}^{*}\right)$ is given by

$$
A\left((-\beta,-\tilde{\delta}, \tilde{\alpha}, \sigma, L),\left(-\tilde{\tau}_{1}^{*}, \tilde{\tau}_{2}^{*}, \tilde{\tau}_{3}^{*}\right)\right)=\left[\begin{array}{ccc}
0 & -v_{12} & -v_{13} \\
0 & 0 & \frac{4}{L} \tilde{\alpha} \\
v_{13} & v_{32} & 0
\end{array}\right] .
$$

It can be checked that both matrices share the characteristic polynomial

$$
p(\lambda)=-\lambda^{3}+\left(\frac{4}{L} \tilde{\alpha} v_{32}-v_{13}^{2}\right) \lambda-\frac{4}{L} \tilde{\alpha} v_{12} v_{13} .
$$

5.4. The equilibria at the singular points. The singular point $\mathrm{P}_{1}$ with coordinates $\left(\tilde{\tau}_{1}, \tilde{\tau}_{2}, \tilde{\tau}_{3}\right)=(0,2,0)$ is at an equilibrium for all the values of the parameters. When $\sigma=0$ the point $\mathrm{P}_{2}$ with coordinates $\left(\tilde{\tau}_{1}, \tilde{\tau}_{2}, \tilde{\tau}_{3}\right)=(0,2|\sigma|, 0)$ is also singular and then is at an equilibrium. In this subsection we analyse the stability of both points. For that, we perform a blowup of the singularities in an analogous way as was done in [31].

Proposition 5.2. The stability of the equilibrium point $\mathrm{P}_{1}$ changes for $|\tilde{\alpha}| \sqrt{1-\sigma^{2}}$ $=|\beta+2 \tilde{\delta}|$, what corresponds to a bifurcation. More precisely, when $|\tilde{\alpha}| \sqrt{1-\sigma^{2}}<$ $|\beta+2 \tilde{\delta}|$ then $\mathrm{P}_{1}$ is stable, when $|\tilde{\alpha}| \sqrt{1-\sigma^{2}}>|\beta+2 \tilde{\delta}|$ it is unstable whereas for $|\tilde{\alpha}| \sqrt{1-\sigma^{2}}=|\beta+2 \tilde{\delta}|$ it is degenerate.

Proof To study the local behaviour of $\mathrm{P}_{1}$ we pass to the $2: 1$ covering given by

$$
\tilde{\tau}_{1}=\frac{1}{4}\left(u^{2}-v^{2}\right), \quad \tilde{\tau}_{2}=1+w, \quad \tilde{\tau}_{3}=\frac{1}{2} u v .
$$

The constraint (61) defining the twice reduced space is transformed into

$$
\frac{1}{16}\left(u^{2}+v^{2}\right)^{2}-(1-w)^{2}\left[(1+w)^{2}-4 \sigma^{2}\right]=0
$$


Notice that $u, v$ are not symplectic coordinates. Since we are interested in the analysis around $\left(\tilde{\tau}_{1}, \tilde{\tau}_{2}, \tilde{\tau}_{3}\right)=(0,2,0)$, then $w \lesssim 1$ and in order to obtain the correct time scale we replace (65) by the equivalent relation

$$
Q_{\sigma}(u, v, w)=\frac{1}{4}\left(u^{2}+v^{2}\right)-(1-w) \sqrt{(1+w)^{2}-4 \sigma^{2}}=0 .
$$

Hamiltonian (59) turns into

$$
\mathcal{K}(u, v, w)=\frac{\tilde{\alpha}}{4}\left(u^{2}-v^{2}\right)+2 \beta(1+w)+\tilde{\delta}(1+w)^{2} .
$$

Next, we use (66) to express $w=w_{\sigma}(u, v)$. We replace $w$ in (67) and develop in Taylor series around $(u, v)=(0,0)$ to get

$$
\begin{aligned}
\mathcal{K}(u, v)= & 4(\beta+\tilde{\delta})+\frac{\tilde{\alpha} \sqrt{1-\sigma^{2}}-\beta-2 \tilde{\delta}}{4 \sqrt{1-\sigma^{2}}} u^{2}-\frac{\tilde{\alpha} \sqrt{1-\sigma^{2}}+\beta+2 \tilde{\delta}}{4 \sqrt{1-\sigma^{2}}} v^{2} \\
& -\frac{\beta+\tilde{\delta}\left(1+\sigma^{2}\right)}{64\left(1-\sigma^{2}\right)^{2}}\left(u^{2}+v^{2}\right)^{2}-\frac{4(\beta+\tilde{\delta})+(\beta+6 \tilde{\delta}) \sigma^{2}}{2048\left(1-\sigma^{2}\right)^{7 / 2}}\left(u^{2}+v^{2}\right)^{3}+\cdots,
\end{aligned}
$$

where $\ldots$ means higher order terms in the variables $u, v$. Note that by construction the system is equivariant with respect to the $\pi$-rotation $(u, v, w) \rightarrow(-u,-v, w)$, i.e., the system on the $2: 1$ covering is $\mathbb{Z}_{2}$-symmetric.

Now, we arrive at the following cases:

(1) When $|\tilde{\alpha}| \sqrt{1-\sigma^{2}}<|\beta+2 \tilde{\delta}|$ then, the sign of the coefficients of $u^{2}$ and $v^{2}$ is the same. Thus, $(0,0)$ is a centre and therefore, $\mathrm{P}_{1}$ is a centre.

(2) When $|\tilde{\alpha}| \sqrt{1-\sigma^{2}}>|\beta+2 \tilde{\delta}|$ then, the sign of the coefficients of $u^{2}$ and $v^{2}$ are opposite. Thus, $(0,0)$ is a saddle. Due to the $2: 1$ covering $\mathrm{P}_{1}$ will appear as "half a saddle", i.e. a cone-like point.

(3) When $|\tilde{\alpha}| \sqrt{1-\sigma^{2}}=|\beta+2 \tilde{\delta}|$ then, either the coefficient of $u^{2}$ or the coefficient of $v^{2}$ or both of them are 0 . Thus, $(0,0)$ is degenerate, and we have to analyse the fourth-order terms in $(u, v)$. For this relation of the parameters a bifurcation takes place as $(0,0)$ changes from stable to unstable. 
In Theorem 5.1 we will prove that when $\tilde{\alpha}=0$ this bifurcation is degenerate and $\mathrm{P}_{1}$ is stable. In Theorem 5.2 we will see that the cases $\tilde{\alpha} \neq 0$ and $(\beta, \tilde{\delta}, \sigma) \in\{(-1 / \tilde{\alpha}, 1 / \tilde{\alpha}, 0),(1 / \tilde{\alpha},-1 / \tilde{\alpha}, 0)\}$ also correspond to degenerate bifurcations. In the rest of the cases $\mathrm{P}_{1}$ undergoes a Hamiltonian flip bifurcation, as it will be shown in Theorem 5.4.

Proposition 5.3. The stability of the point $\mathrm{P}_{2}=(0,0,0)$ on $\mathcal{T}_{0}$ changes when $|\tilde{\alpha}|=|\beta|$. More precisely, when $|\tilde{\alpha}|<|\beta|$ then $\mathrm{P}_{2}$ is stable, and when $|\tilde{\alpha}|>|\beta|$, it is unstable. For $|\tilde{\alpha}|=|\beta|$ the point $\mathrm{P}_{2}$ undergoes a bifurcation.

Proof For the proof of this lemma we use the same change of variables as the one given in the proof of Proposition 5.2. As in this case we are concerned with the analysis around $\left(\tilde{\tau}_{1}, \tilde{\tau}_{2}, \tilde{\tau}_{3}\right)=(0,0,0)$, then $w \gtrsim-1$ and

$$
Q_{0}(u, v, w)=\frac{1}{4}\left(u^{2}+v^{2}\right)-\left(1-w^{2}\right)=0 .
$$

Now, we use (69) to express $w$ as a function of $(u, v)$, we substitute it into the expression of Hamiltonian (68) and compute the Taylor expansion around $(0,0)$ to get

$$
\mathcal{K}(u, v)=\frac{1}{4}(\tilde{\alpha}+\beta) u^{2}-\frac{1}{4}(\tilde{\alpha}-\beta) v^{2}+\frac{1}{64}(\beta+\tilde{\delta})\left(u^{2}+v^{2}\right)^{2}+\cdots .
$$

If $|\tilde{\alpha}|<|\beta|$ the sign of the coefficients of $u^{2}$ and $v^{2}$ is the same, then $(0,0)$ is a centre and $\mathrm{P}_{2}$ is also a centre. When $|\tilde{\alpha}|>|\beta|$ we get a saddle point on the $(u, v)$-space. Since these coordinates represent a 2:1 covering, it follows that $\mathrm{P}_{2}$ is a cone-like point. When $|\tilde{\alpha}|=|\beta|$, either the coefficient of $u^{2}$ or the coefficient of $v^{2}$ or both vanish and $(0,0)$ is degenerate. Thus, we have to analyse the next term in the Taylor series. For this relation of the parameters a bifurcation involving $\mathrm{P}_{2}$ takes place, as its stability character changes. In Theorem 5.1 we will prove that when $\tilde{\alpha}=\beta=0$ this bifurcation is degenerate although $\mathrm{P}_{2}$ is stable. In Theorem 5.2 we will prove that when $\tilde{\alpha} \neq 0$ and $(\beta, \tilde{\delta}, \sigma) \in\{(-1 / \tilde{\alpha}, 1 / \tilde{\alpha}, 0),(1 / \tilde{\alpha},-1 / \tilde{\alpha}, 0)\}$ we get 
degenerate bifurcations. In the rest of the cases $\mathrm{P}_{2}$ undergoes Hamiltonian Hopf bifurcations, as it will be shown in Theorem 5.5. The degenerate cases will be discussed in Subsection 5.6.

Note 5.1. The stability of $\mathrm{P}_{2}$ analysed in (5.3) agrees with the stability of the point $\mathcal{O}_{R}$ studied in Theorem 4.2 as both points represent the same type of solutions, which is apparent by using the relationships between the parameters given in (57). Notice in particular that condition $\omega_{1} \omega_{2}>0$ in Theorem 4.2 is equivalent to condition $|\tilde{\alpha}|<|\beta|$. Indeed the reduction process from $\mathbb{C P}_{h}^{2}$ to $\mathcal{T}_{L, N}$ carries the point $\mathcal{O}_{R}$ into $\mathrm{P}_{2}$.

5.5. The equilibria at the regular points. In this subsection we analyse the stationary solutions of system (60) together with the constraint (61). As the twice reduced space is different for $\sigma=0$ and $\sigma \neq 0$ we will distinguish between the two cases. Recall that when $\sigma=0$ the two singularities, $\mathrm{P}_{1}$ and $\mathrm{P}_{2}$, are equilibrium points and for $\sigma \neq 0$ the unique singularity $\mathrm{P}_{1}$ is also an equilibrium.

From the second equation of system (60) there are two possibilities, either $\tilde{\alpha}=0$ or $\tilde{\tau}_{3}=0$.

5.5.1. Case $\tilde{\alpha}=0$. System (60) reads

$$
\begin{aligned}
& \tilde{\tau}_{1}^{\prime}=-\frac{8}{L}\left(\beta+\tilde{\delta} \tilde{\tau}_{2}\right) \tilde{\tau}_{3}, \\
& \tilde{\tau}_{2}^{\prime}=0, \\
& \tilde{\tau}_{3}^{\prime}=\frac{8}{L}\left(\beta+\tilde{\delta} \tilde{\tau}_{2}\right) \tilde{\tau}_{1},
\end{aligned}
$$

For convenience we define the following parametric sets:

$$
\begin{aligned}
& \mathcal{Y}_{\sigma}=\left\{(\beta, \tilde{\delta}, \sigma) \in \mathbb{R}^{3} \mid \beta=\tilde{\delta}=0,-1 \leq \sigma \leq 1\right\}, \\
& \mathcal{A}_{\sigma}=\left\{(\beta, \tilde{\delta}, \sigma) \in \mathbb{R}^{3}|2| \sigma \mid<-\beta / \tilde{\delta}<2,-1 \leq \sigma \leq 1, \tilde{\delta} \neq 0\right\}, \\
& \mathcal{L}_{\sigma}=\left\{(\beta, \tilde{\delta}, \sigma) \in \mathbb{R}^{3}|-\beta / \tilde{\delta}=2| \sigma \mid,-1 \leq \sigma \leq 1, \tilde{\delta} \neq 0\right\}, \\
& \mathcal{N}_{\sigma}=\left\{(\beta, \tilde{\delta}, \sigma) \in \mathbb{R}^{3} \mid-\beta / \tilde{\delta}=2,-1 \leq \sigma \leq 1, \tilde{\delta} \neq 0\right\} .
\end{aligned}
$$


We denote $\mathcal{Y}_{0}=\mathcal{Y}_{\sigma} \cap\{\sigma=0\}, \mathcal{A}_{0}=\mathcal{A}_{\sigma} \cap\{\sigma=0\}, \mathcal{L}_{0}=\mathcal{L}_{\sigma} \cap\{\sigma=0\}$ and $\mathcal{N}_{0}=\mathcal{N}_{\sigma} \cap\{\sigma=0\}$. We also indicate with $\mathcal{Y}, \mathcal{A}, \mathcal{L}, \mathcal{N}$ the projections of $\mathcal{Y}_{\sigma}, \mathcal{A}_{\sigma}$, $\mathcal{L}_{\sigma}, \mathcal{N}_{\sigma}$ onto their first two components, respectively.

Theorem 5.1. When $\tilde{\alpha}=0$ the two-parameter family defined by $\mathcal{K}=2 \beta \tilde{\tau}_{2}+\tilde{\delta} \tilde{\tau}_{2}^{2}$ on $\mathcal{T}_{\sigma}$ is structurally stable in the parametric region $\mathbb{R}^{3} \backslash(\mathcal{Y} \cup \mathcal{A} \cup \mathcal{L} \cup \mathcal{N})$. In $\mathbb{R}^{3} \backslash\left(\mathcal{Y}_{\sigma} \cup \mathcal{A}_{\sigma} \cup \mathcal{L}_{\sigma} \cup \mathcal{N}_{\sigma}\right)$ there are two stable equilibria given by $\mathrm{P}_{1}=(0,2,0)$ and $\mathrm{P}_{2}=(0,2|\sigma|, 0)$. In $\mathcal{Y}_{\sigma}$ all the points in $\mathcal{T}_{\sigma}$ are equilibria. In $\mathcal{A}_{\sigma}$, apart from the stable equilibria $\mathrm{P}_{1}=(0,2,0)$ and $\mathrm{P}_{2}=(0,2|\sigma|, 0)$, there is a curve of equilibria given by $\left(\tilde{\tau}_{1},-\beta / \tilde{\delta}, \tilde{\tau}_{3}\right)$ where $\tilde{\tau}_{1}^{2}+\tilde{\tau}_{3}^{2}=(2+\beta / \tilde{\delta})^{2}\left(\beta^{2} / \tilde{\delta}^{2}-4 \sigma^{2}\right)$. In $\mathcal{L}_{\sigma}$ the equilibrium $\mathrm{P}_{2}$ undergoes a degenerate bifurcation and in $\mathcal{N}_{\sigma}$ it is the equilibrium $\mathrm{P}_{1}$ that undergoes a degenerate bifurcation.

Proof From system (71) one clearly has either $\beta+\tilde{\delta} \tilde{\tau}_{2}=0$ or $\tilde{\tau}_{1}=\tilde{\tau}_{3}=0$.

(1) When $\tilde{\tau}_{1}=\tilde{\tau}_{3}=0$, taking into account (61) it is immediate to conclude that for any value of the parameters we end up with the point $\mathrm{P}_{1}=(0,2,0)$ and $\mathrm{P}_{2}=(0,2|\sigma|, 0)$. The points $\mathrm{P}_{1}$ and $\mathrm{P}_{2}=(0,0,0)$ correspond to the singularities considered in the previous subsection.

(2) For $\beta+\tilde{\delta} \tilde{\tau}_{2}=0$ we distinguish between two possibilities:

- When $\tilde{\delta}=0$ then $\beta=0$ and, since $\tilde{\alpha}$ is also zero, we are in the case of Remark 8 where all the points in the twice reduced space are equilibria and the system is not structurally stable. This is the parametric set $\mathcal{Y}_{\sigma}$, that corresponds to the vertical line through the origin in Fig. 3. The orbit space for $\mathcal{Y}_{0}$ is represented in Fig. 4.

- If $\tilde{\delta} \neq 0$ then $\tilde{\tau}_{2}=-\beta / \tilde{\delta}$ and, taking into account (61), we end up with the following description: 
(i) When $2|\sigma|<-\beta / \tilde{\delta}<2$, i.e. in $\mathcal{A}_{\sigma}$, the points $\left(\tilde{\tau}_{1},-\beta / \tilde{\delta}, \tilde{\tau}_{3}\right)$ with $\tilde{\tau}_{1}^{2}+\tilde{\tau}_{3}^{3}=(2+\beta / \tilde{\delta})^{2}\left(\beta^{2} / \tilde{\delta}^{2}-4 \sigma^{2}\right)$ are equilibria. The set $\mathcal{A}_{\sigma}$ is the region enclosed by the red and blue surfaces in Fig. 3. It corresponds to the yellow region in Fig. 4.

(ii) When $2|\sigma|=-\beta / \tilde{\delta}$, i.e. in region $\mathcal{L}_{\sigma}$, then this set of equilibria gets reduced to the point $\mathrm{P}_{2}=(0,2|\sigma|, 0)$, that we know is already an equilibrium. Thus, the parametric relation $\beta+2 \tilde{\delta}|\sigma|=0$ corresponds to a degenerate bifurcation related to $\mathrm{P}_{2}$, see the blue surface in Fig. 3. Notice that, when $\sigma=0$, this is the bifurcation mentioned in Proposition 5.3 for $\tilde{\alpha}=0$ and is represented in blue in Fig. 4 .

(iii) When $-\beta / \tilde{\delta}=2$, i.e. in region $\mathcal{N}_{\sigma}$, then the set of equilibria gets reduced to $\mathrm{P}_{1}$, that we already know is an equilibrium. Thus, the parametric relation $\beta+2 \tilde{\delta}=0$ corresponds to a degenerate bifurcation related to $\mathrm{P}_{1}$, see the red surface in Fig. 3. Note that it is the relation appearing in Proposition 5.2 when $\tilde{\alpha}=0$ and is represented in red in Fig. 4 .

In summary, when $2|\sigma|<-\beta / \tilde{\delta}<2$ there are two isolated equilibria, $\mathrm{P}_{1}$ and $\mathrm{P}_{2}$, and a curve of equilibria given by $\left(\tilde{\tau}_{1},-\beta / \tilde{\delta}, \tilde{\tau}_{3}\right)$ with $\tilde{\tau}_{1}^{2}+\tilde{\tau}_{3}^{3}=(2+\beta / \tilde{\delta})^{2}\left(\beta^{2} / \tilde{\delta}^{2}-\right.$ $\left.4 \sigma^{2}\right)$, see a sketch of the phase space for $\sigma=0$ together with the equilibria in the yellow region appearing in Fig. 4 . When $-\beta / \tilde{\delta}=2|\sigma|$ the curve of equilibria collides with $\mathrm{P}_{2}$, and only $\mathrm{P}_{1}$ and $\mathrm{P}_{2}$ remain as equilibria for $-\beta / \tilde{\delta} \leq 2|\sigma|$, see the reduced space for $\sigma=0$ corresponding to the blue line and the white region next to it in Fig. 4. Finally, when $-\beta / \tilde{\delta}=2$ the curve of equilibria collides with $\mathrm{P}_{1}$, and only $\mathrm{P}_{1}$ and $\mathrm{P}_{2}$ remain as equilibria for $-\beta / \tilde{\delta} \geq 2$, see the reduced space for $\sigma=0$ corresponding to the red line and the white region next to it in Fig. 4.

Let us prove that the isolated equilibria are always stable points, as we can see in Fig. 4.

- As the Poincaré index of $\mathcal{T}_{\sigma}$ is 2 , in the case where there are only two equilibria, both have index 1 , so they are stable (centres). This occurs when 
$\tilde{\delta} \neq 0$ and $-\beta / \tilde{\delta} \geq 2$ and also when $\tilde{\delta} \neq 0$ and $-\beta / \tilde{\delta} \leq 2|\sigma|$.

- In region $\mathcal{A}_{\sigma}$, applying Proposition 5.2 we know that $\mathrm{P}_{1}$ is stable when $|\tilde{\alpha}| \sqrt{1-\sigma^{2}}<|\beta+2 \tilde{\delta}|$. Since $\tilde{\alpha}=0$, we trivially conclude that $\mathrm{P}_{1}$ is stable. For $\mathrm{P}_{2}$ we get the following:

(i) When $\sigma=0$ by Proposition 5.3 , we know that $\mathrm{P}_{2}=(0,0,0)$ is stable when $|\tilde{\alpha}|<|\beta|$. As $\tilde{\alpha}=0$, it is immediate to conclude that $\mathrm{P}_{2}$ is stable.

(ii) When $\sigma \neq 0, \mathrm{P}_{2}$ is a regular point. Thus, its stability is given by the matrix (63) associated to the linearisation of system (60) around $\mathrm{P}_{2}$. The non-trivial eigenvalues are $\pm 8 i(\beta+2 \tilde{\delta}|\sigma|) / L$. So, in region $\mathcal{A}_{\sigma}$ with $\sigma \neq 0$, $\mathrm{P}_{2}$ is stable.

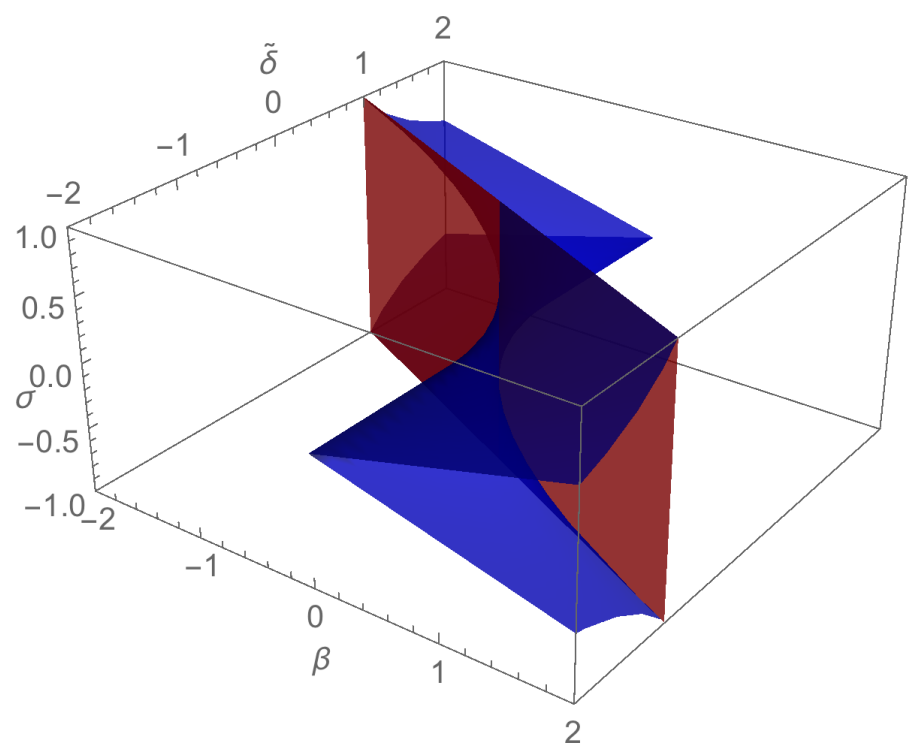

Figure 3. Bifurcation diagram for $\tilde{\alpha}=0$. The vertical line through the origin corresponds to $\mathcal{Y}_{\sigma}$; the blue surface is $\mathcal{L}_{\sigma}$ (a degenerate bifurcation of $\mathrm{P}_{2}$ ) and the red surface is $\mathcal{N}_{\sigma}$ (a degenerate bifurcation of $\mathrm{P}_{1}$ ); the region enclosed by the red and blue surfaces stands for the set $\mathcal{A}_{\sigma}$.

The analysis of the case $\tilde{\alpha}=0$ is collected in Figs. 3 and 4 . Fig. 4 is the intersection with the plane $\sigma=0$ of the bifurcation diagram contained in Fig. 3. 


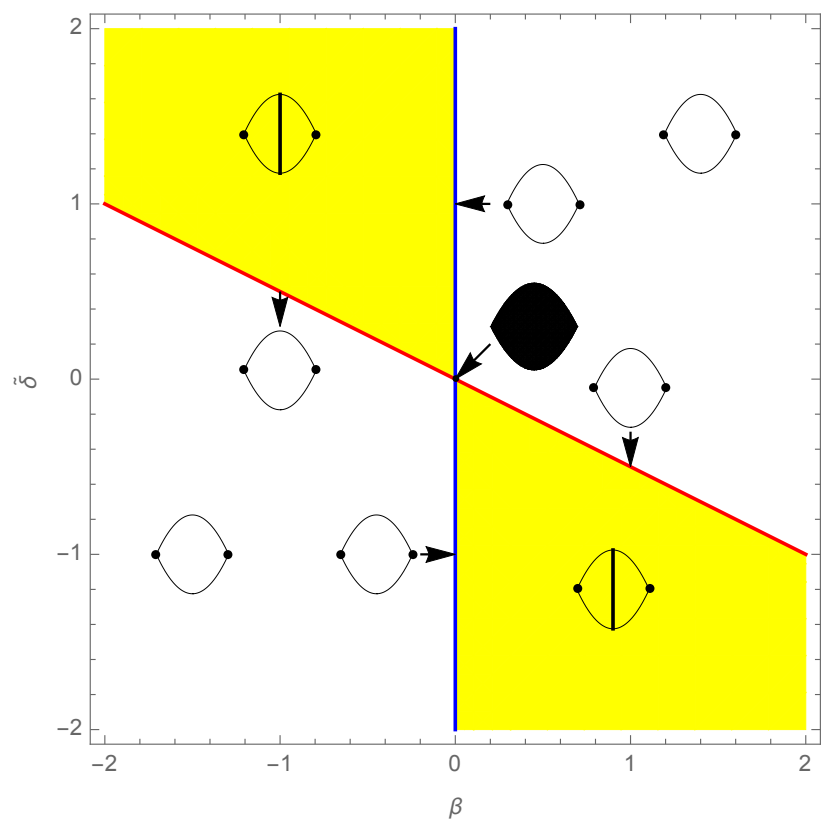

Figure 4. Intersection with the plane $\sigma=0$ of the bifurcation diagram in the case $\tilde{\alpha}=0$. The origin corresponds to $\mathcal{Y}_{0}$; the yellow region stands for the set $\mathcal{A}_{0}$; the blue line is $\mathcal{L}_{0}$ (a degenerate bifurcation of $\mathrm{P}_{2}$ ) and the red line is $\mathcal{N}_{0}$ (a degenerate bifurcation of $\mathrm{P}_{1}$ ). A sketch of the twice reduced space projected over $\tilde{\tau}_{3}=0$ together with the equilibria is represented for each parametric set. Black dots correspond to stable equilibria (Poincaré index 1). At the origin the whole phase space are equilibria. In the yellow region there is a curve of equilibria with constant $\tilde{\tau}_{2}$.

The blue (red) line in Fig. 4 is the intersection with $\sigma=0$ of the blue (red) surface in Fig. 3. The yellow region in Fig. 4 corresponds to the surface enclosed by the red and blue surfaces in Fig. 3. A sketch of the twice reduced phase space together with the equilibria and stability is given for each region in Fig. 4 . In the case $\sigma \neq 0$ the same sketches apply for the corresponding regions except for the shape of the twice reduced space, if $\sigma=0$ the point $\mathrm{P}_{2}=(0,0,0)$ is singular whereas when $\sigma \neq 0$ the equilibrium $\mathrm{P}_{2}=(0,2|\sigma|, 0)$ is regular.

5.5.2. Case $\tilde{\alpha} \neq 0$. As it has been noticed before, from system (60) we infer that in this case all the equilibria satisfy $\tilde{\tau}_{3}=0$. Thus, constraint (61) gets transformed 
into

$$
\tilde{\tau}_{1}^{2}=\left(2-\tilde{\tau}_{2}\right)^{2}\left(\tilde{\tau}_{2}^{2}-4 \sigma^{2}\right)
$$

We can reduce the number of parameters of the system by one defining

$$
\beta=\hat{\beta} \tilde{\alpha}, \quad \tilde{\delta}=\hat{\delta} \tilde{\alpha} .
$$

Rescaling time $(t \rightarrow \tilde{\alpha} t)$ Hamiltonian (59) is transformed into Hamiltonian

$$
\mathcal{K}=\tilde{\tau}_{1}+2 \hat{\beta} \tilde{\tau}_{2}+\hat{\delta} \tilde{\tau}_{2}^{2}
$$

and taking also into account that $\tilde{\tau}_{3}=0$ system (60) is transformed into

$$
\begin{aligned}
& \dot{\tilde{\tau}}_{1}=0, \\
& \dot{\tilde{\tau}}_{2}=0, \\
& \dot{\tilde{\tau}}_{3}=\frac{8}{L}\left[\left(\hat{\beta}+\hat{\delta} \tilde{\tau}_{2}\right) \tilde{\tau}_{1}+\left(2-\tilde{\tau}_{2}\right)\left(2 \sigma^{2}+\tilde{\tau}_{2}-\tilde{\tau}_{2}^{2}\right)\right],
\end{aligned}
$$

where (by an abuse of notation) the dots indicate derivation with respect to the new time. Then, the relative equilibria satisfy $\dot{\tilde{\tau}}_{3}=0$ and (72). Thus, we compute the resultant of both polynomials with respect to $\tau_{1}$ and get the sixth-degree polynomial in $\tau_{2}$ given by

$$
\mathcal{P}_{6}\left(\tilde{\tau}_{2}\right)=\frac{64}{L^{2}}\left(\tilde{\tau}_{2}-2\right)^{2} \mathcal{P}_{4}\left(\tilde{\tau}_{2}\right)
$$

where

$$
\begin{aligned}
\mathcal{P}_{4}\left(\tilde{\tau}_{2}\right)= & 4 \sigma^{2}\left(\hat{\beta}^{2}+\sigma^{2}\right)+4 \sigma^{2}(1+2 \hat{\beta} \hat{\delta}) \tilde{\tau}_{2}+\left[1-\hat{\beta}^{2}-4 \sigma^{2}\left(1-\hat{\delta}^{2}\right)\right] \tilde{\tau}_{2}^{2} \\
& -2(1+\hat{\beta} \hat{\delta}) \tilde{\tau}_{2}^{3}+\left(1-\hat{\delta}^{2}\right) \tilde{\tau}_{2}^{4} .
\end{aligned}
$$

The relative equilibria are the admissible solutions of $\mathcal{P}_{6}\left(\tilde{\tau}_{2}\right)=0$. The case $\tilde{\tau}_{2}=2$ is discarded as it corresponds to $\mathrm{P}_{1}$ and has been considered in Subsection 5.4. As we are treating regular points, we have to analyse the roots of $\mathcal{P}_{4}\left(\tilde{\tau}_{2}\right)$ such that $0<\tilde{\tau}_{2}<2$ and $\sigma=0$ or $2|\sigma| \leq \tilde{\tau}_{2}<2$ and $0<|\sigma|<1$. By convenience we define 
the sets:

$$
\begin{aligned}
& \mathcal{L}_{\sigma}=\left\{(\hat{\beta}, \hat{\delta}, \sigma) \in \mathbb{R}^{3} \mid \mathcal{Q}_{6}(\sigma)=0\right\}, \\
& \mathcal{L}_{\sigma}^{\prime}=\left\{(\hat{\beta}, \hat{\delta}, \sigma) \in \mathbb{R}^{3} \mid \mathcal{Q}_{6}(\sigma)=0, \hat{\delta}(\hat{\beta}+2 \hat{\delta})>1\right\}, \\
& \mathcal{L}_{0}^{1}=\left\{(\hat{\beta}, \hat{\delta}) \in \mathbb{R}^{2} \mid \hat{\beta}=-1, \hat{\delta} \neq 1\right\}, \\
& \mathcal{L}_{0}^{2}=\left\{(\hat{\beta}, \hat{\delta}) \in \mathbb{R}^{2} \mid \hat{\beta}=1, \hat{\delta} \neq-1\right\}, \mathcal{N}_{\sigma}^{1}=\left\{(\hat{\beta}, \hat{\delta}, \sigma) \in \mathbb{R}^{3} \backslash\{(1,-1,0)\} \mid \hat{\beta}+2 \hat{\delta}=-\sqrt{1-\sigma^{2}},-1 \leq \sigma \leq 1\right\}, \\
& \mathcal{N}_{\sigma}^{2}=\left\{(\hat{\beta}, \hat{\delta}, \sigma) \in \mathbb{R}^{3} \backslash\{(-1,1,0)\} \mid \hat{\beta}+2 \hat{\delta}=\sqrt{1-\sigma^{2}},-1 \leq \sigma \leq 1\right\}, \\
& \mathcal{C}_{0}^{1}=\left\{(\hat{\beta}, \hat{\delta}) \in \mathbb{R}^{2} \mid \hat{\beta}+2 \hat{\delta}<-1, \hat{\beta}<-1\right\}, \\
& \mathcal{C}_{0}^{2}=\left\{(\hat{\beta}, \hat{\delta}) \in \mathbb{R}^{2} \mid \hat{\beta}+2 \hat{\delta}>1, \hat{\beta}>1\right\}, \\
& \mathcal{D}_{0}=\left\{(\hat{\beta}, \hat{\delta}) \in \mathbb{R}^{2} \mid 0<\frac{1+\hat{\beta}}{1-\hat{\delta}}<2,0<\frac{1-\hat{\beta}}{1+\hat{\delta}}<2\right\}, \\
& \mathcal{E}_{0}^{1}=\left\{(\hat{\beta}, \hat{\delta}) \in \mathbb{R}^{2}|| \hat{\beta}+2 \hat{\delta}|<1,| \hat{\beta} \mid>1\right\}, \\
& \mathcal{E}_{0}^{2}=\left\{(\hat{\beta}, \hat{\delta}) \in \mathbb{R}^{2}|| \hat{\beta}+2 \hat{\delta}|>1,| \hat{\beta} \mid<1\right\},
\end{aligned}
$$

where $\mathcal{Q}_{6}(\sigma)$ is obtained as the relevant factor of the resultant between $\mathcal{P}_{4}\left(\tilde{\tau}_{2}\right)$ and $\mathcal{P}_{4}^{\prime}\left(\tilde{\tau}_{2}\right)$ with respect to $\tilde{\tau}_{2}$ and is explicitly given by

$$
\begin{aligned}
\mathcal{Q}_{6}(\sigma)= & \left(1-\hat{\beta}^{2}\right)^{3}+3\left[\left(5+\hat{\beta}^{2}\right)\left(1+5 \hat{\beta}^{2}\right)+36 \hat{\beta}\left(1+\hat{\beta}^{2}\right) \hat{\delta}+4\left(1+7 \hat{\beta}^{2}+\hat{\beta}^{4}\right) \hat{\delta}^{2}\right] \sigma^{2} \\
& +48\left(1-\hat{\beta}^{2}\right)\left(\hat{\delta}^{2}-1\right)^{2} \sigma^{4}+64\left(\hat{\delta}^{2}-1\right)^{3} \sigma^{6} .
\end{aligned}
$$

For $j=1,2$ we denote by $\mathcal{N}_{0}^{j}=\mathcal{N}_{\sigma}^{j} \cap\{\sigma=0\}$ and $\mathcal{N}^{j}$ the projection onto the first two components. We also indicate with $\mathcal{L}^{\prime}$ the projection of $\mathcal{L}_{\sigma}^{\prime}$ onto the plane defined by $\hat{\beta}, \hat{\delta}$. We establish the following result.

Theorem 5.2. When $\tilde{\alpha} \neq 0$ and $\sigma=0$ the two-parameter family defined by (74) on $\mathcal{T}_{0}$ is structurally stable in the parametric region $\mathbb{R}^{2} \backslash\left\{\left(\cup_{j=1}^{2} \mathcal{L}_{0}^{j}\right) \cup\left(\cup_{j=1}^{2} \mathcal{N}_{0}^{j}\right) \cup\right.$ $(-1,1) \cup(1,-1)\}=\left(\cup_{j=1}^{2} \mathcal{C}_{0}^{j}\right) \cup \mathcal{D}_{0} \cup\left(\cup_{j=1}^{2} \mathcal{E}_{0}^{j}\right)$. The equilibria and their stability character is as follows. 
- In the regions $\cup_{j=1}^{2} \mathcal{C}_{0}^{j} ; \mathcal{L}_{0}^{1}$ with $\hat{\delta} \leq 0 ; \mathcal{L}_{0}^{2}$ with $\hat{\delta} \geq 0 ; \mathcal{N}_{0}^{1}$ with $\hat{\beta} \leq-1$ and $\mathcal{N}_{0}^{2}$ with $\hat{\beta} \geq 1$ there are two stable equilibria, $\mathrm{P}_{1}$ and $\mathrm{P}_{2}=(0,0,0)$.

- In $\mathcal{D}_{0}$ there are four equilibria, i.e. $\mathrm{P}_{1}, \mathrm{P}_{2}$,

$$
\mathrm{P}_{3}=\left(\frac{(1+\hat{\beta})(1-\hat{\beta}-2 \hat{\delta})}{(1-\hat{\delta})^{2}}, \frac{1+\hat{\beta}}{1-\hat{\delta}}, 0\right), \mathrm{P}_{4}=\left(-\frac{(1-\hat{\beta})(1+\hat{\beta}+2 \hat{\delta})}{(1+\hat{\delta})^{2}}, \frac{1-\hat{\beta}}{1+\hat{\delta}}, 0\right) .
$$

(i) When $|\hat{\delta}|>1$ the points $\mathrm{P}_{1}, \mathrm{P}_{2}, \mathrm{P}_{3}$ are stable and $\mathrm{P}_{4}$ is unstable (a saddle).

(ii) When $|\hat{\delta}|<1$ the points $\mathrm{P}_{1}$ and $\mathrm{P}_{2}$ have index 0 and $\mathrm{P}_{3}$ and $\mathrm{P}_{4}$ are stable (centres).

- In the following situations there are three equilibria, specifically:

(i) In $\mathcal{E}_{0}^{1} \cup \mathcal{N}_{0}^{2}$ with $\hat{\beta}<-1$ and in $\mathcal{L}_{0}^{1}$ with $0<\hat{\delta}<1$ the points $\mathrm{P}_{2}$ and $\mathrm{P}_{4}$ are stable and $\mathrm{P}_{1}$ has index 0.

(ii) In $\mathcal{E}_{0}^{1} \cup \mathcal{N}_{0}^{1}$ with $\hat{\beta}>1$ and in $\mathcal{L}_{0}^{2}$ with $-1<\hat{\delta}<0$ the points $\mathrm{P}_{2}$ and $\mathrm{P}_{3}$ are stable and $\mathrm{P}_{1}$ has index 0 .

(iii) In $\mathcal{E}_{0}^{2}$ with $\hat{\beta}+2 \hat{\delta}<-1$; in $\mathcal{N}_{0}^{1}$ with $|\hat{\beta}|<1$; in $\mathcal{L}_{0}^{2}$ with $\hat{\delta}<-1$ the points $\mathrm{P}_{1}$ and $\mathrm{P}_{3}$ are stable and $\mathrm{P}_{2}$ has index 0 .

(iv) In $\mathcal{E}_{0}^{2}$ with $\hat{\beta}+2 \hat{\delta}>1$; in $\mathcal{N}_{0}^{2}$ with $|\hat{\beta}|<1$; in $\mathcal{L}_{0}^{1}$ with $\hat{\delta}>1$ the points $\mathrm{P}_{1}$ and $\mathrm{P}_{4}$ are stable and $\mathrm{P}_{2}$ has index 0 .

- The set $\left(\cup_{j=1}^{2} \mathcal{L}_{0}^{j}\right) \cup\left(\cup_{j=1}^{2} \mathcal{N}_{0}^{j}\right) \cup(-1,1) \cup(1,-1)$ is a bifurcation set.

(i) $\cup_{j=1}^{2} \mathcal{L}_{0}^{j}$ is a bifurcation associated to $\mathrm{P}_{2}$.

(ii) $\cup_{j=1}^{2} \mathcal{N}_{0}^{j}$ is a bifurcation associated to $\mathrm{P}_{1}$.

(iii) When $(\hat{\beta}, \hat{\delta})=(-1,1)$ there is an isolated stable equilibrium with coordinates $\mathrm{P}_{4}=(1,1,0)$ and a continuum of equilibria given by $\left(\left(2-\tilde{\tau}_{2}\right) \tilde{\tau}_{2}\right.$, $\left.\tilde{\tau}_{2}, 0\right)$ with $\tilde{\tau}_{2} \in(0,2)$. For these values of the parameters a degenerate bifurcation associated to $\mathrm{P}_{1}$ and $\mathrm{P}_{2}$ takes place. 
(iv) When $(\hat{\beta}, \hat{\delta})=(1,-1)$ there is an isolated stable equilibrium, namely, $\mathrm{P}_{3}=(-1,1,0)$ and a continuum of equilibria given by $\left(-\left(2-\tilde{\tau}_{2}\right) \tilde{\tau}_{2}, \tilde{\tau}_{2}, 0\right)$ with $\tilde{\tau}_{2} \in(0,2)$. For these values of the parameters $\mathrm{P}_{1}$ and $\mathrm{P}_{2}$ undergo a degenerate bifurcation.

Proof When $\sigma=0$ we get

$$
\mathcal{P}_{4}\left(\tilde{\tau}_{2} ; \sigma=0\right)=\tilde{\tau}_{2}^{2} \mathcal{R}_{2}\left(\tilde{\tau}_{2}\right)
$$

where

$$
\mathcal{R}_{2}\left(\tilde{\tau}_{2}\right)=\left[1+\hat{\beta}-(1-\hat{\delta}) \tilde{\tau}_{2}\right]\left[1-\hat{\beta}-(1+\hat{\delta}) \tilde{\tau}_{2}\right]
$$

The results are summarised in Fig. 5, where we have sketched the equilibria with their stability in each region of the parametric plane $(\hat{\beta}, \hat{\delta})$. White circles stand for saddles, equilibria with Poincaré index -1; black circles correspond to centres (index 1) and black and white circles stand for equilibria with index 0 .

The case $\tilde{\tau}_{2}=0$ has been already analysed. Thus, we are interested in the roots of $\mathcal{R}_{2}\left(\tilde{\tau}_{2}\right)$ such that $0<\tilde{\tau}_{2}<2$.

(a) From the first factor of $\mathcal{R}_{2}\left(\tilde{\tau}_{2}\right)$ we infer that

- $\tilde{\tau}_{2}=(1+\hat{\beta}) /(1-\hat{\delta})$ is a valid root of $\mathcal{R}_{2}\left(\tilde{\tau}_{2}\right)$ when $\hat{\delta} \neq 1$ and $0<$ $(1+\hat{\beta}) /(1-\hat{\delta})<2$, i.e. in $\mathcal{D}_{0} ;$ in $\mathcal{E}_{0}^{1} \cup \mathcal{N}_{0}^{1}$ with $\hat{\beta}>1 ; \mathcal{E}_{0}^{2}$ with $\hat{\beta}+2 \hat{\delta}<-1$; in $\mathcal{N}_{0}^{1}$ with $|\hat{\beta}|<1$; in $\mathcal{L}_{0}^{2}$ with $\hat{\delta}<0$; at $(1,-1)$. In this case, after replacing this value of $\tilde{\tau}_{2}$ in $\dot{\tilde{\tau}}_{3}=0$ in (75) we get the equilibrium

$$
\mathrm{P}_{3}=\left(\frac{(1+\hat{\beta})(1-\hat{\beta}-2 \hat{\delta})}{(1-\hat{\delta})^{2}}, \frac{1+\hat{\beta}}{1-\hat{\delta}}, 0\right) .
$$

The stability of this equilibrium is given by the non-trivial eigenvalues of matrix (63) which, once evaluated at $\mathrm{P}_{3}$ are

$$
\pm \frac{4 \sqrt{2} i}{L} \sqrt{\frac{(1+\hat{\beta})(\hat{\beta}+2 \hat{\delta}-1)}{\hat{\delta}-1}} .
$$

So, $\mathrm{P}_{3}$ is a saddle in $\mathcal{D}_{0}$ with $\hat{\beta}<-1$ and a centre otherwise. 


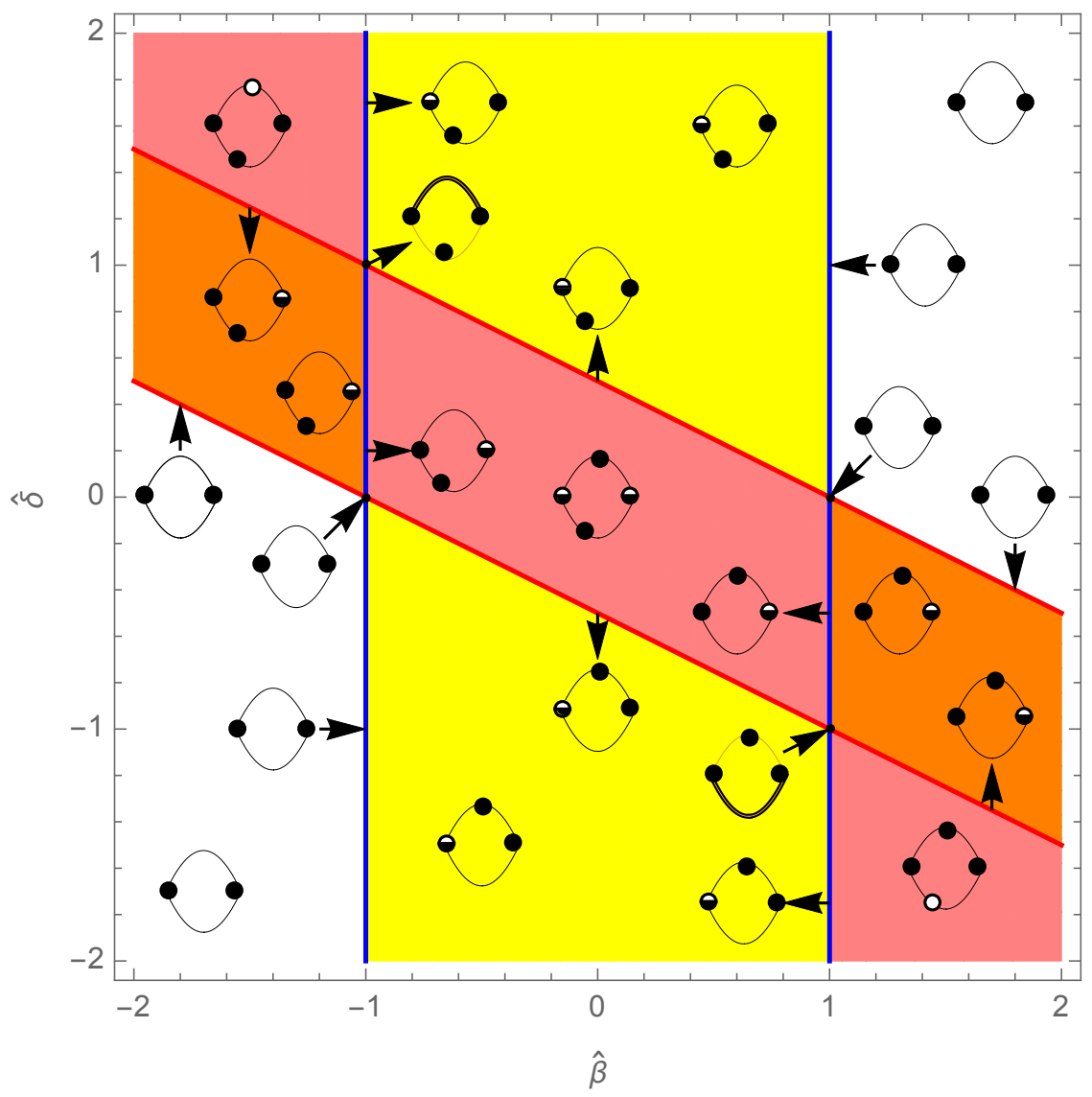

Figure 5. Bifurcation diagram when $\sigma=0$ and $\tilde{\alpha} \neq 0$. White regions correspond to $\cup_{j=1}^{2} \mathcal{C}_{0}^{j}$; pink regions represent $\mathcal{D}_{0}$; the orange ones stand for $\mathcal{E}_{0}^{1}$ and the yellow ones for $\mathcal{E}_{0}^{2}$. Blue lines are $\cup_{j=1}^{2} \mathcal{L}_{0}^{j}$ (bifurcations of $\mathrm{P}_{2}$ ) and the red ones are $\cup_{j=1}^{2} \mathcal{N}_{0}^{j}$ (bifurcations of $\mathrm{P}_{1}$ ). A sketch of the twice reduced space projected over $\tilde{\tau}_{3}=0$ together with the equilibria is represented for each parametric set. Black dots are stable points (index 1), white dots stand for saddles (index -1) and black and white circles represent equilibria with index 0 . At the point $(-1,1)$ the whole upper arc of the projection of $\mathcal{T}_{0}$ on $\tau_{3}=0$ are equilibria and at $(1,-1)$ it is the lower arc.

- When $(1+\hat{\beta}) /(1-\hat{\delta})=0$, i.e. $\hat{\beta}=-1$, and $\hat{\delta} \neq 1\left(\right.$ region $\left.\mathcal{L}_{0}^{1}\right)$ then, the equilibrium $\mathrm{P}_{3}$ collides with $\mathrm{P}_{2}$. Thus, there is a bifurcation associated to $\mathrm{P}_{2}$ that corresponds to the one appearing in Proposition 5.3 for $\tilde{\alpha} \neq 0$.

- When $(1+\hat{\beta}) /(1-\hat{\delta})=2$, i.e. $\hat{\beta}+2 \hat{\delta}=1$, and $\hat{\delta} \neq 1\left(\right.$ region $\left.\mathcal{N}_{0}^{2}\right)$ then, the equilibrium $\mathrm{P}_{3}$ collides with $\mathrm{P}_{1}$. Hence, there is a bifurcation 
associated to $\mathrm{P}_{1}$ that corresponds to the one appearing in Proposition 5.2 for $\tilde{\alpha} \neq 0$.

- When $\hat{\delta}=1$ and $\hat{\beta} \neq-1$ then, the first factor of $\mathcal{R}_{2}\left(\tilde{\tau}_{2}\right)$ does not give any root for the polynomial.

- When $\hat{\delta}=1$ and $\hat{\beta}=-1$ then $\mathcal{R}_{2}\left(\tilde{\tau}_{2}\right)$ trivially vanishes and there is a continuum of equilibria given by $\left(\left(2-\tilde{\tau}_{2}\right) \tilde{\tau}_{2}, \tilde{\tau}_{2}, 0\right)$ with $\tilde{\tau}_{2} \in(0,2)$. The point $(\hat{\beta}, \hat{\delta})=(-1,1)$ is the intersection of the lines $\hat{\beta}=-1$ (region $\mathcal{L}_{0}^{1}$, see Proposition 5.3 with $\tilde{\alpha} \neq 0$ ) and $\hat{\beta}+2 \hat{\delta}=1\left(\right.$ region $\mathcal{N}_{0}^{2}$, see Proposition 5.2 with $\tilde{\alpha} \neq 0$ ) and corresponds to a degenerate bifurcation associated to $\mathrm{P}_{1}$ and $\mathrm{P}_{2}$.

(b) We analyse the second factor of $\mathcal{R}_{2}\left(\tilde{\tau}_{2}\right)$ in the same way.

- The value $\tilde{\tau}_{2}=(1-\hat{\beta}) /(1+\hat{\delta})$ is a valid root of $\mathcal{R}_{2}\left(\tilde{\tau}_{2}\right)$ when $\hat{\delta} \neq-1$ and $0<(1-\hat{\beta}) /(1+\hat{\delta})<2$, i.e. in region $\mathcal{D}_{0}$; in $\mathcal{E}_{0}^{1} \cup \mathcal{N}_{0}^{2}$ with $\hat{\beta}<-1$; in $\mathcal{E}_{0}^{2}$ with $\hat{\beta}+2 \hat{\delta}>1$; in $\mathcal{N}_{0}^{2}$ with $|\hat{\beta}|<1$; in $\mathcal{L}_{0}^{1}$ with $\hat{\delta}>0$; and at $(\hat{\beta}, \hat{\delta})=(-1,1)$. In all cases, after substituting this value of $\tilde{\tau}_{2}$ in $\dot{\tilde{\tau}}_{3}=0$ in $(75)$ we get the equilibrium with coordinates

$$
\mathrm{P}_{4}=\left(-\frac{(1-\hat{\beta})(1+\hat{\beta}+2 \hat{\delta})}{(1+\hat{\delta})^{2}}, \frac{1-\hat{\beta}}{1+\hat{\delta}}, 0\right) .
$$

The stability of this equilibrium is given by the non-null eigenvalues of matrix (63) which, once evaluated at $\mathrm{P}_{4}$ are

$$
\pm \frac{4 \sqrt{2} i}{L} \sqrt{\frac{(1-\hat{\beta})(\hat{\beta}+2 \hat{\delta}+1)}{1+\hat{\delta}}} .
$$

Thus, $\mathrm{P}_{4}$ is a saddle in $\mathcal{D}_{0}$ with $\hat{\beta}>1$ and a centre otherwise.

- When $(1-\hat{\beta}) /(1+\hat{\delta})=0$, i.e. $\hat{\beta}=1$ and $\hat{\delta} \neq-1$ (region $\left.\mathcal{L}_{0}^{2}\right)$, the equilibrium $\mathrm{P}_{4}$ collides with $\mathrm{P}_{2}$. So, there is a bifurcation associated to $\mathrm{P}_{2}$ that corresponds to the one appearing in Proposition 5.3 for $\tilde{\alpha} \neq 0$. 
- When $(1-\hat{\beta}) /(1+\hat{\delta})=2$, i.e. $\hat{\beta}+2 \hat{\delta}=-1$ and $\hat{\delta} \neq-1\left(\right.$ region $\left.\mathcal{N}_{0}^{1}\right)$ the equilibrium $\mathrm{P}_{4}$ collides with $\mathrm{P}_{1}$. Thus, there is a bifurcation associated to $\mathrm{P}_{1}$ that corresponds to the one appearing in Proposition 5.2 for $\tilde{\alpha} \neq 0$.

- When $\hat{\delta}=-1$ and $\hat{\beta} \neq 1$ the second factor of $\mathcal{R}_{2}\left(\tilde{\tau}_{2}\right)$ does not give any root.

- When $\hat{\delta}=-1$ and $\hat{\beta}=1$ then $\mathcal{R}_{2}\left(\tilde{\tau}_{2}\right)$ trivially vanishes and there is a continuum of equilibria given by $\left(-\left(2-\tilde{\tau}_{2}\right) \tilde{\tau}_{2}, \tilde{\tau}_{2}, 0\right)$ with $\tilde{\tau}_{2} \in(0,2)$. The point $(\hat{\beta}, \hat{\delta})=(1,-1)$ is the intersection of the lines $\hat{\beta}=1$ (region $\mathcal{L}_{0}^{2}$, see Proposition 5.3 with $\tilde{\alpha} \neq 0$ ) and $\hat{\beta}+2 \hat{\delta}=-1$ (region $\mathcal{N}_{0}^{1}$, see Proposition 5.2 with $\tilde{\alpha} \neq 0$ ) and corresponds to a degenerate bifurcation associated to the points $\mathrm{P}_{1}$ and $\mathrm{P}_{2}$.

At this point the following sets are introduced:

$$
\begin{aligned}
& \mathcal{C}_{\sigma}^{1}=\left\{(\hat{\beta}, \hat{\delta}, \sigma) \in \mathbb{R}^{3} \mid \hat{\beta}+2 \hat{\delta}<-\sqrt{1-\sigma^{2}}, \hat{\beta}<1\right\} \\
& \mathcal{C}_{\sigma}^{2}=\left\{(\hat{\beta}, \hat{\delta}, \sigma) \in \mathbb{R}^{3} \mid \hat{\beta}+2 \hat{\delta}>\sqrt{1-\sigma^{2}}, \hat{\beta}>-1\right\} \\
& \mathcal{E}_{\sigma}=\left\{(\hat{\beta}, \hat{\delta}, \sigma) \in \mathbb{R}^{3}|| \hat{\beta}+2 \hat{\delta} \mid<\sqrt{1-\sigma^{2}}\right\} .
\end{aligned}
$$

Theorem 5.3. When $\tilde{\alpha} \neq 0$ and $\sigma \neq 0$ the two-parameter family defined by (74) on $\mathcal{T}_{\sigma}$ is structurally stable in the parametric region $\mathbb{R}^{2} \backslash\left\{\left(\cup_{j=1}^{2} \mathcal{N}^{j}\right) \cup \mathcal{L}^{\prime}\right\}$ (with $\mathcal{N}^{j}$ and $\mathcal{L}^{\prime}$ given in the paragraph before Theorem 5.2). There are three different situations:

- In the regions $\cup_{j=1}^{2} \mathcal{C}_{\sigma}^{j}$ and $\cup_{j=1}^{2} \mathcal{N}_{\sigma}^{j}$ with $\hat{\delta}(\hat{\beta}+2 \delta) \leq 1$, there are two stable equilibria, namely, $\mathrm{P}_{1}$ and a regular one.

- In these two cases there are three equilibria with the following stability features: 
(i) In the parametric sets $\cup_{j=1}^{2} \mathcal{N}_{\sigma}^{j}$ with $\hat{\delta}(\hat{\beta}+2 \delta)>1$ and $\mathcal{E}_{\sigma}$, the point $\mathrm{P}_{1}$ has Poincaré index zero and there are two stable equilibria at regular points.

(ii) In the region $\mathcal{L}^{\prime}$ the point $\mathrm{P}_{1}$ is stable, there is another stable equilibrium at a regular point and a third one with index 0.

- Otherwise there are four equilibria. The point $\mathrm{P}_{1}$ is stable and there are two other stable equilibria at regular points and one saddle at a regular point. Furthermore, the set $\mathcal{L}^{\prime}$ corresponds to a bifurcation of a regular equilibrium and $\cup_{j=1}^{2} \mathcal{N}^{j}$ represents a bifurcation of $\mathrm{P}_{1}$.

Proof We need to analyse the roots of $\mathcal{P}_{4}\left(\tilde{\tau}_{2}\right)$ such that $2|\sigma| \leq \tilde{\tau}_{2}<2$ and $\sigma \neq 0$. For that, we determine the values of the parameters where the number of valid roots of $\mathcal{P}_{4}\left(\tilde{\tau}_{2}\right)$ changes. Two possibilities arise:

(i) When a valid root of $\mathcal{P}_{4}\left(\tilde{\tau}_{2}\right)$ reaches the value $\tilde{\tau}_{2}=2$ then there is a collision of $\mathrm{P}_{1}$ (that is always at an equilibrium) with the equilibrium coming from the root of $\mathcal{P}_{4}\left(\tilde{\tau}_{2}\right)$. Thence, when

$$
\mathcal{P}_{4}(2)=4\left(1-\sigma^{2}\right)\left[1-\sigma^{2}-(\hat{\beta}+2 \hat{\delta})^{2}\right]=0
$$

there is a bifurcation involving $\mathrm{P}_{1}$. Recall that when $|\sigma|=1$, the space $\mathcal{T}_{\sigma}$ is a point, i.e. $\mathcal{T}_{-1}=\mathcal{T}_{1}=\{(0,2,0)\}$. So, we consider $|\sigma|<1$ and then, the parametric relation determining the bifurcation is $1-\sigma^{2}-(\hat{\beta}+$ $2 \hat{\delta})^{2}=0$, which defines $\cup_{j=1}^{2} \mathcal{N}_{\sigma}^{j}$ and corresponds to the one appearing in Proposition 5.2 for $\tilde{\alpha} \neq 0$. It is the red surface in Fig. 6 whose intersection with the plane $\sigma=0$ are the red lines in Fig. 5.

(ii) When there is a multiple root of $\mathcal{P}_{4}\left(\tilde{\tau}_{2}\right)$ associated to the same equilibrium then, there is a collision of equilibria coming from two valid roots of $\mathcal{P}_{4}\left(\tilde{\tau}_{2}\right)$. The occurrence of multiple roots of $\mathcal{P}_{4}\left(\tilde{\tau}_{2}\right)$ is given by $\mathcal{Q}_{6}(\sigma)$ in (76). Hence, when $\mathcal{Q}_{6}(\sigma)=0$ there is a multiple root of $\mathcal{P}_{4}\left(\tilde{\tau}_{2}\right)$. We discard the values of $\tilde{\tau}_{2}$ that do not lie in the interval $[2|\sigma|, 2)$ and those values corresponding 


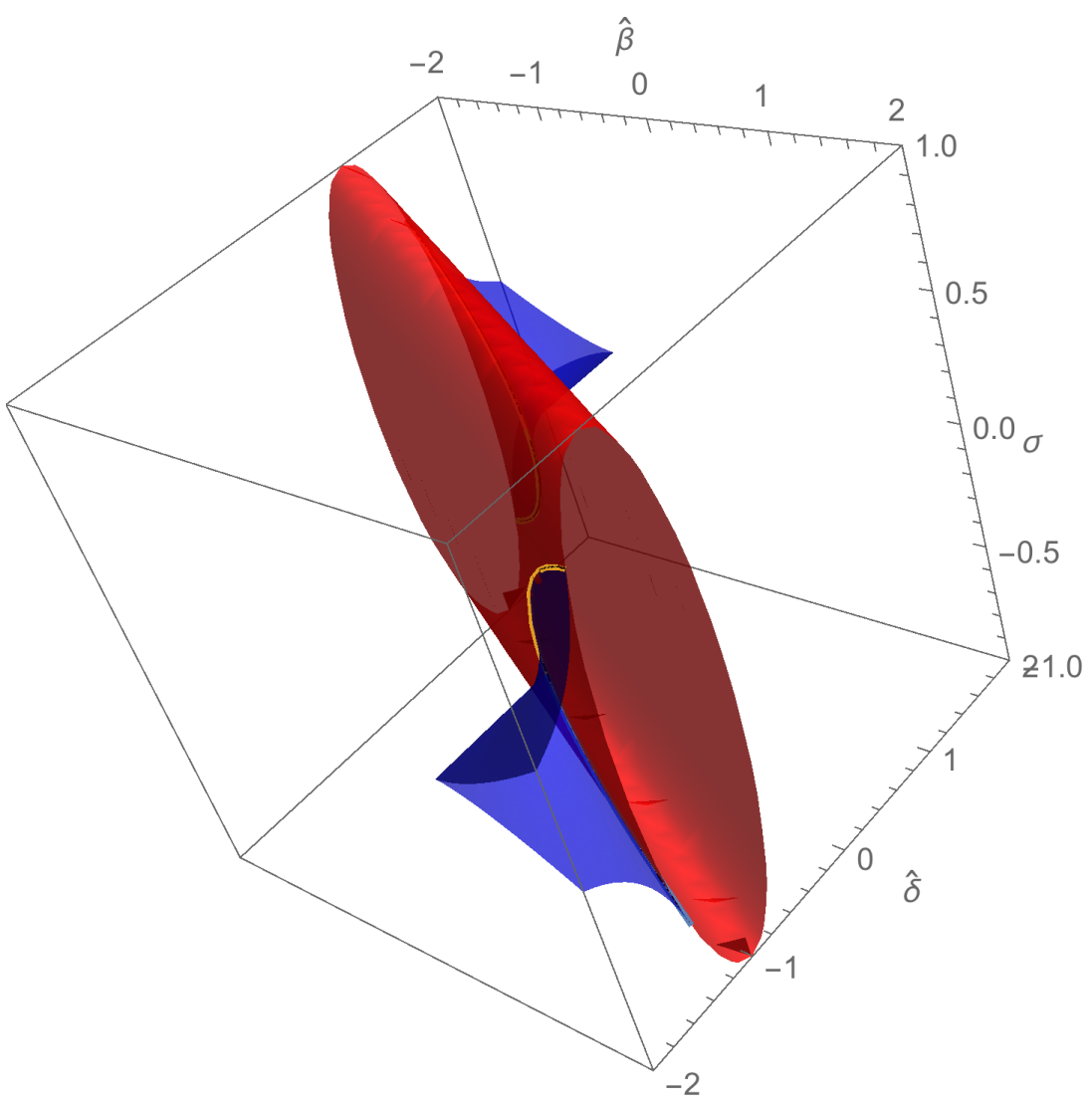

FiguRe 6. Bifurcation diagram for $\tilde{\alpha} \neq 0$. The red surface is $\cup_{j=1}^{2} \mathcal{N}_{\sigma}^{j}$ and represents a bifurcation associated to $\mathrm{P}_{1}$. The blue surface is $\mathcal{L}_{\sigma}^{\prime}$ and stands for a bifurcation at a regular point of $\mathcal{T}_{\sigma}$.

to more than one equilibrium. This gives us the blue surface appearing in

Fig. 6 that corresponds to $\mathcal{L}_{\sigma}^{\prime}$. In other words, $\mathcal{L}_{\sigma}^{\prime}$ represents a bifurcation associated to an equilibrium at a regular point of $\mathcal{T}_{\sigma}$. The expression $\hat{\delta}(\hat{\beta}+$ $2 \hat{\delta})=1$ comes from the intersection of $\mathcal{Q}_{6}(\sigma)=0$ and the relation appearing in (i), $1-\sigma^{2}-(\hat{\beta}+2 \hat{\delta})^{2}=0$, which is derived from $\mathcal{P}_{4}(2)=0$. It is represented by the yellow curve in Fig. 6. Note that the intersection of $\mathcal{Q}_{6}(\sigma)=0$ with the plane $\sigma=0$ is $\left(\hat{\beta}^{2}-1\right)^{3}=0$, that is, the set $\cup_{j=1}^{2} \mathcal{L}_{0}^{j}$ corresponds to the blue lines in Fig. 5 . 
In summary, $\cup_{j=1}^{2} \mathcal{N}_{\sigma}^{j}$ and $\mathcal{L}_{\sigma}^{\prime}$ divide the parametric space $(\hat{\beta}, \hat{\delta}, \sigma)$ into regions where the number of equilibria and the stability is maintained. An intersection of the bifurcation space with a plane $\sigma \neq 0$ is presented in Fig. 7. The picture presents for each region a sketch of $\mathcal{T}_{\sigma}$ projected over $\tilde{\tau}_{3}=0$ containing the equilibria and their stability.

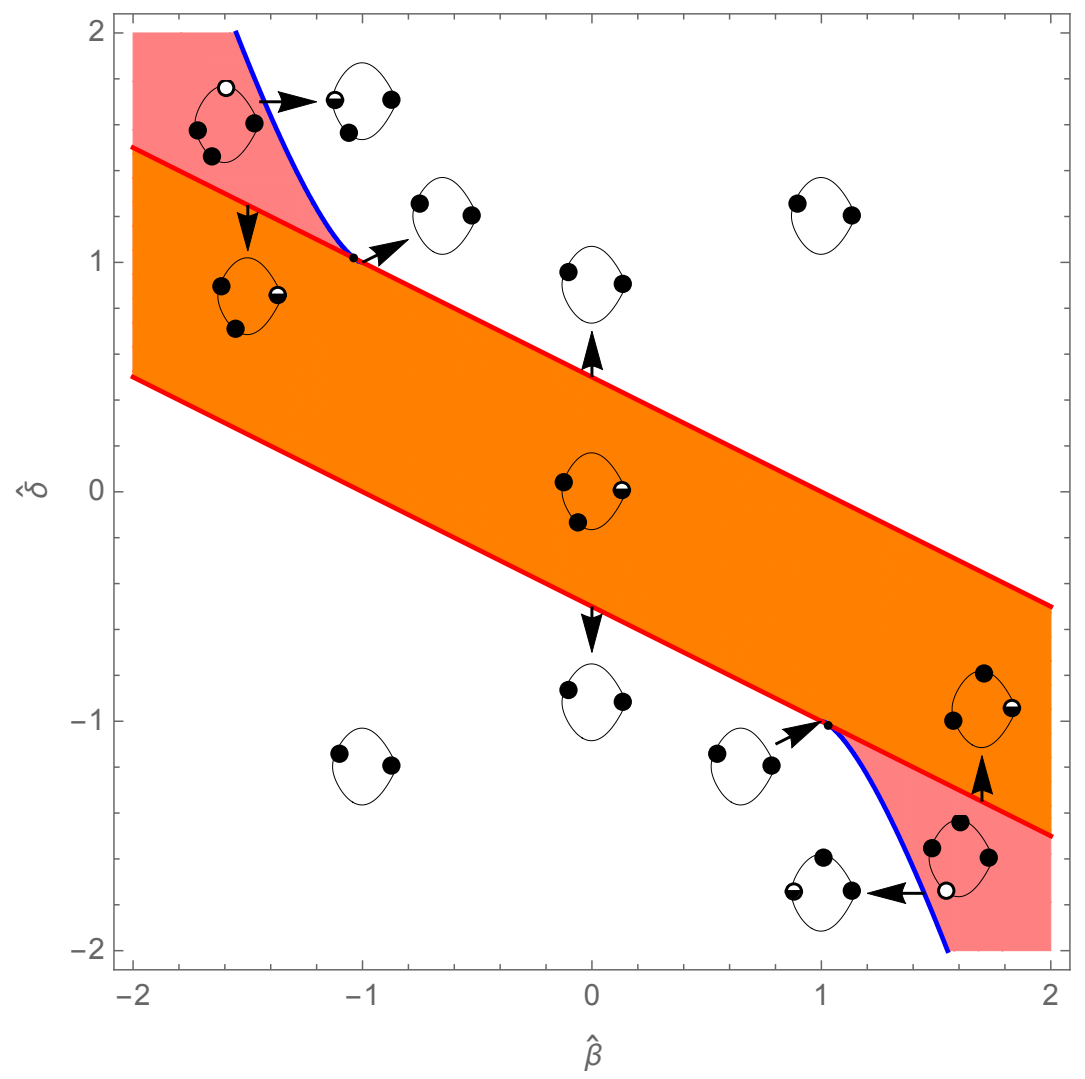

FiguRe 7. Intersection of the bifurcation diagram in the case $\tilde{\alpha} \neq$ 0 with the plane $\sigma \neq 0$ (the values in the picture are for $\sigma \sim 0$ ). A sketch of $\mathcal{T}_{\sigma}$ projected over $\tilde{\tau}_{3}=0$ together with the equilibria is represented for each parametric set. Black dots correspond to stable equilibria (index 1), white dots stand for saddles (index -1) and black and white circles represent equilibria with index 0 . The red lines correspond to $\cup_{j=1}^{2} \mathcal{N}_{\sigma}^{j}$ (a bifurcation of $\mathrm{P}_{1}$ ). The blue lines account for $\mathcal{L}^{\prime}$ (a bifurcation of a regular equilibrium). The white regions represent $\cup_{j=1}^{2} \mathcal{C}_{\sigma}^{j}$ and the orange region is $\mathcal{E}_{\sigma}$. The points of intersection of the blue and red lines correspond to the yellow curve in Fig. 6 . 
5.6. Bifurcations of equilibria. In this subsection we characterise the bifurcations appearing in the previous subsections. Basically we get

- Bifurcations of $\mathrm{P}_{1}$. They are given by the equation appearing in Proposition 5.2 , that is,

$$
|\tilde{\alpha}| \sqrt{1-\sigma^{2}}=|\beta+2 \tilde{\delta}|
$$

In Theorem 5.1 we have proved that when $\tilde{\alpha}=0$ this is a degenerate bifurcation. It corresponds to the set $\mathcal{N}_{\sigma}$, that is represented by the red surface in Fig. 3 and by the red lines in Fig. 4. In Theorem 5.2 we have proved that the case $\tilde{\alpha} \neq 0$ and $(\beta, \tilde{\delta}, \sigma) \in\{(-1 / \tilde{\alpha}, 1 / \tilde{\alpha}, 0),(1 / \tilde{\alpha},-1 / \tilde{\alpha}, 0)\}$ also corresponds to degenerate bifurcations of $\mathrm{P}_{1}$. So, here we consider $\tilde{\alpha} \neq 0$, $(\beta, \tilde{\delta}, \sigma) \notin\{(-1 / \tilde{\alpha}, 1 / \tilde{\alpha}, 0),(1 / \tilde{\alpha},-1 / \tilde{\alpha}, 0)\}$, in which case the bifurcation is represented by the set $\cup_{j=1}^{2} \mathcal{N}_{\sigma}^{j}$, that corresponds to the red surface in Fig. 6 and the red lines in Fig. 7. See Theorem 5.4 below for a more detailed description on the bifurcations occurring in $\cup_{j=1}^{2} \mathcal{N}_{\sigma}^{j}$.

- Bifurcations of $\mathrm{P}_{2}=(0,2|\sigma|, 0)$. On the one hand they are given by the equation appearing in Proposition 5.3, that is,

$$
|\tilde{\alpha}|=|\beta|
$$

In Theorem 5.1 we have proved that when $\tilde{\alpha}=0$ this bifurcation is degenerate. It corresponds to the set $\mathcal{L}_{\sigma}$, that is represented by the blue surface in Fig. 3 and by the blue line in Fig. 4. In Theorem 5.2 we have proved that the case $\tilde{\alpha} \neq 0$ and $(\beta, \tilde{\delta}, \sigma) \in\{(-1 / \tilde{\alpha}, 1 / \tilde{\alpha}, 0),(1 / \tilde{\alpha},-1 / \tilde{\alpha}, 0)\}$ also corresponds to degenerate bifurcations of $\mathrm{P}_{2}$. So, here we consider $\tilde{\alpha} \neq 0, \sigma=0$, $(\beta, \tilde{\delta}, \sigma) \notin\{(-1 / \tilde{\alpha}, 1 / \tilde{\alpha}, 0),(1 / \tilde{\alpha},-1 / \tilde{\alpha}, 0)\}$, in which case the bifurcation is represented by the set $\cup_{j=1}^{2} \mathcal{L}_{0}^{j}$, that corresponds to the blue lines in Fig. 5 .

On the other hand, when $\sigma=0$ and $-1<\hat{\beta}<1$, the point $\mathrm{P}_{2}=(0,0,0)$ corresponds to an equilibrium solution that disappears when $\sigma \neq 0$. This also occurs in [31], see for instance the passage through lines $A$ and $G$ of 
Fig. 3 in p. 342 of [31] with $\lambda$ fixed and $\mu$ passing from positive to negative.

- Bifurcations of regular equilibria. The set $\mathcal{L}_{\sigma}^{\prime}$ corresponds to a centre-saddle or Hamiltonian saddle-node bifurcation of a regular equilibrium, see [16]. It is the blue surface appearing in Fig. 6 or the blue lines in Fig. 7. On the blue surface an equilibrium with Poincaré index zero appears; in the pink region in Fig. 7 (and its associated in Fig. 6) it is transformed into a saddle and a centre; in the white region it disappears. See the evolution in Fig. 7.

Let us establish a result accounting for the bifurcations of the point $\mathrm{P}_{1}$. We introduce the Hamiltonian $\mathcal{K}_{\epsilon}$ as

$$
\mathcal{K}_{\epsilon}=\mathcal{K}+O\left(\epsilon^{2}\right),
$$

where $\mathcal{K}$ is given in (74).

Theorem 5.4. Given the two-parameter family defined by Hamiltonian $\mathcal{K}_{\epsilon}$ introduced in (77) on the space $\mathcal{T}_{\sigma}$, the set $\cup_{j=1}^{2} \mathcal{N}_{\sigma}^{j}$ corresponds to a Hamiltonian flip bifurcation of $\mathrm{P}_{1}$ and $\left(\cup_{j=1}^{2} \mathcal{N}_{\sigma}^{j}\right) \cap \mathcal{L}_{\sigma}^{\prime}$ corresponds to a degenerate Hamiltonian flip (i.e. a $\mathbb{Z}_{2}$-symmetric $A_{5}^{+}$) bifurcation of $\mathrm{P}_{1}$.

Proof We go back to the normal form around the blowup of $\mathrm{P}_{1}$ appearing in the proof of Proposition 5.2, that is formula (68) which, after rescaling and introducing the parameters $(\hat{\beta}, \hat{\delta})$ yields that

$$
\mathcal{K}(u, v)=e_{21} u^{2}+e_{22} v^{2}+e_{4}\left(u^{2}+v^{2}\right)^{2}+e_{6}\left(u^{2}+v^{2}\right)^{3}+\cdots,
$$

where

$$
\begin{aligned}
e_{21} & =\frac{\sqrt{1-\sigma^{2}}-\hat{\beta}-2 \hat{\delta}}{4 \sqrt{1-\sigma^{2}}}, & e_{22} & =-\frac{\sqrt{1-\sigma^{2}}+\hat{\beta}+2 \hat{\delta}}{4 \sqrt{1-\sigma^{2}}}, \\
e_{4} & =-\frac{\hat{\beta}+\hat{\delta}\left(1+\sigma^{2}\right)}{64\left(1-\sigma^{2}\right)^{2}}, & e_{6} & =-\frac{4(\hat{\beta}+\hat{\delta})+(\hat{\beta}+6 \hat{\delta}) \sigma^{2}}{2048\left(1-\sigma^{2}\right)^{7 / 2}} .
\end{aligned}
$$

- When $e_{22}=0$ then $e_{21}>0$. If $e_{4}>0$ a Hamiltonian flip bifurcation in the $\tau$-space associated to $\mathrm{P}_{1}$ takes place. Because of the 2:1 covering, 
this bifurcation is seen as a supercritical reversible Hamiltonian pitchfork bifurcation in the $(u, v)$-space. The same bifurcation appeared in [31]. This occurs on the part of the left half of the red surface in Fig. 6 that is not covered by the blue surface. This is the part of $\mathcal{N}_{\sigma}^{1}$ where $e_{4}>0$. In Fig. 7 it corresponds to the part of the bottom red line such that $\hat{\beta}<1$. We observe that $e_{21}$ and $e_{4}$ do not change sign when $e_{22} \approx 0$. The coefficient $e_{22}>0$ outside the red surface and $e_{22}<0$ inside the red surface. So, outside the red surface and on the red surface $(0,0)$ is stable and becomes a saddle and bifurcates giving rise to two centres when inside the red surface. In Fig. 7 we see that $P_{1}$ is stable in the white region and on the red line, it becomes a cusp in the orange region and a new stable equilibrium appears.

The same bifurcation occurs in the part of $\mathcal{N}_{\sigma}^{2}$ where $e_{4}<0$, that is, in the part of the right half red surface in Fig. 6 that is not covered by the blue surface or the top red line in Fig. 7 with $\hat{\beta}>-1$. In this case when $e_{21}=0, e_{22}<0$ and $e_{4}<0$.

- When $e_{22}=0$ and $e_{4}<0$ then, a Hamiltonian flip bifurcation occurs in the $\tau$-space. This time $\mathrm{P}_{1}$ becomes stable and gives rise to an unstable equilibrium. Due to the 2:1 covering, it is seen as a subcritical reversible Hamiltonian pitchfork bifurcation associated to $(0,0)$ that takes place in the $(u, v)$-space. This occurs on the part of the left half of the red surface in Fig. 6 that is covered by the blue surface. This is the part of $\mathcal{N}_{\sigma}^{1}$ where $e_{4}<0$. In Fig. 7 it corresponds to the part of the bottom red line such that $\hat{\beta}>1$. Again, $e_{21}$ and $e_{4}$ do not change sign when $e_{22} \approx 0$. The coefficient $e_{22}>0$ outside the red surface and $e_{22}<0$ inside the red surface, as we noticed before. So, inside the red surface and on this part of the red line, $(0,0)$ is a saddle with two associated centres. Once outside the red surface and below the blue one, it changes to a centre and two new saddles appear. In Fig. 7 we see that $P_{1}$ is stable in the pink region and there is also a 
saddle on a regular point. On the red line and in the orange region, $\mathrm{P}_{1}$ becomes a cusp and the saddle disappears.

The bifurcation just described happens in the part of $\mathcal{N}_{\sigma}^{2}$ with $e_{4}>0$, i.e. in the part of the right half red surface in Fig. 6 that is covered by the blue surface or the top red line in Fig. 7 with $\hat{\beta}<-1$. In this case when $e_{21}=0$, one has $e_{22}<0$ and $e_{4}>0$.

- When $e_{22}=0$ and $e_{4}=0$ then $\mathrm{P}_{1}$ undergoes a degenerate Hamiltonian flip bifurcation, see also [40] p. 36 . In the $(u, v)$-space it is seen as a $\mathbb{Z}_{2^{-}}$ symmetric $A_{5}^{+}$bifurcation of $(0,0)$. The diagram of the universal unfolding of this bifurcation can be seen in Fig. 2.6, p. 36 of [40]. In [64] this bifurcation is called a butterfly $A_{5}$ with reflection symmetry (see the sequence of bifurcations in Fig. 2, p. 4173). Here, this bifurcation occurs on the yellow line appearing in Fig. 6 that corresponds to the points $(1,-1)$ and $(-1,1)$ in Fig. 7. It represents the intersection of the red (Hamiltonian flip) and blue (centre-saddle) surfaces. This bifurcation also appears in [44]; see the intersection of lines $A$ and $B$ and around in Fig. 2, p. 94 for a representation in the $\tau$-space and compare with neighbourhoods of the points $(1,-1)$ and $(-1,1)$ in Fig. 7 here. In Fig. 8 we have depicted the flow of the reduced Hamiltonian around the point $(\hat{\beta}, \hat{\delta})=(1,-1)$, where the degenerate Hamiltonian flip bifurcation occurs.

We analyse the coefficients of the normal form on the yellow line with $\hat{\beta}>0$. An analogous analysis applies for the yellow line in Fig. 6 with $\hat{\beta}<0$.

(i) On the yellow line we get $e_{21}>0, e_{22}=0, e_{4}=0, e_{6}>0$. Hence, $(0,0)$ is a non-linear centre; see the sketch of $\mathcal{T}_{\sigma}$ corresponding to the point $(1,-1)$ in Fig. 7. 

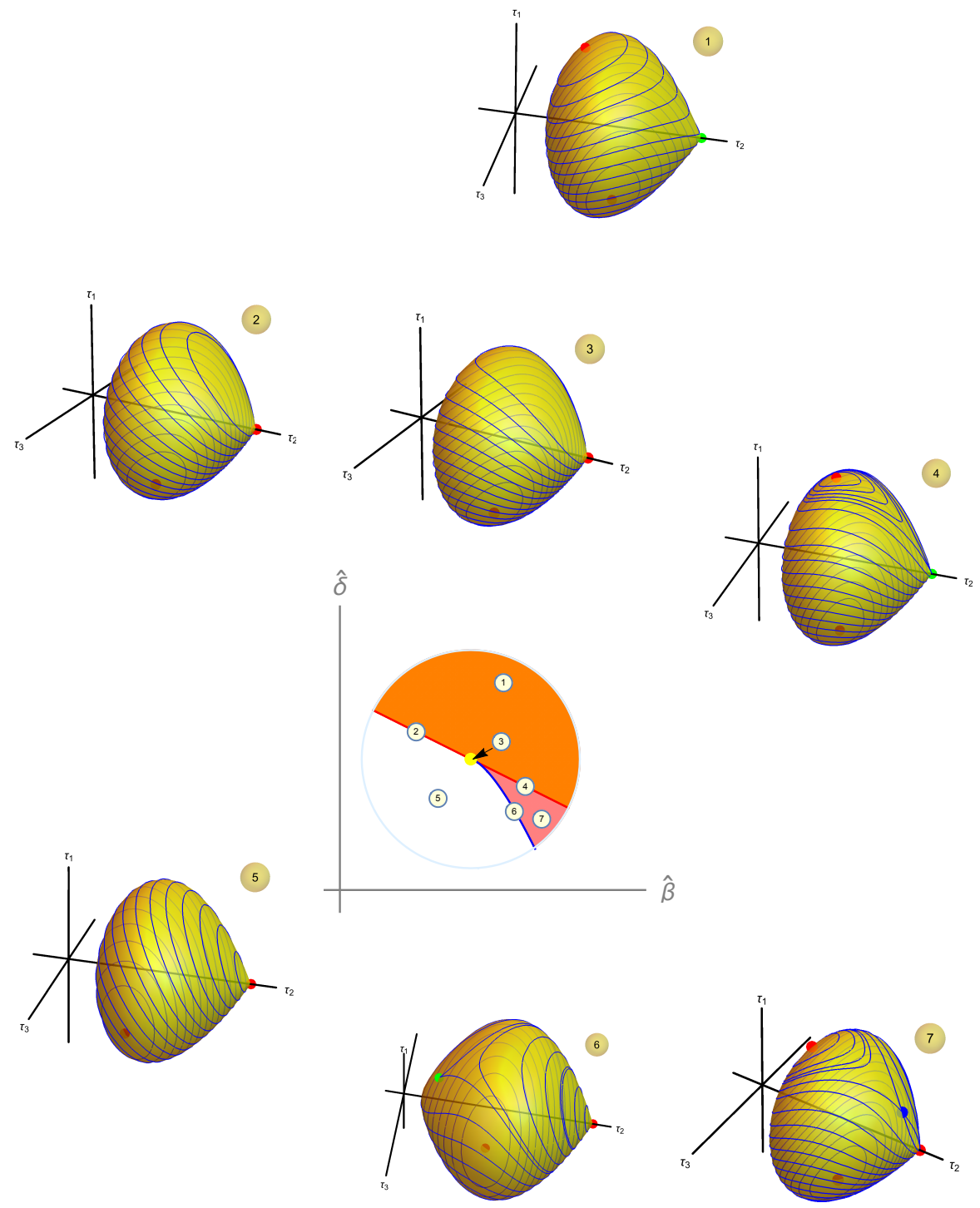

Figure 8. Zoom of Fig. 7 centered at $(\hat{\beta}, \hat{\delta})=(1,-1)$.

(ii) On the red surface that is not covered by the blue one the only change is that $e_{4}>0$. So, $(0,0)$ stays as a centre; see the sketch of $\mathcal{T}_{\sigma}$ corresponding to the part of the red bottom line with $\hat{\beta}<1$ in Fig. 7 .

(iii) On the part of the red surface that is covered by the blue one one has $e_{21}>0, e_{22}=0, e_{4}<0, e_{6}<0$. Thus, $(0,0)$ is now unstable and 
two centres bifurcate from it. This is the pitchfork bifurcation in the $(u, v)$ space that has already been seen. In the sketch of $\mathcal{T}_{\sigma}$ corresponding to the part of the red bottom line with $\hat{\beta}>1$ in Fig. 7, the pitchfork bifurcation becomes a Hamiltonian flip bifurcation, therefore we see one centre while the saddle is a cusp point.

(iv) Inside the red surface we have $e_{21}>0, e_{22}<0$ and the signs of $e_{4}$ and $e_{6}$ are not relevant. Then, $(0,0)$ continues to be a saddle with two associated centres; see the sketch of $\mathcal{T}_{\sigma}$ corresponding to the orange region in Fig. 7.

(v) Outside the red surface and below the blue one one gets $e_{21}>0$, $e_{22}>0, e_{4}<0$ and $e_{6}<0$. So, $(0,0)$ is now a centre and has two saddles associated to it to which two centres are attached. In the sketch of $\mathcal{T}_{\sigma}$ corresponding to the pink region in Fig. 7, apart from the peak, there is one centre and one saddle associated to it.

(vi) On the blue surface we see a bifurcation of the satellites which has been already studied, it is indeed the centre-saddle bifurcation analysed before. On this surface, $e_{4}^{2}-3 e_{21} e_{6} \approx 0$. It is exactly zero when we compute the Taylor series around the satellites. The centres and the saddles associated to the peak, which is stable, collide and form two degenerate equilibria. In the sketch of $\mathcal{T}_{\sigma}$ corresponding to the blue line in Fig. 7 we only see one degenerate point, i.e. the point which is being bifurcating.

(vii) Outside the blue surface the degenerate equilibria disappear. The peak is a centre as the coefficients $e_{21}, e_{22}$ are positive.

Now we deal with the bifurcations of $\mathrm{P}_{2}$. Specifically, we study the Hamiltonian Hopf bifurcations in the twice reduced space $\mathcal{T}_{\sigma}$ occurring for the singular equilibrium point $\mathrm{P}_{2}=(0,0,0)$. This bifurcation was analysed for the first time by Meyer and Schmidt [59], see also [54], in the setting of the planar circular restricted three body problem in the neighborhood of the equilibrium point $L_{4}$. Further 
studies applying singular reduction theory were developed by van der Meer [52]. More recently, Hanßmann and van der Meer [41, 42] have undertaken the study of the Hamiltonian Hopf bifurcation in the context of three-degrees-of-freedom systems, with special emphasis in the 1:1:1 resonance, see also [40]. Here we use an approach similar to Hanßmann and van der Meer but applying the symplectic coordinates introduced in Section 4 that are valid in the neighborhood of the point $\mathcal{O}_{R}=(0,2 h, 0,0,0,0,0,0,0)$ of Theorem 4.2. This is the point of the first reduced space $\mathbb{C P}_{h}^{2}$ that corresponds to the point $\mathrm{P}_{2}=(0,0,0)$ in the twice reduced space. We have to distinguish between non-degenerate and degenerate bifurcations.

Theorem 5.5. Given the two-parameter family defined by Hamiltonian (77) on the space $\mathcal{T}_{\sigma}$, the set $\cup_{j=1}^{2} \mathcal{L}_{0}^{j}$ corresponds to a Hamiltonian Hopf bifurcation of $\mathrm{P}_{2}$.

When $\hat{\delta}= \pm 1$ then, for $\epsilon$ sufficiently small, there is a smooth function $\hat{\beta}(\epsilon)$ close to $\mp 1$ such that Hamiltonian $\mathcal{K}_{\epsilon}=\mathcal{K}+\epsilon^{2} \mathcal{K}_{2}+O\left(\epsilon^{4}\right)$ with $\mathcal{K}_{2}$ corresponding to the normalised Hamiltonian of order 4 in $\epsilon$ once it has been twice reduced, undergoes a Hamiltonian Hopf bifurcation of $\mathrm{P}_{2}$ for certain combinations of the parameters.

Proof First of all, we consider the set $\cup_{j=1}^{2} \mathcal{L}_{0}^{j}$, thus either $\hat{\beta}=-1$ or $\hat{\beta}=1$. This is a bifurcation of the singular equilibrium point $\mathrm{P}_{2}=(0,0,0)$ which is equivalent to a bifurcation of the point $\mathcal{O}_{R}$ in $\mathbb{C P}_{h}^{2}$. Thus, we return to the reduced Hamiltonian given in (41) which is of course axially symmetric. Now, the four invariants associated with the axial symmetry written in terms of the regular coordinates $Q_{i}$, $P_{i}$ introduced in Theorem 4.2 are just

(78) $\Gamma_{1}=Q_{1} P_{2}-Q_{2} P_{1}, \quad \Gamma_{2}=\frac{1}{2}\left(Q_{1}^{2}+Q_{2}^{2}\right), \quad \Gamma_{3}=\frac{1}{2}\left(P_{1}^{2}+P_{2}^{2}\right), \quad \Gamma_{4}=Q_{1} P_{1}+Q_{2} P_{2}$,

with the constraint

$$
\Gamma_{1}^{2}+\Gamma_{4}^{2}=4 \Gamma_{2} \Gamma_{3}
$$

Notice that $\Gamma_{1}=-N$. 
The non-zero Poisson brackets are

$$
\begin{gathered}
\left\{\Gamma_{2}, \Gamma_{3}\right\}=-\left\{\Gamma_{3}, \Gamma_{2}\right\}=\Gamma_{4}, \quad\left\{\Gamma_{2}, \Gamma_{4}\right\}=-\left\{\Gamma_{4}, \Gamma_{2}\right\}=2 \Gamma_{2} \\
\left\{\Gamma_{4}, \Gamma_{3}\right\}=-\left\{\Gamma_{3}, \Gamma_{4}\right\}=2 \Gamma_{3} .
\end{gathered}
$$

In these invariants the reduced Hamiltonian (41) takes the simple form

$$
\begin{aligned}
\overline{\mathcal{H}}_{\epsilon}= & 2 \tilde{\alpha}(\hat{\beta}+1) \Gamma_{2}+2 \tilde{\alpha}(\hat{\beta}-1) \Gamma_{3}+\frac{2 \tilde{\alpha}}{h}\left[(\hat{\delta}-1) \Gamma_{2}^{2}+2 \hat{\delta} \Gamma_{2} \Gamma_{3}+(\hat{\delta}+1) \Gamma_{3}^{2}\right] \\
& -\frac{1}{9 h}\left[3 \tilde{\alpha}(\hat{\beta}+2 \hat{\delta}+2)+2 a_{2}\left(2 a_{2}-3 a_{4}\right) h\right] \Gamma_{1}^{2}+O\left(\epsilon^{2}\right),
\end{aligned}
$$

with $\tilde{\alpha}, \hat{\beta}$ and $\hat{\delta}$ introduced in (56). This expression has been obtained using the Gröbner basis relating the $\Gamma_{i}$ with $Q, P$, and replacing the coefficients $d_{i}, \omega_{i}$ of (41) in terms of $\tilde{\alpha}, \hat{\beta}, \hat{\delta}$ and $a_{j}$.

For $(\hat{\beta}, \hat{\delta}) \in \mathcal{L}_{0}^{1}$, that is, when $\hat{\beta}=-1$ and $\hat{\delta} \neq 1$ the coefficient of $\Gamma_{2}$ is zero while the coefficients of $\Gamma_{3}$ and $\Gamma_{2}^{2}$ are non-null. Then by virtue of Definition 2.24 of [40] we conclude that a Hamiltonian Hopf bifurcation takes place. Here, the signs of these coefficients determine the type of bifurcation. More precisely, Hamiltonian (77) with $\mathcal{K}$ introduced in (74) and obtained from (41) as it was described in the previous subsections of Section 5, undergoes a supercritical Hamiltonian Hopf bifurcation when $\hat{\delta}<1$ and a subcritical Hamiltonian Hopf bifurcation when $\hat{\delta}>1$. A supercritical bifurcation means that two families of periodic solutions emanate from the origin when $\hat{\beta}<-1$. These families persist when $\hat{\beta}=-1$ as two distinct families of periodic solutions. As $\hat{\beta}>-1$, the two families detach from the origin as a single family and move away from the origin. On the other hand, a subcritical Hamiltonian Hopf bifurcation means that two families emanate from the origin when $\hat{\beta}+1$ is small and negative, and the families are globally connected. This global family shrinks to the origin as $\hat{\beta}$ tends to zero. Finally when $\hat{\beta}+1$ becomes positive, there are no periodic solutions close to the origin. See also [54] and [41].

Proceeding in a similar way for the set $\mathcal{L}_{0}^{2}$ it is verified that Hamiltonian $\mathcal{K}_{\epsilon}$ undergoes a supercritical Hamiltonian Hopf bifurcation when $\hat{\delta}>-1$ and a subcritical Hamiltonian Hopf bifurcation when $\hat{\delta}<-1$. In this case the coefficient of 
$\Gamma_{3}$ in (79) becomes zero and one readily checks that the coefficients of $\Gamma_{2}$ and $\Gamma_{3}^{2}$ do not vanish.

We remark that the centre-saddle bifurcations of periodic solutions emanating from the subcritical Hamiltonian Hopf bifurcations correspond to the centre-saddle bifurcations of relative equilibria already studied in Theorem 5.2.

When $\hat{\delta}=1$ or $\hat{\delta}=-1$, a degenerate Hamiltonian Hopf bifurcation associated to $\mathrm{P}_{2}$ takes place. In order to analyse if the degeneration can be removed, we consider the next order in the reduced Hamiltonian(41), that is, we compute the normal form Hamiltonian of (6) up to sextic terms in the rectangular coordinates. After truncating higher order terms, putting the normalised Hamiltonian in terms of the $\rho_{i}$ and writing the resulting expression as a function of the rectangular coordinates $Q$ and $P$ of Theorem 4.2, we need to express it in terms of the $\Gamma_{j}$. After some manipulations, the reduced Hamiltonian is written as

$$
\overline{\mathcal{H}}_{\epsilon}=\Delta_{\epsilon}^{1} \Gamma_{2}+\Delta_{\epsilon}^{2} \Gamma_{3}+\frac{1}{h}\left(\Delta_{\epsilon}^{3} \Gamma_{2}^{2}+\Delta_{\epsilon}^{4} \Gamma_{2} \Gamma_{3}+\Delta_{\epsilon}^{5} \Gamma_{3}^{2}+\Delta_{\epsilon}^{6} \Gamma_{1}^{2}\right)+O\left(\epsilon^{4}\right),
$$


where

$$
\begin{aligned}
& \Delta_{\epsilon}^{1}=2 \tilde{\alpha}(\hat{\beta}+1)-\frac{\epsilon^{2}}{432} h^{2}\left[268 a_{5}^{4}+6852 a_{5}^{3} a_{10}+18 a_{5} a_{10}\left(957 a_{10}^{2}-485 b_{10}-762 b_{15}\right)\right. \\
& +3 a_{5}^{2}\left(1005 a_{10}^{2}-692 b_{10}-552 b_{15}\right)-27\left(2115 a_{10}^{4}+a_{10}^{2}\left(242 b_{10}-2700 b_{15}\right)\right. \\
& \left.\left.-17\left(b_{10}^{2}+4 b_{10} b_{15}-12 b_{15}^{2}\right)\right)\right] \\
& \Delta_{\epsilon}^{2}=2 \tilde{\alpha}(\hat{\beta}-1)-\frac{\epsilon^{2}}{432} h^{2}\left[76 a_{5}^{4}-1020 a_{5}^{3} a_{10}+18 a_{5} a_{10}\left(1203 a_{10}^{2}+43 b_{10}-1110 b_{15}\right)\right. \\
& -27\left(2115 a_{10}^{4}+a_{10}^{2}\left(238 b_{10}-2700 b_{15}\right)-b_{10}^{2}-28 b_{10} b_{15}+204 b_{15}^{2}\right) \\
& \left.+9 a_{5}^{2}\left(167 a_{10}^{2}+4\left(b_{10}+30 b_{15}\right)\right)\right] \\
& \Delta_{\epsilon}^{3}=2 \tilde{\alpha}(\hat{\delta}-1)-\frac{\epsilon^{2}}{144} h^{2}\left[672 a_{5}^{4}-4166 a_{5}^{3} a_{10}-6 a_{5} a_{10}\left(1914 a_{10}^{2}+138 b_{1}-797 b_{10}\right.\right. \\
& \left.-1524 b_{15}\right)-3 a_{5}^{2}\left(99 a_{10}^{2}+1016 b_{1}-46 b_{10}-160 b_{15}\right)+9\left(2115 a_{10}^{4}\right. \\
& \left.\left.+4 a_{10}^{2}\left(121 b_{10}-675 b_{15}\right)+17\left(4 b_{1} b_{10}-b_{10}^{2}-8 b_{10} b_{15}+12 b_{15}^{2}\right)\right)\right], \\
& \Delta_{\epsilon}^{4}=4 \tilde{\alpha} \hat{\delta}-\frac{\epsilon^{2}}{24}\left[100 a_{5}^{4}-588 a_{5}^{3} a_{10}+a_{5}^{2}\left(189 a_{10}^{2}-480 b_{1}+52 b_{10}-240 b_{15}\right)\right. \\
& -18 a_{5} a_{10}\left(240 a_{10}^{2}+24 b_{1}-29 b_{10}-208 b_{15}\right)+9\left(705 a_{10}^{4}+20 a_{10}^{2}\left(8 b_{10}-45 b_{15}\right)\right. \\
& \left.\left.+16 b_{1} b_{10}-3 b_{10}^{2}-32 b_{10} b_{15}+68 b_{15}^{2}\right)\right] \\
& \Delta_{\epsilon}^{5}=2 \tilde{\alpha}(\hat{\delta}+1)+\frac{\epsilon^{2}}{144} h^{2}\left[72 a_{5}^{4}-638 a_{5}^{3} a_{10}+6 a_{5} a_{10}\left(2406 a_{10}^{2}+294 b_{1}+275 b_{10}\right.\right. \\
& \left.-2220 b_{15}\right)-3 a_{5}^{2}\left(477 a_{10}^{2}+56 b_{1}+58 b_{10}-640 b_{15}\right)-9\left(2115 a_{10}^{4}\right. \\
& \left.\left.+4 a_{10}^{2}\left(119 b_{10}-675 b_{15}\right)+28 b_{1} b_{10}-b_{10}^{2}-56 b_{10} b_{15}+204 b_{15}^{2}\right)\right], \\
& \Delta_{\epsilon}^{6}=-\frac{1}{9}\left[3 \tilde{\alpha}(\hat{\beta}+2 \hat{\delta}+2)+2 a_{5}\left(2 a_{5}-3 a_{10}\right) h\right]+\frac{\epsilon^{2}}{432} h^{2}\left[220 a_{5}^{4}-1044 a_{5}^{3} a_{10}\right. \\
& +9 a_{5}^{2}\left(43 a_{10}^{2}-160 b_{1}+12 b_{10}-16 b_{15}\right)-18 a_{5} a_{10}\left(72 b_{1}-35 b_{10}\right) \\
& \left.+27 b_{10}\left(16 b_{1}+b_{10}\right)\right] \text {. }
\end{aligned}
$$

We have to look carefully at the coefficient $\Delta_{\epsilon}^{1}$, checking when it vanishes. When $\epsilon=0$ the unique solution to the equation $\Delta_{\epsilon}^{1}(\hat{\beta} ; 0)=0$ is $\hat{\beta}=-1$ and for this value it is verified that

$$
\Delta_{\epsilon}^{1}(-1 ; 0)=0, \quad \frac{\partial \Delta_{\epsilon}^{1}}{\partial \hat{\beta}}(-1 ; 0)=2 \tilde{\alpha} \neq 0
$$


Therefore, by the implicit function theorem there exists a unique solution $\hat{\beta}(\epsilon)$ of the equation $\Delta_{\epsilon}^{1}(\hat{\beta} ; \epsilon)=0$ satisfying $\hat{\beta}(0)=-1$. Next, in order to prove the nondegenerate condition, we compute $\hat{\beta}(\epsilon)$ in terms of $\epsilon$ up to order 2. After putting $b_{1}, b_{10}$ and $b_{15}$ in terms of $a_{5}, a_{10}, h, \tilde{\alpha}, \hat{\beta}$ and $\hat{\delta}$ we end up with

$$
\begin{aligned}
\hat{\beta}(\epsilon)=-1-\frac{\epsilon^{2} h}{54 \tilde{\alpha}}\left(2 a_{5}-3 a_{10}\right) & {\left[108\left(a_{5}+16 a_{10}\right) \tilde{\alpha}\right.} \\
+ & \left.35\left(2 a_{5}^{3}+15 a_{5}^{2} a_{10}-9 a_{5} a_{10}^{2}+54 a_{10}^{3}\right) h\right]+O\left(\epsilon^{4}\right) .
\end{aligned}
$$

Next, replacing this value in the coefficients $\Delta_{\epsilon}^{2}$ and $\Delta_{\epsilon}^{3}$, the relevant factor reads as

$$
\begin{aligned}
\Delta_{\epsilon}^{2} \Delta_{\epsilon}^{3}= & -\frac{16 \epsilon^{4} \tilde{\alpha}^{2}}{h}(\hat{\delta}-1)+\frac{16 \epsilon^{6} \tilde{\alpha}}{27 h}\left[549 \tilde{\alpha}^{2}(\hat{\delta}-1)\right. \\
- & 3 \tilde{\alpha}\left(27(31-2 \hat{\delta}) a_{10}^{2}+18(14 \hat{\delta}-69) a_{5} a_{10}+(80 \hat{\delta}+127) a_{5}^{2}\right) h \\
- & -5\left(567 a_{10}^{4}-9(\hat{\delta}-36) a_{5}^{2} a_{10}^{2}-27(\hat{\delta}+20) a_{5} a_{10}^{3}+4(2 \hat{\delta}+5) a_{5}^{4}\right. \\
& \left.\left.+3(8 \hat{\delta}-85) a_{5}^{3} a_{10}\right) h^{2}\right]+O\left(\epsilon^{8}\right) .
\end{aligned}
$$

Since the product $\Delta_{\epsilon}^{2} \Delta_{\epsilon}^{3}$ is generically non-zero for $\hat{\delta}=1$, by virtue of Definition 2.24 of [40] it follows that Hamiltonian $\mathcal{K}+O\left(\epsilon^{2}\right)$ with $\mathcal{K}$ given in (74) undergoes a Hamiltonian Hopf bifurcation at the value $\hat{\beta}(\epsilon)$. The bifurcation is of supercritical type when $\Delta_{\epsilon}^{2} \Delta_{\epsilon}^{3}>0$ and subcritical when the $\Delta_{\epsilon}^{2} \Delta_{\epsilon}^{3}<0$. We cannot conclude the occurrence of this bifurcation for the combinations of the parameters that make $\Delta_{\epsilon}^{2} \Delta_{\epsilon}^{3}$ vanish.

In the second case, proceeding similarly to the previous paragraphs, we have that

$$
\Delta_{\epsilon}^{2}(1 ; 0)=0, \quad \frac{\partial \Delta_{\epsilon}^{2}}{\partial \hat{\beta}}(1 ; 0)=2 \tilde{\alpha} \neq 0 .
$$

Thus, by the implicit function theorem there exists a unique solution $\hat{\beta}(\epsilon)$ of the equation $\Delta_{\epsilon}^{2}(\hat{\beta} ; \epsilon)=0$ satisfying $\hat{\beta}(0)=1$. The Taylor expansion of $\hat{\beta}(\epsilon)$ up to order 2 in $\epsilon$ is

$$
\hat{\beta}(\epsilon)=1+\frac{\epsilon^{2} h}{54 \tilde{\alpha}}\left(2 a_{5}-21 a_{10}\right)\left(a_{5}-3 a_{10}\right)\left[12 \tilde{\alpha}+5\left(2 a_{5}^{2}-9 a_{5} a_{10}+18 a_{10}^{2}\right) h\right]+O\left(\epsilon^{4}\right) .
$$


Replacing this value in the coefficients $\Delta_{\epsilon}^{2}$ and $\Delta_{\epsilon}^{3}$ we obtain

$$
\begin{aligned}
\Delta_{\epsilon}^{1} \Delta_{\epsilon}^{5}= & \frac{16 \epsilon^{4} \tilde{\alpha}^{2}}{h}(\hat{\delta}+1)-\frac{16 \epsilon^{6} \tilde{\alpha}}{27 h}\left[279 \tilde{\alpha}^{2}(\hat{\delta}+1)\right. \\
& +3 \tilde{\alpha}\left(9(6 \hat{\delta}+13) a_{10}^{2}-6(52 \hat{\delta}+55) a_{5} a_{10}+(28 \hat{\delta}+27) a_{5}^{2}\right) h \\
& +5\left(567 a_{10}^{4}-9(\hat{\delta}-30) a_{5}^{2} a_{10}^{2}-27(\hat{\delta}+26) a_{5} a_{10}^{3}+4(2 \hat{\delta}+3) a_{5}^{4}\right. \\
& \left.\left.+3(8 \hat{\delta}-9) a_{5}^{3} a_{10}\right) h^{2}\right]+O\left(\epsilon^{8}\right) .
\end{aligned}
$$

Now, since the above expression does not vanish generically, even for $\delta=-1$, by virtue of Definition 2.24 of [40], Hamiltonian $\mathcal{K}_{\epsilon}=\mathcal{K}+\epsilon^{2} \mathcal{K}_{2}+O\left(\epsilon^{4}\right)$ undergoes a Hamiltonian Hopf bifurcation at the value $\hat{\beta}(\epsilon)$. As before, the bifurcation is supercritical for $\Delta_{\epsilon}^{1} \Delta_{\epsilon}^{5}$ positive and subcritical when it is negative.

Remark 9. Specifying the values $a_{5}=3 \lambda a_{10}$ and $b_{1}=b_{10}=b_{15}=0$ in system (5), we obtain the axially symmetric Hamiltonian handled in [31]. Besides, it particularises to the 3D Hénon-Heiles Hamiltonian when $\lambda=-1$, see [32] and [33]. The resulting twice reduced Hamiltonian is as (79) with $\tilde{\alpha}, \hat{\beta}$ and $\hat{\delta}$ given by

$$
\tilde{\alpha}=-\frac{3}{4} a_{10}^{2} \lambda(6 \lambda-1) h, \quad \hat{\beta}=\frac{4 \lambda^{2}+6 \lambda-5}{\lambda(6 \lambda-1)}, \quad \hat{\delta}=-\frac{3 \lambda^{2}+12 \lambda-5}{2 \lambda(6 \lambda-1)} .
$$

In this context the sets $\mathcal{L}_{0}^{1}$ and $\mathcal{L}_{0}^{2}$ with $\tilde{\alpha}, \hat{\beta}$ and $\hat{\delta}$ as in (81) correspond to supercritical Hamiltonian Hopf bifurcations of $\mathrm{P}_{2}$ in the situations considered in [31] at the values $\lambda=1 / 2$ and $\lambda=5 / 2$; see [41] and [42] for more information.

When $\lambda=-1$ in (81) then $\hat{\beta}=-1$ and $\hat{\delta}=1$ and the Hamiltonian Hopf bifurcation becomes degenerate if only the normal form is considered up to terms in $O\left(\epsilon^{2}\right)$. The degeneracy is resolved by computing terms of order 4 in $\epsilon$. Instead of working with $\hat{\beta}$ and $\hat{\delta}$ one works with $\lambda$, obtaining, in a similar way as we had proceeded, a curve of the form $\lambda=-1+\epsilon^{2} \lambda^{*}+O\left(\epsilon^{4}\right)$. Then one concludes that a subcritical Hamiltonian Hopf bifurcation of $\mathrm{P}_{2}$ occurs for values of $\lambda$ close to -1 . It corresponds to a particular case of our treatment performed above. See [41] and references therein. 
Remark 10. The case $\hat{\beta}=1, \hat{\delta}=-1$ and $a_{5}=3 a_{10}$ corresponds to degenerate Hamiltonian Hopf bifurcations and we have checked that the degeneration remains adding higher order terms in the normal form (41) up to degree 10 in the rectangular coordinates $Q, P$. Moreover, using (81) it is easy to prove that this case has as a particular subcase the integrable family of axially symmetric Hamiltonians with $a_{5}=3 a_{10}$ and $b_{1}=b_{10}=b_{15}=0$, see [31] and references therein, because $\hat{\beta}=1$, $\hat{\delta}=-1$ imply $\lambda=1$.

Some studies $[20,28,29,70,34]$ have pointed out the relationship between integrability and the existence of a continuum of equilibria in the normal form. Indeed, the persistence of non-isolated equilibria through all the normal forms may be considered as an indicator of integrability, although there is not a rigorous proof of this fact. In this context, we conjecture that when $(\hat{\beta}, \hat{\delta})=(1,-1)$ and $a_{5}=3 a_{10}$, the family of Hamiltonians given by (5) is integrable. More specifically, this family becomes

$$
\mathcal{H}_{1}(q)=a_{10} z\left[3\left(x^{2}+y^{2}\right)+z^{2}\right], \quad \mathcal{H}_{2}(q)=b_{15}\left[\left(x^{2}+y^{2}\right)^{2}+6 z^{2}\left(x^{2}+y^{2}\right)+z^{4}\right] .
$$

\section{ReCOnstruction FROM the tWice REDUCED SPACE}

6.1. Invariant 3-tori related with elliptic points of $\mathcal{T}_{L, N}$. In this subsection we reconstruct the KAM tori of Hamiltonian (6) associated to the relative equilibria of the twice reduced system that are non-degenerate centres. For each relative equilibrium of the system defined by Hamiltonian (56) there is a Cantor family of KAM 3-tori for Hamiltonian function (6) provided the relative equilibrium is of elliptic type and non-degenerate. These equilibria can correspond to regular or singular points in the surface $\mathcal{T}_{L, N}$. As an example of a regular point, we choose $\mathrm{P}_{2}=(0,2|\sigma|, 0)$, with $0<|\sigma|<1$. This point is a relative equilibrium when $\tilde{\alpha}=0$ and is of centre type except for $2|\sigma| \tilde{\delta}+\beta=0$. An example of a singular point is $\mathrm{P}_{1}=(0,2,0)$, which is a stable point for $|\tilde{\alpha}| \sqrt{1-\sigma^{2}}<|\beta+2 \tilde{\delta}|$. In both cases we establish the existence of families of 3D KAM tori for the full system 
defined from (6). We do not consider the rest of the cases because the treatment is analogous.

Theorem 6.1. The following holds for the points $\mathrm{P}_{1}$ and $\mathrm{P}_{2}$ :

(i) Regarding the relative equilibrium $\mathrm{P}_{2}=(0,2|\sigma|, 0)$ with $0<|\sigma|<1$, when $\tilde{\alpha}=0,2|\sigma| \tilde{\delta}+\beta \neq 0$ and $\beta d_{5} \neq 0$, there are families of KAM 3-tori that are filled up by quasi-periodic solutions of Hamiltonian (6). These motions have an argument of pericentre $g$ near 0 , inclination with respect to the Oxy-plane near $\arccos (\sqrt{|\sigma| /(2-|\sigma|)})$ and eccentricity near $\sqrt{2(1-|\sigma|) /(2-|\sigma|)}$.

(ii) Regarding the relative equilibrium $\mathrm{P}_{1}=(0,2,0)$, when $|\beta+2 \tilde{\delta}| \neq|\tilde{\alpha}|$ and $d_{5} \neq 0$ then if $|\tilde{\alpha}| \sqrt{1-\sigma^{2}}<|\beta+2 \tilde{\delta}|$, there are families of KAM 3-tori filled up by near equatorial quasi-periodic solutions of the full system defined through Hamiltonian (6). When $N \approx 0$ these quasi-periodic motions are of near rectilinear-equatorial type.

In both cases the measure of the excluded set of the KAM tori is of order $O\left(\epsilon^{\mu}\right)$ with $0<\mu<1 / 5$.

Proof (i) As a set of action-angle coordinates to study the point $\mathrm{P}_{2}$ we choose nodal-Lissajous variables $(\ell, g, \nu, L, G, N)$, see [30]. Then, we start by expressing Hamiltonian (59) in these variables. The Hamiltonian depends on the coordinate $g$ and the momenta $(L, G, N)$. The coordinates of the equilibrium in the $(g, G)$ variables are $\left(g_{0}, G_{0}\right)=(0, \sqrt{(2 L-|N|)|N|})$. Then, in order to perform a local study of the Hamiltonian function around this equilibrium we introduce new coordinates $(\bar{g}, \bar{G})$ and apply the symplectic transformation with multiplier $\epsilon$ :

$$
g=\epsilon^{1 / 2} \bar{g}, \quad G=\sqrt{(2 L-|N|)|N|}+\epsilon^{1 / 2} \bar{G} .
$$

Then, we expand the Hamiltonian in powers of $\epsilon$, ending up with

$$
\epsilon \mathcal{K}(\bar{g}, \bar{G})=\frac{4|N|}{L}(\beta+2 \delta|N|)+\epsilon \mathcal{K}_{0}(\bar{g}, \bar{G})+\mathcal{O}\left(\epsilon^{2}\right)
$$


where

$$
\mathcal{K}_{0}(\bar{g}, \bar{G})=\frac{8(\beta+4 \delta|N|)(L-|N|)^{2}}{(2 L-|N|) L} \bar{g}^{2}+\frac{2(\beta+4 \delta|N|)(2 L-|N|)}{(L-|N|)^{2} L} \bar{G}^{2} .
$$

Now we define convenient action-angle variables $(\phi, J)$ with the aim of making $\mathcal{K}_{0}(\bar{g}, \bar{G})$ proportional to the action $J$. We set

$$
\bar{g}=\frac{\sqrt{J} \sqrt{2 L-|N|}}{L-|N|} \sin \phi, \quad \bar{G}=\frac{2 \sqrt{J}(L-|N|)}{\sqrt{2 L-|N|}} \cos \phi
$$

getting

$$
\epsilon \mathcal{K}(\phi, J)=\frac{4|N|}{L}(\beta+2 \delta|N|)+8 \epsilon \frac{J}{L}(\beta+4 \delta|N|)+\mathcal{O}\left(\epsilon^{2}\right)
$$

At this step we should reverse the operations that made possible the transformation from $\mathcal{H}_{\epsilon}$ to $\mathcal{K}$. First, we have rescaled time to pass from Hamiltonian (56) to Hamiltonian (59). So, we undo the rescaling by multiplying $\mathcal{K}(\phi, J)$ by $L / 2$. Now, Hamiltonian (56) is the expression of the twice reduced Hamiltonian after simplification of the constant terms and rescaling. So, we add these constant terms and rescale conveniently, arriving at

$$
\epsilon \mathcal{H}_{\epsilon}=L+\frac{\epsilon^{2}}{2}\left[4\left(d_{1}+d_{2}\right) L^{2}+d_{5} N^{2}\right]+\frac{\epsilon^{3}}{4} L \mathcal{K}(\phi, J)
$$

Next, we rescale time again getting

$\mathcal{H}_{\epsilon}=L+\frac{\epsilon^{2}}{2}\left[4\left(d_{1}+d_{2}\right) L^{2}+d_{5} N^{2}+2|N|(\beta+2 \delta|N|)\right]+2 \epsilon^{3}(\beta+4 \delta|N|) J+\mathcal{O}\left(\epsilon^{4}\right)$.

In terms of the $d_{i}$, taking into account that $\tilde{\alpha}=0$ implies $d_{4}=d_{3}$, Hamiltonian $\mathcal{H}_{\epsilon}$ reads as

$$
\mathcal{H}_{\epsilon}=h_{0}(L)+\epsilon^{2} h_{1}(L, N)+\epsilon^{3} h_{2}(L, N, J)+O\left(\epsilon^{4}\right)
$$

where

$$
\begin{aligned}
& h_{0}=L, \\
& h_{1}=\frac{1}{2}\left[4\left(d_{1}+d_{2}\right) L^{2}-\left(2 d_{3}-d_{5}\right) N^{2}-2\left(d_{2}-d_{3}\right) L|N|\right], \\
& h_{2}=-2\left[\left(d_{2}-d_{3}\right) L+2 d_{3}|N|\right] J .
\end{aligned}
$$


Now, we apply Han, Li, Yi's Theorem with $a=2, n_{0}=1, n_{1}=2, n_{2}=3, m_{1}=$ $2, m_{2}=3, y^{n_{0}}=L, y^{n_{1}}=(L, N), y^{n_{2}}=(L, N, J)$. The vector of frequencies has dimension 3 and is given by

$$
\Omega(L, N, J)=\left(1,2\left(2 d_{3}-d_{5}\right) N \mp 2\left(d_{2}-d_{3}\right) L,-2\left(d_{2}-d_{3}\right) L-4 d_{3}|N|\right) .
$$

Now we construct the $3 \times 4$ matrix whose columns are $\Omega, \partial \Omega / \partial L, \partial \Omega / \partial N$ and $\partial \Omega / \partial J$. When $\left(d_{2}-d_{3}\right) d_{5} \neq 0$ the rank of the matrix is 3 . (We stress that $d_{2} \neq d_{3}$ is equivalent to $\beta \neq 0$.) Therefore, Han-Li-Yi's Theorem guarantees the existence of KAM tori of dimension 3 . Setting $b=\sum_{j=1}^{a} m_{j}\left(n_{j}-n_{j-1}\right)$, we obtain $b=5$. The maximum order of the partial derivatives involved in the computation of the $3 \times 4$ matrix is $s=1$. As the perturbation is in an order $O\left(\epsilon^{4}\right)$ and $\epsilon^{s b+\mu}=\epsilon^{5+\mu}<\epsilon^{4}$, (for a pre-fixed small positive constant $\mu<1 / 5$ ), the excluding measure for the existence of the quasi-periodic invariant tori is of order $\epsilon^{\mu / s}=\epsilon^{\mu}$.

In order to obtain the geometric features of the quasi-periodic motions we take into account that the equilibrium point in nodal-Lissajous coordinates is $g_{0}=0$ and $G_{0}=\sqrt{(2 L-|N|)|N|}$, from where one deduces the inclination angle and the eccentricity of the trajectories. See more details in [30, 70].

(ii) Invariant 3-tori related with equatorial motions. Our goal is to establish the existence of KAM tori of dimension three associated to the singular equilibrium point $(0,2 L, 0)$ (or $\mathrm{P}_{1}=(0,2,0)$ in the coordinates $\tilde{\tau}_{1}, \tilde{\tau}_{2}$ and $\left.\tilde{\tau}_{3}\right)$ in the twicereduced $\mathcal{T}_{L, N}$. This point represents a family of equatorial orbits when $N \neq 0$ and a family of rectilinear equatorial orbits when $N=0$. In what follows we will proceed as in[56], first we find symplectic coordinates $x, y$ such that the normal form Hamiltonian (56) is written in terms of the new variables (and in terms of $L$ and $N$ ) and we can apply Han-Li-Yi's Theorem. As we are working in a neighborhood of the point $(0,2 L, 0)$ we introduce functions $f_{1}(x, y, L, N)$, and $f_{2}(x, y, L, N)$ to be 
determined such that the transformation

$$
\tau_{1}=f_{1}(x, y, L, N), \quad \tau_{2}=f_{2}(x, y, L, N), \quad \tau_{3}=\sqrt{\left(2 L-f_{2}\right)^{2}\left(f_{2}^{2}-4 N^{2}\right)-f_{1}^{2}},
$$

where we have used the constraint of (51) to put $\tau_{3}$ in terms of $f_{1}$ and $f_{2}$, be symplectic. Replacing the Poisson brackets between the $\tau_{i}$ given in Table 3 in terms of $x$ and $y$ and taking into account that we are imposing $\{x, y\}=1$ we build three partial differential equations (one for each Poisson bracket) whose unknowns are $f_{1}$ and $f_{2}$. Out of the three equations there is an essential expression that has to be zero, the other equations being redundant. The relevant equation is

$$
4 \sqrt{\left(2 L-f_{2}\right)^{2}\left(f_{2}^{2}-4 N^{2}\right)-f_{1}^{2}}+\frac{\partial f_{1}}{\partial x} \frac{\partial f_{2}}{\partial y}-\frac{\partial f_{1}}{\partial y} \frac{\partial f_{2}}{\partial x}=0
$$

Next, we select a convenient value for $f_{2}$. In fact we take $f_{2}=2 L-x^{2}-y^{2}$ and solve for $f_{1}$, getting

$$
f_{1}(x, y, L, N)=2 x y \sqrt{\left(2 L-x^{2}-y^{2}\right)^{2}-4 N^{2}} .
$$

We note that $x=y=0$ implies $f_{1}=0, f_{2}=2 L$ and $\tau_{1}=\tau_{3}=0$ while $\tau_{2}=2 L$. Once $f_{1}$ and $f_{2}$ are calculated the final change is

$$
\begin{aligned}
& \tau_{1}=2 x y \sqrt{\left(2 L-x^{2}-y^{2}\right)^{2}-4 N^{2}}, \\
& \tau_{2}=2 L-x^{2}-y^{2}, \\
& \tau_{3}=\left(y^{2}-x^{2}\right) \sqrt{\left(2 L-x^{2}-y^{2}\right)^{2}-4 N^{2}} .
\end{aligned}
$$

The equation $\tau_{2}=2 L-x^{2}-y^{2}$ represents the local regular surface around the equilibrium $x=y=0$ in the space $x y \tau_{2}$, which means that $(0,2 L, 0)$ is a regular point in this chart. In particular when $N=0$ the above transformation reduces to

$$
\tau_{1}=2 x y\left(2 L-x^{2}-y^{2}\right), \quad \tau_{2}=2 L-x^{2}-y^{2}, \quad \tau_{3}=\left(y^{2}-x^{2}\right)\left(2 L-x^{2}-y^{2}\right) .
$$

Now, Hamiltonian (56) is put in terms of $x$ and $y$ using (82). Next we scale $x$ and $y$ by doing $(x, y)=\epsilon^{1 / 2}(\bar{x}, \bar{y})$, multiply $\mathcal{K}$ by the multiplier $\epsilon^{-1}$ and Taylor expand 
the result around $\epsilon=0$. One obtains

$$
\epsilon \mathcal{K}(\bar{x}, \bar{y})=4(\beta+2 \delta L)-\frac{2 \epsilon}{L}\left[(\beta+4 \delta L)\left(\bar{x}^{2}+\bar{y}^{2}\right)-4 \alpha \sqrt{L^{2}-N^{2}} \bar{x} \bar{y}\right]+O(\epsilon)
$$

At this point we should prepare the Hamiltonian to apply a KAM-type theorem. For this purpose, we introduce adequate action-angle variables $(\varphi, J)$. After some manipulations we define the transformation:

$$
\begin{aligned}
\bar{x}= & -2^{1 / 2} \sqrt{\frac{|\beta+4 \delta L| J}{\sqrt{\beta^{2}+8 \beta \delta L+4\left[4 \delta^{2} L^{2}+\alpha^{2}\left(N^{2}-L^{2}\right)\right]}}} \cos \varphi, \\
\bar{y}= & \mp 2^{3 / 2} \alpha \sqrt{\frac{\left(L^{2}-N^{2}\right) J}{|\beta+4 \delta L| \sqrt{\beta^{2}+8 \beta \delta L+4\left[4 \delta^{2} L^{2}+\alpha^{2}\left(N^{2}-L^{2}\right)\right]}}} \cos \varphi \\
& +2^{1 / 2} \sqrt{\frac{\sqrt{\beta^{2}+8 \beta \delta L+4\left[4 \delta^{2} L^{2}+\alpha^{2}\left(N^{2}-L^{2}\right)\right]} J}{|\beta+4 \delta L|}} \sin \varphi,
\end{aligned}
$$

where the upper sign applies for $\beta+4 \delta L>0$ and the lower sign applies for $\beta+4 \delta L<$ 0 . Since the restriction $|\tilde{\alpha}| \sqrt{1-\sigma^{2}}<|\beta+2 \tilde{\delta}|$ is equivalent to $\beta^{2}+8 \beta \delta L+4\left[4 \delta^{2} L^{2}+\right.$ $\left.\alpha^{2}\left(N^{2}-L^{2}\right)\right]>0$, this change is well defined. Thus the Hamiltonian (56) in the action-angle coordinates becomes

$$
\epsilon \mathcal{K}(\phi, J)=4(\beta+2 \delta L) \mp 4 \epsilon \frac{J}{L} \sqrt{\beta^{2}+8 \beta \delta L+4\left[4 \delta^{2} L^{2}+\alpha^{2}\left(N^{2}-L^{2}\right)\right]}+O\left(\epsilon^{2}\right) .
$$

In the next step we should reverse the operations that passed from $\mathcal{H}_{\epsilon}$ to $\mathcal{K}$ and express the Hamiltonian in action-angle type coordinates. So, we undo the rescaling by multiplying $\mathcal{K}(\phi, J)$ by $L / 2$, add $4\left(d_{1}+d_{2}\right) L^{2}+d_{5} N^{2}$ and rescale time by multiplying the Hamiltonian by $\epsilon^{2} / 2$ and add $L$. The resulting Hamiltonian is

$$
\epsilon \mathcal{H}_{\epsilon}=L+\frac{\epsilon^{2}}{2}\left[4\left(d_{1}+d_{2}\right) L^{2}+d_{5} N^{2}\right]+\frac{\epsilon^{3}}{4} L \mathcal{K}(\phi, J)
$$

Finally, we rescale time again and replace the values of the parameters $\alpha, \beta$ and $\delta$ in terms of the $d_{i}$, getting

$$
\mathcal{H}_{\epsilon}=h_{0}(L)+\epsilon^{2} h_{1}(L, N)+\epsilon^{3} h_{2}(L, N, J)+O\left(\epsilon^{4}\right)
$$


where

$$
\begin{aligned}
& h_{0}=L, \\
& h_{1}=\frac{1}{2}\left[2\left(2 d_{1}+d_{2}\right) L^{2}+d_{5} N^{2}\right], \\
& h_{2}=\mp \frac{1}{2} \sqrt{4\left(d_{2}+d_{3}\right)\left(d_{2}+d_{4}\right) L^{2}+\left(d_{3}-d_{4}\right) N^{2}} J .
\end{aligned}
$$

Now we apply Han-Li-Yi's Theorem with $a=2, m_{1}=2, m_{2}=3, n_{0}=1, n_{1}=2$, $n_{2}=3, y^{n_{0}}=\hat{y}^{n_{0}}=L, y^{n_{1}}=(L, N), y^{n_{2}}=(L, N, J), \hat{y}^{n_{1}}=N$ and $\hat{y}^{n_{2}}=J$. One gets

$$
\Omega(L, N, J)=\left(1,-d_{5} N, \mp \frac{1}{2} \sqrt{4\left(d_{2}+d_{3}\right)\left(d_{2}+d_{4}\right) L^{2}+\left(d_{3}-d_{4}\right)^{2} N^{2}}\right)
$$

and for $d_{5} \neq 0, d_{2}+d_{3} \neq 0$ and $d_{2}+d_{4} \neq 0$, the matrix of order $3 \times 4$ whose rows are $\Omega, \partial \Omega / \partial L, \partial \Omega / \partial N$ and $\partial \Omega / \partial J$ has rank three, leading to the existence of the invariant tori of dimension three. According to [39] the excluding measure for the existence of these invariant tori is of order $O\left(\epsilon^{\mu}\right)$ with $0<\mu<1 / 5$. Notice that $\left(d_{2}+d_{3}\right)\left(d_{2}+d_{4}\right)=\tilde{\alpha}^{2}-(\beta+2 \tilde{\delta})^{2}$ from where one deduces that $\left(d_{2}+d_{3}\right)\left(d_{2}+d_{4}\right) \neq 0$ is the same as $|\tilde{\alpha}| \neq|\beta+2 \tilde{\delta}|$.

The computations carried out are valid for all $N$, thus we can conclude that the KAM 3-tori also exist for $N$ near zero.

6.2. Dynamics on the full Hamiltonian obtained from $\mathcal{T}_{L, N}$. Some information on the dynamics of Hamiltonian system associated to (6) has been obtained from the analyses performed in the spaces $\mathcal{M}_{\gamma}$ and $\mathbb{C P}_{h}^{2}$, in particular about the existence of periodic solutions and KAM 3-tori. Now we state a result regarding the dynamics of (6) related with the reconstruction from the twice reduced space.

Theorem 6.2. Given the parametric Hamiltonian (6), for a sufficiently small $\epsilon>$ 0 , we get:

(i) The relative equilibria of the system defined by Hamiltonian (56) give rise to families of invariant 2-tori in $T^{*} \mathbb{R}^{3}$ of the Hamiltonian system associated to (6) except the equilibrium point $(0,0,0)$ which leads to families of periodic solutions that are close to rectilinear in the OzZ-plane. In case of 
non-degeneracy the linear stability character of the 2-tori (or the rectilinear periodic solutions) coincides with the stability of the equilibria they come from.

(ii) The bifurcations of the relative equilibria of Hamiltonian (56) give rise to bifurcations involving families of invariant 2-tori (and some periodic solutions when the equilibrium point is $\left.\mathrm{P}_{2}=(0,0,0)\right)$ for the system related to Hamiltonian (6).

(iii) The elliptic relative equilibria of the system defined by Hamiltonian (56) give rise to Cantor families of KAM 3-tori for Hamiltonian function (6) provided they are of non-degenerate nature.

\section{Proof}

(i) We could have performed the normalisation process of Section 4 working directly in $\mathcal{M}_{\gamma}$ with the invariants $i_{j}$, transforming Hamiltonian (10) into normal form and reducing it again, after truncation of higher order, leading also to Hamiltonian (56) since the diagram 1 commutes. This was indeed done in [31]. Then, by Reeb's Theorem [62, 71], non-degenerate critical points of $\mathcal{T}_{L, N}$ are reconstructed as families of periodic solutions in $\mathcal{M}_{\gamma}$ provided the non-degeneracy conditions on the relative equilibria in Section 5 hold. This is also satisfied for the origin of the lemon, i.e. the point $\mathrm{P}_{2}=$ $(0,0,0)$, which is reconstructed as a family of periodic solutions of rectilinear polar type, which is realised in the points of the form $\left(0,0,0, i_{5}, i_{6}\right)$ of $\mathcal{M}_{0}$. Note that indeed it is the subspace of singular points of $\mathcal{M}_{\gamma}$. Finally, in all cases, the linear stability character of the periodic solutions is inherited from the (linear) stability of the critical points when the eigenvalues of their corresponding linearisations do not vanish.

Next, by undoing the axial symmetry reduction, each family of periodic solutions in $\mathcal{M}_{\gamma}$, except for the ones related to $\left(0,0,0, i_{5}, i_{6}\right)$ (where in 
addition $\gamma=0$ ), is transformed into a family of tori of dimension 2 in $T^{*} \mathbb{R}^{3}$. The linear stability of the invariant 2 -tori is the same as the linear stability of the periodic solution in $\mathcal{M}_{\gamma}$. On the other hand, the points in the singular subspace of $\mathcal{M}_{\gamma}$ are reconstructed to rectilinear motions in the $O z Z$-plane in the space $T^{*} \mathbb{R}^{3}$. In this case one obtains periodic solutions nearly rectilinear for Hamiltonian (6), because of the truncation of higher order terms due to the normal form computation.

Alternatively we can reconstruct the point $\mathrm{P}_{2}=(0,0,0)$ passing firstly to the space $\mathbb{C P}_{2}(h)$. Indeed its antiimage of the axial symmetry map is the point $\mathcal{O}_{R}$ since the passage from $\mathbb{C P}_{2}(h)$ to $\mathcal{T}_{L, N}$ fixes the $O z Z$-plane. Thus, we apply Reeb's Theorem to $\mathcal{O}_{R}$, getting families of periodic solutions that are nearly rectilinear for the Hamiltonian (6). This is indeed Theorem 4.2.

(ii) There is only one bifurcation that exclusively involves regular points in $\mathcal{T}_{L, N}$. It is the centre-saddle or Hamiltonian saddle-node bifurcation corresponding to the blue surface in Figure 6, see Section 5.5). A centre-saddle bifurcation associated to non-degenerate critical points of $\mathcal{T}_{L, N}$ is reconstructed to a centre-saddle bifurcation of families of periodic orbits in $\mathcal{M}_{\gamma}$ and to a quasi-periodic centre-saddle bifurcation of families of 2-tori in $T^{*} \mathbb{R}^{3}$, see $[16,57]$.

The Hamiltonian flip bifurcations established in Theorem 5.4 are reconstructed as period-doubling bifurcations in $\mathbb{C P}_{2}(h)$ : a periodic solution becomes unstable (or stable) and gives rise to a stable (or unstable) periodic solution of twice its period. They are quasi-periodic Hamiltonian flip (or frequency halving) bifurcations involving invariant 2 -tori in $T^{*} \mathbb{R}^{3}$ for the full system. The description of them is the same as the one made in [31]. The degenerate case of this bifurcation, that we have found in the region 
$\left(\cup_{j=1}^{2} \mathcal{N}_{\sigma}^{j}\right) \cap \mathcal{L}_{\sigma}^{\prime}$, is reconstructed as a degenerate quasi-periodic Hamiltonian flip bifurcation of invariant 2-tori in $T^{*} \mathbb{R}^{3}$, see more details in [40].

Supercritical and subcritical non-degenerate and degenerate Hamiltonian Hopf bifurcations take place in $\mathcal{T}_{L, N}$, see Theorem 5.5. When reconstructing to the full Hamiltonian (6), for the interpretation of the occurring bifurcations, one replaces the families of periodic solutions and their stability by families of invariant 2-tori and periodic solutions (when they are related to the point $\mathrm{P}_{2}$ ) and their stability. See also the descriptions made in $[41,42]$. We stress that the last step to recover the bifurcations involving the 2-tori is straightforward because the axial symmetry is exact and no truncation of higher-order terms is needed at this point. This makes its analysis a bit easier than that made by Broer et al [5].

(iii) This is indeed Theorem 6.1 and we have proved the existence of KAM 3-tori in two cases, the remaining cases being similar.

\section{ON THE DYNAMICS OF NON-AXIALLY SYMMETRIC HAMILTONIAN SYSTEMS}

So far we have studied the case of an isotropic 3D Hamiltonian with axially symmetric perturbations. However we can apply some of the results of the previous sections to perturbations of the type (4) provided the parameters $a_{i}, b_{i}$ satisfy some conditions. The main result in this direction is collected in the following theorem, where we establish the existence of many quasi-periodic solutions for non-axially symmetric perturbations of Hamiltonian systems in 1:1:1 resonance.

Theorem 7.1. Given the parametric Hamiltonian (6) where the higher order terms are (4), for a sufficiently small $\epsilon>0$ :

(i) Whenever the parameters $a_{i}$ and $b_{i}$ satisfy the conditions of Table 4 , then Hamiltonian $\mathcal{H}_{\epsilon}$ can be reduced by the oscillator and the axial symmetries 
up to terms of order 2 in $\epsilon$ and the corresponding twice reduced Hamiltonian can be written as

$$
\mathcal{K}_{\epsilon}=\alpha^{*} \tau_{1}+\beta^{*} \tau_{2}+\delta^{*} \tau_{2}^{2}+O\left(\epsilon^{2}\right)
$$

for some coefficients $\alpha^{*}, \beta^{*}$ and $\delta^{*}$ that depend on $L, N, a_{j}$ and $b_{k}$. Thus, the results established in Section 5 apply to Hamiltonian system (83) after replacing $\alpha$ by $\alpha^{*}, \beta$ by $\beta^{*}$ and $\delta$ by $\delta^{*}$.

(ii) Associated to each relative equilibrium of the system defined by Hamiltonian (83) there is a family of KAM 3-tori for Hamiltonian function (6) with perturbation (4) provided the relative equilibrium is of elliptic type and nondegenerate.

Proof To prove (i) one starts by normalising the Hamiltonian related to (6) and (4) up to quartic terms in the coordinates $q=(x, y, z)$ and $p=(X, Y, Z)$, that is, by performing two steps in the Lie-Deprit method. The resulting Hamiltonian does not enjoy the axial symmetry, in general. Then we compute the Poisson bracket of the normalised quartic terms and $x Y-y X$, yielding a homogeneous polynomial of degree four in $q$ and $p$. Next we impose this expression to be zero, resulting in 28 conditions that the coefficients $a_{i}$ and $b_{j}$ have to verify. All these equations are linear in $b_{i}$, quadratic in $a_{j}$ and homogeneous. Thus, we can treat them as an overdetermined linear system with 28 equations in the 15 unknowns $b_{i}$ whereas the quadratic terms in the $a_{i}$ are treated as the independent terms. One checks that the rank of the (numeric) matrix involving the coefficients of the $b_{i}$ is 12 whereas the rank of the augmented matrix involving the independent terms can be 12 or 13 . Applying Gaussian elimination we put the system in row echelon form concluding that the rank of the augmented matrix is 12 if and only if three relations among the $a_{i}$ hold. In addition, one can put twelve of the fifteen $b_{i}$ in terms of the $a_{j}$ and the three remaining $b_{k}$. The resulting conditions appear in Table 4 . 


$$
\begin{aligned}
& b_{1}=\frac{1}{18}\left(45 a_{1}^{2}+5 a_{2}^{2}-5 a_{3}^{2}-45 a_{4}^{2}+5 a_{5}^{2}-5 a_{7}^{2}+18 b_{5}\right), \\
& b_{2}=\frac{5}{9}\left(6 a_{1} a_{2}+2 a_{2} a_{3}+a_{5} a_{6}\right) \\
& b_{3}=\frac{1}{18}\left(30 a_{1} a_{3}+20 a_{2}^{2}+30 a_{2} a_{4}+10 a_{3}^{2}-90 a_{4}^{2}+10 a_{5} a_{7}+5 a_{6}^{2}-10 a_{7}^{2}+36 b_{5}\right), \\
& b_{4}=\frac{5}{9}\left(2 a_{2} a_{3}+6 a_{3} a_{4}+a_{6} a_{7}\right), \\
& b_{6}=\frac{5}{9}\left(6 a_{1} a_{5}+a_{2} a_{6}+2 a_{5} a_{8}\right) \\
& b_{7}=\frac{5}{3}\left(2 a_{2} a_{5}+a_{3} a_{6}+a_{6} a_{8}\right) \\
& b_{8}=\frac{5}{3}\left(2 a_{3} a_{5}+3 a_{4} a_{6}+2 a_{7} a_{8}\right), \\
& b_{9}=\frac{5}{9}\left(a_{3} a_{6}+6 a_{4} a_{7}+2 a_{7} a_{9}\right) \\
& b_{11}=\frac{5}{3}\left(2 a_{2} a_{8}+2 a_{3} a_{9}+3 a_{6} a_{10}\right) \\
& b_{12}=\frac{1}{3}\left(-5 a_{5}^{2}+5 a_{7}^{2}-5 a_{8}^{2}+5 a_{9}^{2}+3 b_{10}\right) \\
& b_{13}=\frac{5}{9}\left(2 a_{5} a_{8}+a_{6} a_{9}+6 a_{8} a_{10}\right), \\
& b_{14}=\frac{5}{9}\left(a_{6} a_{8}+2 a_{7} a_{9}+6 a_{9} a_{10}\right), \\
& 3 a_{1} a_{6}-2 a_{2} a_{5}+2 a_{2} a_{7}-a_{3} a_{6}+2 a_{5} a_{9}-a_{6} a_{8}=0 \\
& a_{2} a_{6}-2 a_{3} a_{5}+2 a_{3} a_{7}-3 a_{4} a_{6}-2 a_{7} a_{8}+a_{6} a_{9}=0 \\
& 2 a_{2} a_{8}+2 a_{3} a_{9}-a_{5} a_{6}-a_{6} a_{7}+3 a_{6} a_{10}-2 a_{8} a_{9}=0 .
\end{aligned}
$$

TABLE 4 . The relations among the parameters $a_{j}, b_{j}$ to obtain the normal form with axial symmetry. There are fifteen independent constraints out of the 25 coefficients.

Table 3 of reference [34] also provides conditions to get a normal form Hamiltonian which is axially symmetric up to terms of degree four in $q$ and $p$. However, these conditions that were obtained using nodal-Lissajous coordinates are a bit more involved than the ones of Table 4 .

The twice reduced Hamiltonian $\mathcal{K}_{\epsilon}$ is obtained as follows. First, we reduce the normal form Hamiltonian by the oscillator symmetry using the method described in Section 4, that is, performing the division algorithm for multivariate polynomials with respect to the Gröbner basis of the invariants $\rho_{i}$ together with their relations, arriving at a Hamiltonian written exclusively in terms of the $\rho_{i}$. This step is achieved for arbitrary $a_{i}, b_{j}$. The second reduction process can be carried out only when the combinations of the parameters appearing in Table 4 hold. From this table, it is readily deduced that the $b_{i}$ can be put in terms of the $a_{j}$ and $b_{5}$, $b_{10}$ whereas $b_{15}$ is free. The three equations involving the $a_{i}$ but not the $b_{j}$ are 
now solved, getting six solutions. This leads to six different possibilities for the Hamiltonian function in $\mathbb{C P}_{h}^{2}$. For each of the six possible reduced Hamiltonians we apply the method of Section 5 to reduce them by the axial symmetry, ending up with six Hamiltonians written always in terms of $\tau_{1}, \tau_{2}$ and $\tau_{2}^{2}$ with coefficients $\alpha^{*}, \beta^{*}$ and $\delta^{*}$ depending on the parameters $a_{i}, b_{j}, L$ and $N$.

The results on the equilibrium points, stability and bifurcations obtained in Section 5 apply now to Hamiltonian (83) if one replaces $\alpha, \beta$ and $\delta$ by $\alpha^{*}, \beta^{*}$ and $\delta^{*}$ for the six possible situations. Thus, one obtains information of the dynamics of Hamiltonian (6) with the perturbation given in (4) and the coefficients satisfying the conditions of Table 4 using the information on the dynamics from the twice reduced Hamiltonian $\mathcal{K}_{\epsilon}$. Two exceptions to this are the degeneracies observed in (60) when $\tilde{\alpha}=\beta=\tilde{\delta}=0$ and the degenerate Hamiltonian Hopf bifurcations of Theorem 5.5 since then higher order terms of the normal form are used. In this case one should carry out the calculations of this section to order four in $\epsilon$, imposing more conditions on the coefficients $a_{i}, b_{j}$ to get axially symmetric normal forms with the aim of resolving the existing degeneracies.

To achieve the proof of (ii), we select all possible equilibria in $\mathcal{T}_{L, N}$ that are elliptic points (non-degenerate centres) and apply the results of Subsection 6.1 where instead of considering the parameter $\alpha, \beta$ and $\delta$ one uses $\alpha^{*}, \beta^{*}$ and $\delta^{*}$ for the six different versions of them.

Remark 11. The previous theorem is also satisfied if the conditions of Table 4 are only approximately true. Notice that in this case one can arrange the Hamiltonian so that up to terms of order $\epsilon^{2}$ it is axially-symmetric while the higher-order terms break this symmetry. Then the class of Hamiltonians that satisfies the previous theorem concerns a large open subset of the parameters' set.

Remark 12. Apparently, the restrictions given in Table 4 are not equivalent to those appearing in [34]. It is not easy to establish a connection between them. 
Perhaps one could build a Gröbner basis trying to reduce the conditions of Table 3 in [34] to the ones we have obtained here but it is not immediate and is out of the scope of this paper.

\section{Conclusions}

In this paper we generalise the work on the 1:1:1 resonance initiated in [16] and [31] applying singular reduction theory and the invariants of the different reductions. Preliminary studies indicate that the results obtained here can be extended to the 1:-1:1 resonance [66].

We perform a detailed analysis in three different reduced spaces, reconstructing the dynamics of the full problem in the three cases. In this respect our study presents novelties compared to previous approaches on the same topic, both regarding the conclusions on the dynamics of the full system and the methodology applied.

We introduce two sets of rectangular symplectic coordinates in $\mathbb{C P}_{h}^{2}$ that allow us to make a local study of the relative equilibria straightforwardly, shortening the treatments of previous works. Similarly we have defined new sets of symplectic coordinates in $\mathcal{M}_{\gamma}$ and in $\mathcal{T}_{L, N}$. In general, the use of these variables simplifies considerably previous approaches whose purposes were the analysis of the neighbourhoods of different types of relative equilibria.

The study of the flow in $\mathcal{T}_{L, N}$ involves three parameters and this leads to a reduced system with a rich dynamics where the parametric bifurcations are twodimensional surfaces. The reconstruction of the dynamics from the twice-reduced space accounts for the existence of invariant 2-tori and KAM 3-tori for the full Hamiltonian. Besides, the parametric bifurcations associated to the relative equilibria translate to bifurcations of 2-tori in $T^{*} \mathbb{R}^{3}$.

The analysis carried out in Section 5 can be applied to systems that are not axially-symmetric but whose second order normal form in $\epsilon$ enjoys this symmetry. For these Hamiltonians the existence of the KAM tori that has been established for 
the axially-symmetric Hamiltonians remains valid, enlarging therefore the KAMtype analysis performed in the paper to a large class of Hamiltonians enjoying the 1:1:1 resonance.

Hamiltonian Hopf bifurcations have been determined in Section 5. The degenerate cases of this bifurcation are related to the integrability of some families of potentials in 1:1:1 resonance. In this respect we have found a family of systems that could be integrable. This family contains the integrable case studied in [31].

The application of the KAM theorem by Han, Li and Yi is crucial to prove the existence of the different types of KAM 3-tori due to the high degeneracy of the Hamiltonian system. Moreover, an interesting issue to deal with in future is the proof of the persistence of lower dimensional tori in this context of high degeneracy.

Finally, the use of tools from computer algebra, such as the Gröbner bases and the division algorithm for multivariate polynomials, has been essential in order to handle the large symbolic expressions appearing in the computations.

\section{Acknowledgments}

We appreciate the valuable remarks made by the anonymous referees.

\section{REFERENCES}

[1] E. VAN DER AA, First-order resonances in three-degrees-of-freedom systems, Celestial Mech. 31 (2) (1983), 163-191.

[2] J. M. Arms, R. H. Cushman, And M. J. Gotay, A universal reduction procedure for Hamiltonian group actions, in The Geometry of Hamiltonian Systems, T. Ratiu ed., SpringerVerlag, New York, 1991, pp. 33-51.

[3] V. I. Arnold, V. V. Kozlov, and A. I. Neishtadt, Mathematical Aspects of Classical and Celestial Mechanics, Third Edition, Encyclopaedia of Mathematical Sciences, Vol. 3. Dynamical Systems III, Springer-Verlag, New York, 2006.

[4] S. Breiter, A. Elipe, and I. Wytrzyszczak, Analytical investigation of the orbital structure close to the 1:1:1 resonance in spheroidal galaxies, Astron. Astrophys. 431 (3) (2005), $1145-1155$. 
[5] H. W. Broer, H. Hanssmann, and J. Hoo, The quasi-periodic Hamiltonian Hopf bifurcation, Nonlinearity 20 (2) (2007) 417-460.

[6] H. W. Broen, G. B. Huitema, and M. B. Sevryuk, Quasi-Periodic Motions in Families of Dynamical Systems. Order amidst Chaos, Lecture Notes in Math. 1645, Springer-Verlag, New York, 1996.

[7] M. T. de Bustos, J. L. Guirao, J. Llibre, and J. Vera, New families of periodic orbits for a galactic potential, Chaos Solitons Fractals. 82 (2016), 97-102.

[8] N. D. Caranicolas, 1:1:1 resonant periodic orbits in 3-dimensional galactic-type Hamiltonians, Astron. Astrophys. 114 (1) (1982), 360-366.

[9] N. D. Caranicolas, and E. E. Zotos, Investigating the nature of motion in 3D perturbed elliptic oscillators displaying exact periodic orbits, Nonlinear Dyn. 69 (4) (2012) 1795-1805.

[10] J. F. Cariñena, A. Ibort, G. Marmo, and G. Morandi, Geometry from Dynamics, Classical and Quantum, Springer-Verlag, Dordrecht, 2015.

[11] R. C. Churchill, M. Kummer, And D. L. Rod, On averaging, reduction, and symmetry in Hamiltonian systems, J. Differential Equations. 49 (3) (1983), 359-414.

[12] G. Contopoulos, And B. Barbanis, Resonant systems with three degrees of freedom, Astron. Astrophys. 153 (1) (1985), 44-54.

[13] O. Cornea, G. Lupton, J. Oprea, and D. Tanré, Lusternik-Schnirelmann Category, Mathematical Surveys and Monographs 103, American Mathematical Society, Rhode Island, 2003.

[14] D. Cox, J. Little, And D. O’Shea, Ideals, Varieties, and Algorithms. An Introduction to Computational Algebraic Geometry and Commutative Algebra, third edition, Springer-Verlag, New York, 2007.

[15] R. H. Cushman, And L. M. Bates, Global Aspects of Classical Integrable Systems, second edition, Birkhäuser Verlag, Basel, 2015.

[16] R. H. Cushman, S. Ferrer, And H. Hanssmann, Singular reduction of axially symmetric perturbations of the isotropic harmonic oscillator, Nonlinearity 12 (2) (1999), 389-410.

[17] D. David, D. D. Holm, And M. V. Tratnik, Hamiltonian chaos in nonlinear optical polarization dynamics, Physics Reports 187 (1990), 281-367.

[18] A. Deprit, Canonical transformations depending on a small parameter, Celestial Mech. 1 (1) (1969), 12-30.

[19] A. Deprit, The Lissajous transformation I. Basics, Celestial Mech. 51 (3) (1991), 201-225.

[20] A. Deprit, B. R. Miller, Normalization in the face of integrability, Ann. New York Acad. Sci. 536 (1988), 101-126. 
[21] K. Efstathiou, Metamorphoses of Hamiltonian Systems with Symmetries, Lecture Notes in Math. 1865, Springer-Verlag, New York, 2005.

[22] K. Efstathiou, and D. A. Sadovskí, Perturbations of the 1:1:1 resonance with tetrahedral symmetry: a three degree of freedom analogue of the two degree of freedom Hénon-Heiles Hamiltonian, Nonlinearity 17 (2) (2004), 415-446.

[23] K. Efstathiou, D. A. Sadovskí́, and R. H. Cushman, Linear Hamiltonian Hopf bifurcation for point-group-invariant perturbations of the 1:1:1 resonance, Proc. R. Soc. Lond. A 459 (2040) (2003), 2997-3019.

[24] K. Efstathiou, D. A. Sadovskí́, And B. I. Zhilinskí́, Analysis of rotation-vibration relative equilibria on the example of a tetrahedral four atom molecule, SIAM J. Appl. Dyn. Syst. 3 (3) (2004), 261-351.

[25] J. Egea, S. Ferrer, And J.-C. VAn Der Meer, Hamiltonian fourfold 1:1 resonance with two rotational symmetries, Regul. Chaotic Dyn. 12 (6) (2007), 664-674.

[26] J. Egea, S. Ferrer, And J.-C. van Der Meer, Bifurcations of the Hamiltonian fourfold 1:1 resonance with toroidal symmetry, J. Nonlinear Sci. 21 (6) (2011), 835-874.

[27] A. Elipe, Extended Lissajous variables for oscillators in resonance, Math. Comput. Simulation 57 (3-5) (2001), 217-226.

[28] D. Farrelly, J. Humpherys, and T. Uzer, Normalization of resonant Hamiltonians, in Hamiltonian Mechanics, J. Seimenis, ed., Plenum Press, New York, 1994, pp. 237-244.

[29] D. Farrelly, and T. Uzer, Normalization and the detection of integrability: The generalized van der Waals potential, Celestial Mech. Dynam. Astronom. 61 (1) (1995), 71-95.

[30] S. Ferrer, and J. Gárate, On perturbed 3D elliptic oscillators: A case of critical inclination in galactic dynamics, in New Trends for Hamiltonian Systems and Celestial Mechanics, E. A. Lacomba and J. Llibre, ed., Cocoyoc 1994, Advanced Series in Nonlinear Dynamics, Vol. 8, World Scientific, Singapore, 1996, pp. 179-97.

[31] S. Ferrer, H. Hanssmann, J. F. Palacián, and P. Yanguas, On perturbed oscillators in 1-1-1 resonance: the case of axially symmetric cubic potentials, J. Geom. Phys. 40 (3-4) (2002), 320-369.

[32] S. Ferrer, M. Lara, J. F. Palacián, J. F. San Juan, A. Viartola, and P. Yanguas, The Hénon and Heiles problem in three dimensions. I. Periodic orbits near the origin, Internat. J. Bifur. Chaos Appl. Sci. Engrg. 8 (6) (1998), 1199-1213.

[33] S. Ferrer, M. Lara, J. F. Palacián, J. F. San Juan, A. Viartola, and P. Yanguas, The Hénon and Heiles problem in three dimensions. II. Relative equilibria and bifurcations in the reduced system, Internat. J. Bifur. Chaos Appl. Sci. Engrg. 8 (6) (1998), 1215-1229. 
[34] S. Ferrer, J. F. Palacián, and P. Yanguas, Hamiltonian oscillators in 1-1-1 resonance: normalization and integrability, J. Nonlinear Sci. 10 (2) (2000), 145-174.

[35] J. L. G. Guirao, J. Llibre, And J. A. Vera, Periodic orbits of a perturbed 3-dimensional isotropic oscillator with axial symmetry, Nonlinear Dyn. 83 (2016), 839-848.

[36] M. C. Gutzwiller, Chaos in Classical and Quantum Mechanics, Interdisciplinary Applied Mathematics 1, Springer-Verlag, New York, 1990.

[37] G. Haller, Chaos Near Resonance, Applied Mathematical Sciences 138, Springer-Verlag, New York, 1999.

[38] G. Haller, And S. Wiggins, Geometry and chaos near resonant equilibria of 3-DOF Hamiltonian systems, Phys. D 90 (4) (1996), 319-365.

[39] Y. HAN, Y. LI, AND Y. YI, Invariant tori in Hamiltonian systems with high order proper degeneracy, Ann. Henri Poincaré 10 (8) (2010), 1419-1436.

[40] H. Hanssmann, Local and Semi-Local Bifurcations in Hamiltonian Dynamical Systems: Results and Examples, Lecture Notes in Math. 1893, Springer-Verlag, New York, 2007.

[41] H. Hanssmann, And J.-C. VAn Der Meer, On the Hamiltonian Hopf bifurcations in the 3D Hénon-Heiles family, J. Dynam. Differential Equations 14 (3) (2002), 675-695.

[42] H. Hanssmann, And J.-C. VAn Der MeEr, Algebraic methods for determining Hamiltonian Hopf bifurcations in three-degree-of-freedom systems, J. Dynam. Differential Equations 17 (3) (2005), 453-474.

[43] M. Kummer, On resonant classical Hamiltonians with $n$ frequencies, J. Differential Equations 83 (2) (1990), 220-243.

[44] V. Lanchares, J. F. Palacián, A. I. Pascual, J. P. Salas, and P. Yanguas, Perturbed ion traps: A generalization of the three-dimensional Hénon-Heiles problem, Chaos 12 (1) (2002), 87-99.

[45] A. J. Laub, And K. R. Meyer, Canonical forms for symplectic and Hamiltonian matrices, Celes. Mech. 9 (2) (1974), 213-238.

[46] F. E. Lembarki, And J. Llibre, Periodic orbits for the generalized Yang-Mills Hamiltonian system in dimension 6, Nonlinear Dynam. 76 (3) (2014), 1807-1819.

[47] A. J. Lichtenberg, and M. A. Lieberman, Regular and Chaotic Dynamics, second edition, Applied Mathematical Sciences 38, Springer-Verlag, New York, 1992.

[48] J. Llibre, D. Pasca, And C. Valls, Periodic solutions of a galactic potential, Chaos Solitons Fractals 61 (2014), 38-43.

[49] J. LliBRE, AND C. VIDAL, New periodic solutions in 3-dimensional galactic-type Hamiltonian systems, Nonlinear Dynam. 78 (2) (2014), 969-980. 
[50] A. P. Markeev, Libration Points in Celestial Mechanics and Space Dynamics, Nauka, Moscow, 1978.

[51] J. Marsden, And A. Weinstein, Reduction of symplectic manifolds with symmetry, Rep. Math. Phys. 5 (1) (1974), 121-130.

[52] J.-C. VAn Der Meer, The Hamiltonian Hopf Bifurcation, Lecture Notes in Math. 1160, Springer-Verlag, New York, 1985.

[53] K. R. Meyer, Symmetries and integrals in mechanics, in Dynamical Systems, M. M. Peixoto, ed., Academic Press, New York, 1973, pp. 259-272.

[54] K. R. Meyer, G. R. Hall, And D. Offin, Introduction to Hamiltonian Dynamical Systems and the $N$-Body Problem, second edition, Springer-Verlag, New York, 2009.

[55] K. R. Meyer, J. F. Palacián, and P. Yanguas, Geometric averaging of Hamiltonian systems: periodic solutions, stability, and KAM tori, SIAM J. Appl. Dyn. Syst. 10 (3) (2011), 817-856.

[56] K. R. Meyer, J. F. Palacián, and P. Yanguas, Invariant tori in the Lunar problem, Publ. Mat. Extra (2014), 353-394.

[57] K. R. Meyer, J. F. Palacián, and P. Yanguas, Singular reduction of resonant Hamiltonians, submitted (2016).

[58] K. R. Meyer, J. F. Palacián, and P. Yanguas, Singular reduction of high dimensional Hamiltonian systems, in preparation.

[59] K. R. Meyer, And D. S. Schmidt, Periodic orbits near $L_{4}$ for mass ratios near the critical mass ratio of Routh, Celestial Mech. 4 (1) (1971), 99-109.

[60] J. Moser, Regularization of Kepler's problem and the averaging method on a manifold, Comm. Pure Appl. Math. XXIII (4) (1970), 609-636.

[61] J. F. Palacián, C. Vidal, J. Vidarte, and P. Yanguas, Periodic solutions and KAM tori in a triaxial potential, to be published in SIAM J. Appl. Dyn. Syst. (2017).

[62] G. REeB, Sur certaines propriétés topologiques des trajectoires des systèmes dynamiques, Acad. Roy. Sci. Lett. et Beaux-Arts de Belgique. Cl. des Sci. Mém. in 8 , Ser. 2, 27 (1952), No. 9.

[63] J. A. SAnders, Normal forms of 3 degree of freedom Hamiltonian systems at equilibrium in the resonant case, in Global Integrability of Field Theories, J. Calmet, W. M. Seiler and R. W. Tucker, eds., Univ. Karlsruhe, Karlsruhe, 2006, pp. 335-346.

[64] H. Schomerus, Periodic orbits near bifurcations of codimension two: Classical mechanics, semiclassics and Stokes transitions, J. Phys. A: Math. Gen. 31 (18) (1998), 4167-4196.

[65] B. Sturmfels, Algorithms in Invariant Theory, Texts and Monographs in Symbolic Computation, Springer-Verlag, New York, 1993. 
[66] J. Vidarte, Averaging, Reduction and Reconstruction in Hamiltonian Systems and Applications to Problems of Celestial Mechanics, Ph.D. Thesis, Universidad del Bío-Bío, 2017.

[67] A. Weinstein, Symplectic V-manifolds, periodic orbits of Hamiltonian systems, and the volume of certain Riemannian manifolds, Comm. Pure Appl. Math. XXX (2) (1977), 265271.

[68] A. Weinstein, Bifurcations and Hamilton's principle, Math. Z. 159 (3) (1978), 235-248.

[69] T. Welker, The Bifurcation Diagram of the Second Nontrivial Normal Form of an Axially Symmetric Perturbation of the Isotropic Harmonic Oscillator, Bachelor Thesis, University of Utrecht, 2014.

[70] P. Yanguas, Integrability, Normalization and Symmetries of Hamiltonian Systems in 1-1-1 resonance, Ph.D. Thesis, Universidad Pública de Navarra, 1998.

[71] P. Yanguas, J. F. Palacián, K. R. Meyer, and H. S. Dumas, Periodic solutions in Hamiltonian systems, averaging, and the Lunar problem, SIAM J. Appl. Dyn. Syst. 7 (2) (2011), $311-340$.

[72] T. DE Zeeuw, Motion in the core of a triaxial potential, Mon. Not. R. Astr. Soc. 215 (1985), $731-760$.

[73] T. DE ZeEuw, Dynamical models for axisymmetric and triaxial galaxies, in Structure and Dynamics of Elliptical Galaxies, T. de Zeeuw, ed., IAU Symp., Vol. 127, Reidel, Dordrecht, 1987, pp. 271-290.

[74] T. de Zeeuw, And M. Franx, Structure and dynamics of elliptical galaxies, Annu. Rev. Astron. Astrophys. 29 (1991) 239-274.

[75] T. De Zeeuw, And D. Merritt, Stellar orbits in a triaxial galaxy. I. Orbits in the plane of rotation, Astrophys. J. 267 (1983), 571-595. 
(D. Carrasco) Departamento de Matemática, Facultad de Ciencias, Universidad del Bío-Bío, Concepción, ViII Región, Chile

E-mail address: dcarrasc@ubiobio.cl

(J.F. Palacián) Departamento de Ingeniería Matemática e Informática and Institute for Advanced Materials (INAMAT), Universidad Pública de Navarra, Campus de ArroSAdia, 31006 PAmplona, Spain

E-mail address: palacian@unavarra.es

(C. Vidal) Departamento de Matemática, Facultad de Ciencias, Universidad del BíoBío, Concepción, Viit Región, Chile

E-mail address: clvidal@ubiobio.cl

(J. Vidarte) Departamento de Matemática, Facultad de Ciencias, Universidad del BíoBío, Casilla 5-C, Concepción, Viit Región, Chile

E-mail address: jvidarte@ubiobio.cl

(P. Yanguas) Departamento de Ingeniería Matemática e Informática and Institute for Advanced Materials (InAmat), Universidad Pública de Navarra, Campus de Arrosadia, 31006 Pamplona, Spain

E-mail address: yanguas@unavarra.es 